Supporting Materials for

\title{
Evaluation of Bis(phosphine) Ligands for Ethylene Oligomerization: Discovery of Alkyl Phosphines as Effective Ligands for Ethylene Tri- and Tetramerization.
}

Scott D. Boelter, Dan R. Davies, Kara A. Milbrandt, David R. Wilson, Molly Wiltzius, Mari S. Rosen* and Jerzy Klosin*

Corporate R\&D, The Dow Chemical Company, 1776 Building, Midland, MI 48667

E-mail: msrosen@dow.com (MSR), jklosin@dow.com (JK) 


\section{TABLE OF CONTENTS}

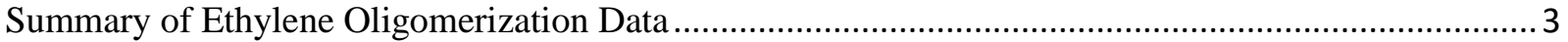

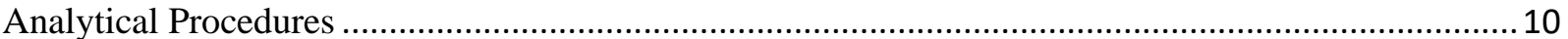

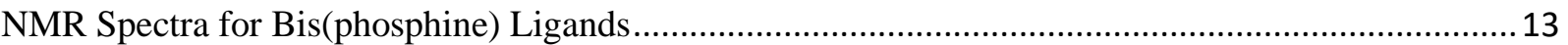

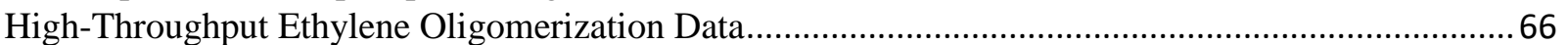

Example of Activity and Selectivity Calculations: Ligand 1 and Ligand 27 .......................................76 
Summary of Ethylene Oligomerization Data

Table S1. Ligands Evaluated for Ethylene Oligomerization with $\mathrm{CrCl}_{3}(\mathrm{THF})_{3}$ in a High-Throughput Reactor

\begin{tabular}{|c|c|c|c|c|c|c|c|c|}
\hline \multirow{2}{*}{$\begin{array}{l}\text { Ligand } \\
\quad \#\end{array}$} & \multirow{2}{*}{ Ligand } & \multirow{2}{*}{$\begin{array}{l}\text { Product } \\
\text { yield } \\
(\mathrm{mg})\end{array}$} & \multirow{2}{*}{$\begin{array}{l}\text { Activity }{ }^{\mathrm{a}, \mathrm{b}} \\
\text { (g product/ } \\
\mathrm{g} \mathrm{Cr} \mathrm{hr} \text { ) }\end{array}$} & \multicolumn{5}{|c|}{ Selectivity $^{\mathrm{a}, \mathrm{b}}(\mathrm{wt} \%)$} \\
\hline & & & & 1-Octene & 1-Hexene & Cyclics & $\mathrm{C} 10-\mathrm{C} 18$ & HDPE \\
\hline 1 & & 639.4 & 492,000 & 62.0 & 24.9 & 4.2 & 6.7 & 2.2 \\
\hline 2 & & 206.7 & 159,000 & 53.7 & 23.5 & 8.5 & 5.6 & 8.6 \\
\hline 3 & & 726.7 & 559,000 & 52.4 & 35.2 & 4.0 & 5.2 & 3.2 \\
\hline 4 & & 274.5 & 211,000 & 45.4 & 13.0 & 19.2 & 11.1 & 11.0 \\
\hline 6 & & 590.1 & 454,000 & 55.6 & 22.3 & 11.6 & 5.0 & 5.5 \\
\hline 10 & & 270.8 & 978,000 & 4.7 & 90.4 & 0.1 & 4.2 & 0.6 \\
\hline 11 & & 688.6 & 529,000 & 0.4 & 97.2 & 0.0 & 1.6 & 0.8 \\
\hline 12 & & 78.4 & 60,000 & 8.0 & 52.7 & 1.3 & 2.7 & 35.3 \\
\hline 13 & & 8.6 & 7,000 & 2.0 & 2.5 & 0.8 & 2.1 & 92.4 \\
\hline 14 & & 144.4 & 111,000 & 0.3 & 16.3 & 0.1 & 2.3 & 81.1 \\
\hline
\end{tabular}




\begin{tabular}{|c|c|c|c|c|c|c|c|c|}
\hline \multirow{2}{*}{$\begin{array}{l}\text { Ligand } \\
\#\end{array}$} & \multirow{2}{*}{ Ligand } & \multirow{2}{*}{$\begin{array}{c}\text { Product } \\
\text { yield }^{\mathrm{a}, \mathrm{b}}(\mathrm{mg})\end{array}$} & \multirow{2}{*}{$\begin{array}{c}\text { Activity }{ }^{\mathrm{a}, \mathrm{b}} \\
\text { (g product/ } \\
\text { g Cr hr) }\end{array}$} & \multicolumn{5}{|c|}{ Selectivity ${ }^{\mathrm{a}, \mathrm{b}}(\mathrm{wt} \%)$} \\
\hline & & & & 1-Octene & 1-Hexene & Cyclics & $\mathrm{C} 10-\mathrm{C} 18$ & HDPE \\
\hline 15 & & 144.3 & 111,000 & 7.1 & 12.3 & 3.4 & 30.9 & 46.2 \\
\hline 16 & & 204.7 & 157,000 & 1.2 & 0.2 & 0.0 & 0.7 & 97.8 \\
\hline 17 & & 60.3 & 46,000 & 9.9 & 67.6 & 2.2 & 3.9 & 16.5 \\
\hline 18 & & 8.8 & 6,000 & 5.1 & 11.8 & 4.2 & 9.9 & 69.0 \\
\hline 19 & & 8.5 & 6,000 & 1.3 & 1.3 & 1.6 & 1.5 & 94.3 \\
\hline 20 & & 150.3 & 114,000 & 41.5 & 12.7 & 27.7 & 10.5 & 7.1 \\
\hline 21 & & 28.2 & 22,000 & 3.2 & 67.2 & 1.2 & 3.8 & 24.6 \\
\hline 22 & & 79.2 & 61,000 & 35.7 & 12.9 & 27.0 & 14.4 & 10.1 \\
\hline 23 & & 453.2 & 349,000 & 33.9 & 48.1 & 9.3 & 7.2 & 1.5 \\
\hline 24 & & 10.7 & 8,000 & 0.0 & 26.8 & 1.9 & 3.4 & 67.9 \\
\hline
\end{tabular}




\begin{tabular}{|c|c|c|c|c|c|c|c|c|}
\hline \multirow{2}{*}{$\begin{array}{c}\text { Ligand } \\
\#\end{array}$} & \multirow{2}{*}{ Ligand } & \multirow{2}{*}{$\begin{array}{l}\text { Product } \\
\text { yieldd, } \\
(\mathrm{mg})\end{array}$} & \multirow{2}{*}{$\begin{array}{l}\text { Activity }{ }^{\mathrm{a}, \mathrm{b}} \\
\text { (g product/ } \\
\text { g Cr hr) }\end{array}$} & \multicolumn{5}{|c|}{ Selectivity ${ }^{\mathrm{a}, \mathrm{b}}(\mathrm{wt} \%)$} \\
\hline & & & & 1-Octene & 1-Hexene & Cyclics & $\mathrm{C} 10-\mathrm{C} 18$ & HDPE \\
\hline 25 & & 40.0 & 31,000 & 0.0 & 78.3 & 0.3 & 2.4 & 19.0 \\
\hline 26 & & 116.6 & 90,000 & 1.2 & 79.6 & 0.8 & 3.6 & 14.8 \\
\hline 27 & & 240.2 & 185,000 & 50.0 & 31.8 & 12.6 & 2.1 & 3.5 \\
\hline 28 & & 60.5 & 46,000 & 37.3 & 6.7 & 11.1 & 21.1 & 23.7 \\
\hline 29 & & 39.4 & 30,000 & 2.8 & 5.8 & 0.3 & 3.3 & 87.7 \\
\hline 30 & & 21.2 & 16,000 & 21.6 & 11.1 & 5.2 & 11.0 & 51.1 \\
\hline 31 & & 282.2 & 217,000 & 52.2 & 26.6 & 8.2 & 5.2 & 7.8 \\
\hline 32 & & 668.4 & 514,000 & 53.4 & 35.0 & 4.1 & 5.1 & 2.3 \\
\hline 33 & & 78.1 & 60,000 & 0.5 & 18.4 & 0.1 & 3.8 & 77.2 \\
\hline 34 & & 43.0 & 33,000 & 1.5 & 1.7 & 0.3 & 11.0 & 85.5 \\
\hline 35 & & 46.0 & 35,000 & 0.5 & 20.3 & 0.2 & 7.5 & 71.4 \\
\hline
\end{tabular}




\begin{tabular}{|c|c|c|c|c|c|c|c|c|}
\hline \multirow{2}{*}{$\begin{array}{l}\text { Ligand } \\
\quad \#\end{array}$} & \multirow{2}{*}{ Ligand } & \multirow{2}{*}{$\begin{array}{l}\text { Product } \\
\text { yield } \\
(\mathrm{mg})\end{array}$} & \multirow{2}{*}{$\begin{array}{l}\text { Activity }^{\mathrm{a}, \mathrm{b}} \\
\text { (g product/ } \\
\mathrm{g} \mathrm{Cr} \mathrm{hr} \text { ) }\end{array}$} & \multicolumn{5}{|c|}{ Selectivity $^{\mathrm{a}, \mathrm{b}}(\mathrm{wt} \%)$} \\
\hline & & & & 1-Octene & 1-Hexene & Cyclics & $\mathrm{C} 10-\mathrm{C} 18$ & HDPE \\
\hline 36 & & 25.3 & 19,000 & 0.7 & 11.6 & 0.2 & 5.3 & 82.2 \\
\hline 37 & & 8.0 & 6,000 & 2.3 & 5.4 & 1.3 & 5.8 & 85.1 \\
\hline 38 & & 11.8 & 9,000 & 2.3 & 2.5 & 0.9 & 5.4 & 88.9 \\
\hline 39 & & 16.3 & 13,000 & 2.2 & 2.8 & 1.9 & 15.5 & 77.6 \\
\hline 40 & & 18.0 & 14,000 & 0.5 & 1.1 & 1.7 & 1.5 & 96.2 \\
\hline 41 & & 80.8 & 62,000 & 27.1 & 6.5 & 5.3 & 11.8 & 49.3 \\
\hline 42 & & 93.4 & 72,000 & 0.3 & 0.7 & 0.1 & 2.5 & 96.4 \\
\hline
\end{tabular}




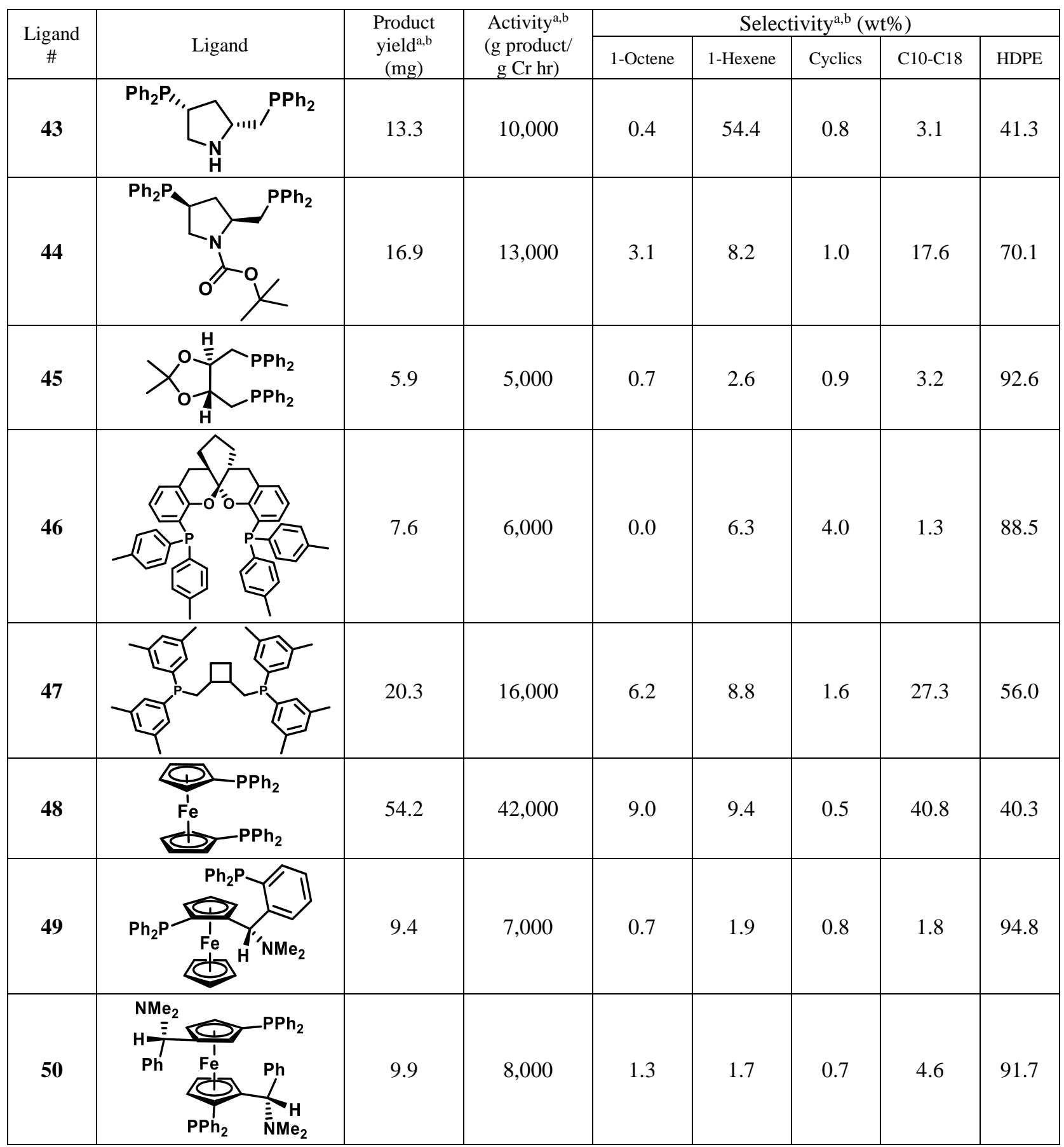




\begin{tabular}{|c|c|c|c|c|c|c|c|c|}
\hline \multirow{2}{*}{$\begin{array}{l}\text { Ligand } \\
\#\end{array}$} & \multirow{2}{*}{ Ligand } & \multirow{2}{*}{$\begin{array}{l}\text { Product } \\
\text { yieldd,b } \\
(\mathrm{mg})\end{array}$} & \multirow{2}{*}{$\begin{array}{l}\text { Activity }{ }^{\mathrm{a}, \mathrm{b}} \\
\text { (g product/ } \\
\text { g Cr hr) }\end{array}$} & \multicolumn{5}{|c|}{ Selectivity ${ }^{\mathrm{a}, \mathrm{b}}(\mathrm{wt} \%)$} \\
\hline & & & & 1-Octene & 1-Hexene & Cyclics & C10-C18 & HDPE \\
\hline 51 & & 17.2 & 13,000 & 5.6 & 14.5 & 0.9 & 27.4 & 51.7 \\
\hline 52 & & 8.2 & 6,000 & 1.4 & 9.2 & 17.7 & 3.2 & 68.5 \\
\hline 53 & & 10.4 & 8,000 & 3.1 & 10.6 & 8.0 & 8.1 & 70.3 \\
\hline 54 & & 5.7 & 4,000 & 0.9 & 1.0 & 1.6 & 1.2 & 95.3 \\
\hline 55 & & 8.3 & 6,000 & 0.5 & 1.6 & 2.7 & 5.4 & 89.9 \\
\hline 56 & & 6.8 & 5,000 & 0.0 & 2.7 & 0.0 & 5.6 & 91.7 \\
\hline 57 & & 28.0 & 22,000 & 0.0 & 67.3 & 0.4 & 2.6 & 29.8 \\
\hline
\end{tabular}

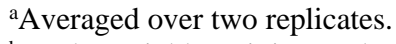

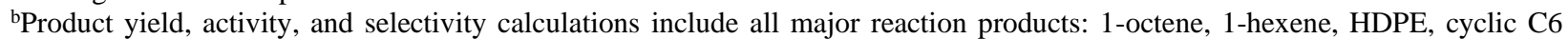
products (methylcyclopentane and methylenecyclopentane), and heavier $\alpha$-olefin (C10-C18) compounds. Sample calculations are provided on pg 76. 
Table S2. Summary of Conditions for Ethylene Oligomerization Experiments

\begin{tabular}{|c|c|c|c|}
\hline \multicolumn{2}{|c|}{ Parameter } & Value & Notes \\
\hline \multicolumn{2}{|c|}{ Cr precursor } & $\mathrm{CrCl}_{3}(\mathrm{THF})_{3}$ & \\
\hline \multirow{2}{*}{$\begin{array}{l}\text { Catalyst } \\
\text { loading }\end{array}$} & Cr precursor & $0.05 \mu \mathrm{mol}$ & \\
\hline & Ligand & $0.06 \mu \mathrm{mol}$ & 1.2 equiv ligand \\
\hline \multicolumn{2}{|c|}{ Ethylene pressure } & $500 \mathrm{psi}$ & \\
\hline \multicolumn{2}{|c|}{ Reaction temperature } & $60^{\circ} \mathrm{C}$ & \\
\hline \multicolumn{2}{|c|}{ Reaction time } & 30 minutes & \\
\hline \multicolumn{2}{|c|}{ Solvent } & Methylcyclohexane & \\
\hline \multicolumn{2}{|c|}{ Activator } & 1000 equiv MMAO-3A & Source: AkzoNobel \\
\hline \multicolumn{2}{|c|}{ GC internal standard } & $50 \mathrm{mg}$ nonane & \\
\hline
\end{tabular}




\section{Analytical Procedures}

The liquid reaction products were analyzed on an Agilent 7890 GC System. See Table S3 for GC Conditions.

Table S3. GC Conditions

\begin{tabular}{|l|l|}
\hline GC & Agilent 7890 Series \\
\hline Column & Agilent (DB-5MS), $30 \mathrm{~m} \times 32 \mu \mathrm{m}, 1 \mu \mathrm{m}$ film \\
\hline Oven & $70{ }^{\circ} \mathrm{C}$ for $8 \mathrm{~min}$, ramp $50{ }^{\circ} \mathrm{C} / \mathrm{min}$ to $300{ }^{\circ} \mathrm{C}$ (hold $3.4 \mathrm{~min}$ ), total time: $16 \mathrm{~min}$ \\
\hline Inlet & $300{ }^{\circ} \mathrm{C}$, Split, $30: 1 \mathrm{split}$ ratio \\
\hline Carrier Gas & Hydrogen, $1.33 \mathrm{~mL} / \mathrm{min}$ constant flow \\
\hline Flame Ionization Detector & $320^{\circ} \mathrm{C}, 45 \mathrm{~mL} / \mathrm{min} \mathrm{H}_{2}, 450 \mathrm{~mL} / \mathrm{min}$ air \\
\hline Injection Volume & $1 \mu \mathrm{L}$, chlorobenzene wash solvent \\
\hline
\end{tabular}

Samples for GC analysis were prepared by quenching $75 \mu \mathrm{L}$ of the reaction mixture with $25 \mu \mathrm{L}$ of methanol. The response factors were determined for 1-octene, 1-hexene, methylcyclopentane, and methylenecyclopentane via calibration using a standard solution with known concentrations. The response factors used for the $\mathrm{C} 10$ to $\mathrm{C} 18$ fractions were determined using the terminal olefin of the same carbon length (e.g. 1-decene for the $\mathrm{C} 10$ fraction). The concentrations of the reaction products were reported by the $\mathrm{GC}$ on a $\mathrm{g} / \mathrm{g}$ nonane basis because nonane was included as an internal standard at a known concentration in the reaction tubes $(50 \mathrm{mg}$ of nonane per $5 \mathrm{~mL}$ of reaction mixture). The amounts of the various reaction products produced were calculated relative to the amount of nonane added to the reaction mixture.

Table S4. Retention Times for C10-C18 Products

\begin{tabular}{|c|c|}
\hline Product & Retention time $(\mathrm{min})$ \\
\hline Decenes & $10.00-11.00$ \\
\hline Dodecenes & $11.30-11.80$ \\
\hline Tetradecenes & $11.81-12.70$ \\
\hline Hexadecenes & $13.00-13.50$ \\
\hline Octadecenes & $13.70-14.20$ \\
\hline
\end{tabular}


Figure S1. Example GC Chromatograms

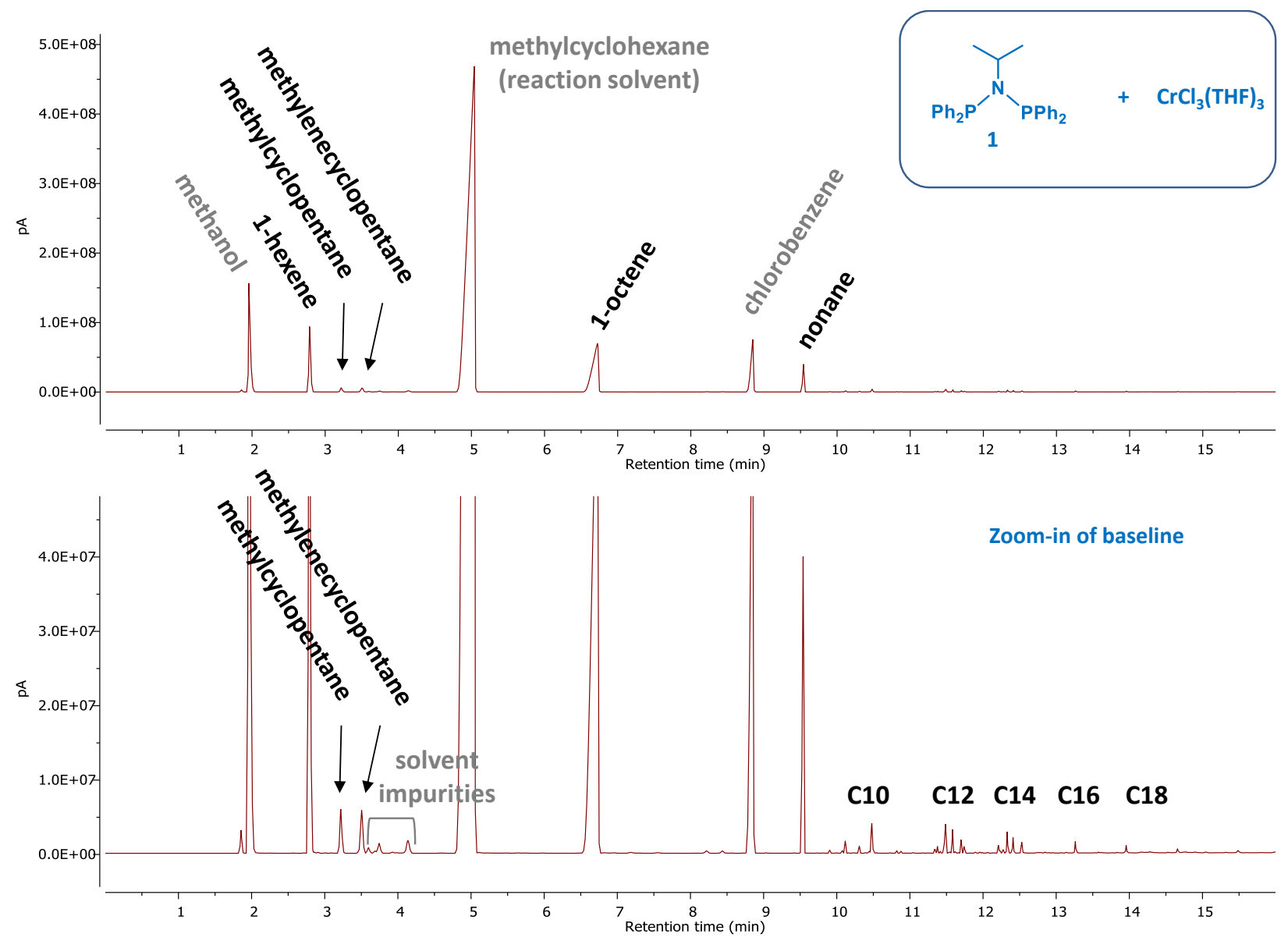




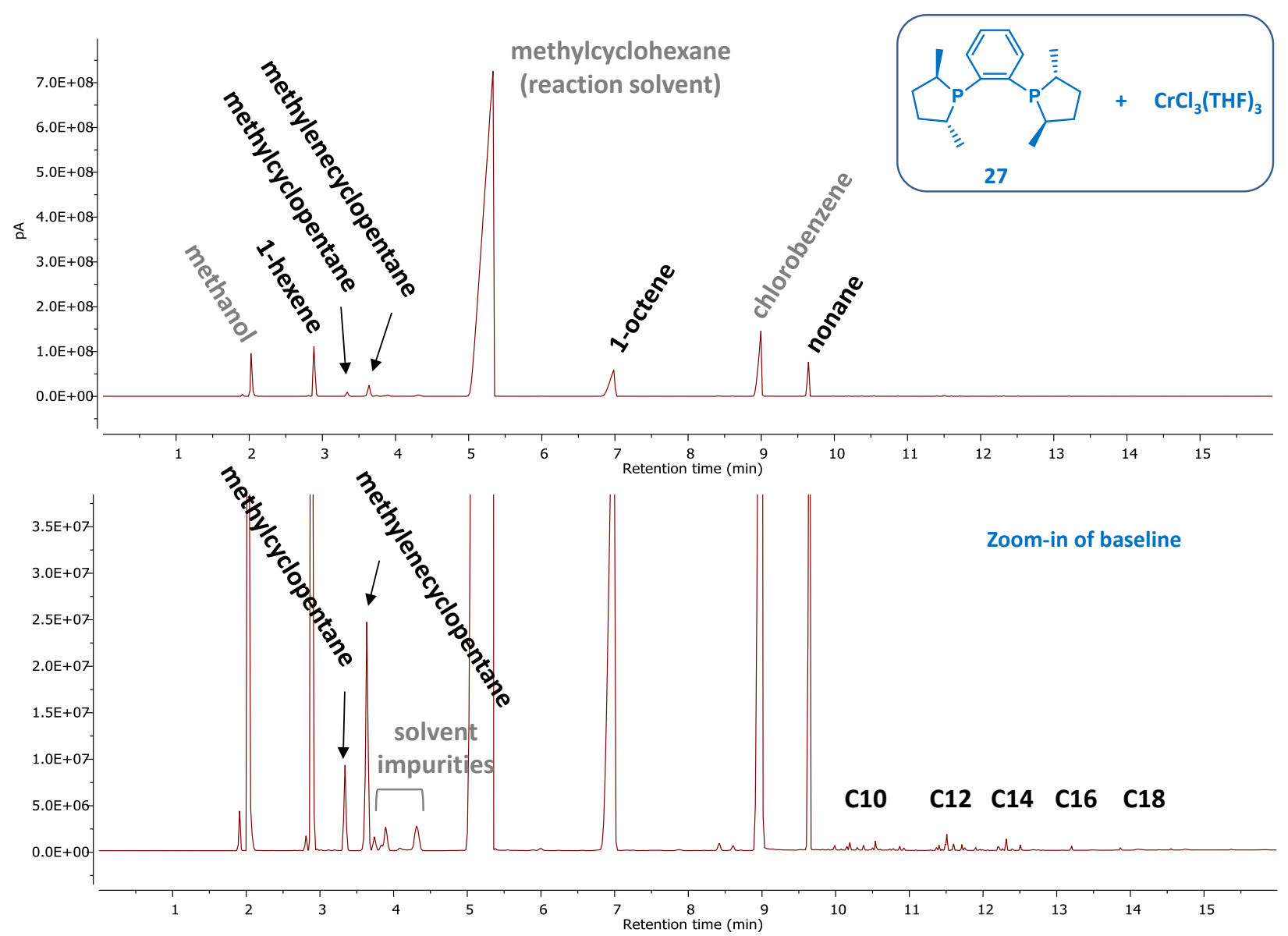




\section{NMR Spectra for Bis(phosphine) Ligands}

Figure S2. ${ }^{1} \mathrm{H}$ and ${ }^{31} \mathrm{P}$ NMR spectra of ligand 1 in $\mathrm{CDCl}_{3}$; $\boldsymbol{i}$-Pr-PNP, $N$-(diphenylphosphino)- $N$-(1methylethyl)- $P, P$-diphenylphosphinous amide.
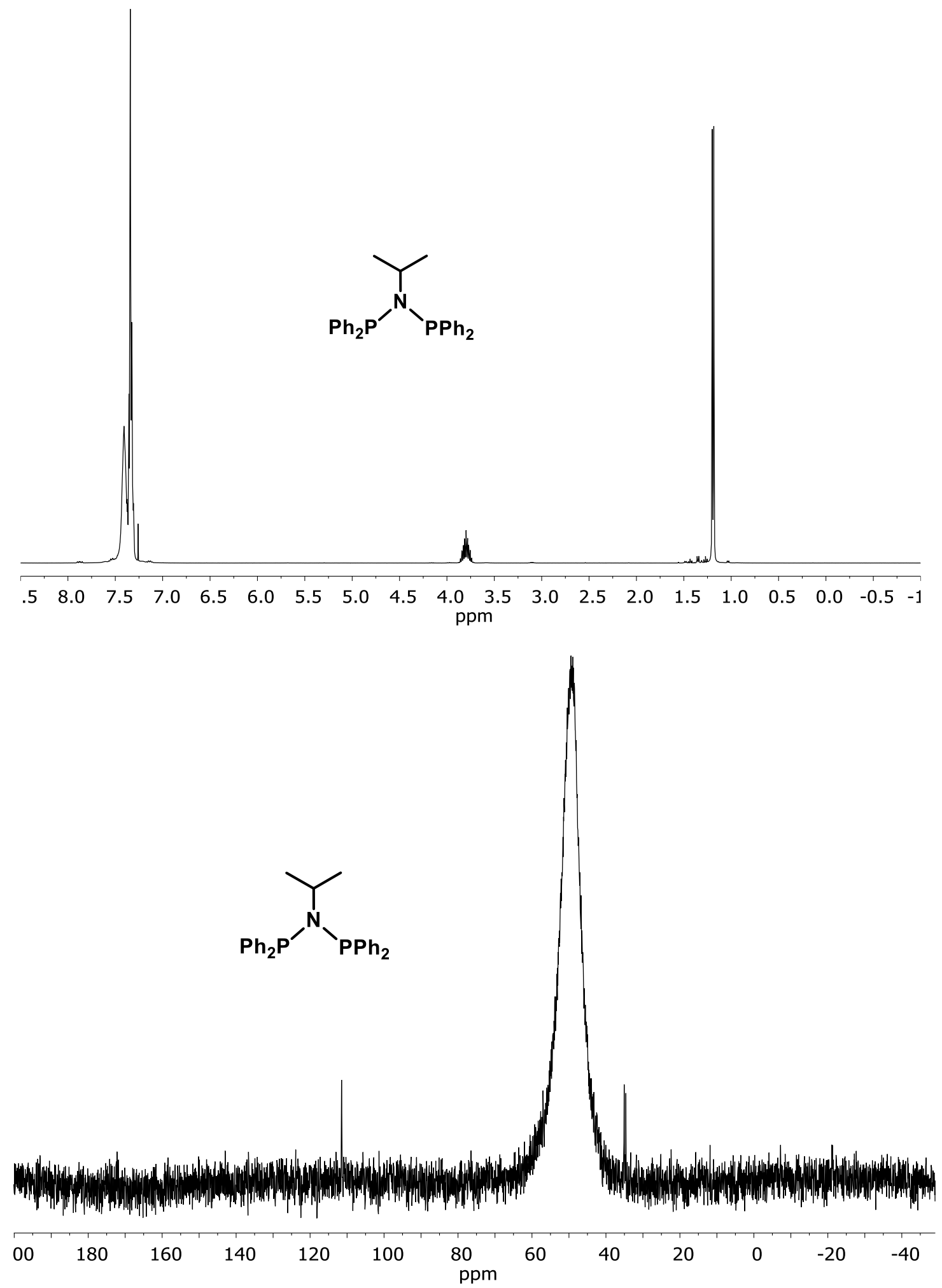
Figure S3. ${ }^{1} \mathrm{H}$ and ${ }^{31} \mathrm{P}$ NMR spectra of ligand 2 in $\mathrm{C}_{6} \mathrm{D}_{6}$; dppe, 1,2-bis(diphenylphosphino)ethane.
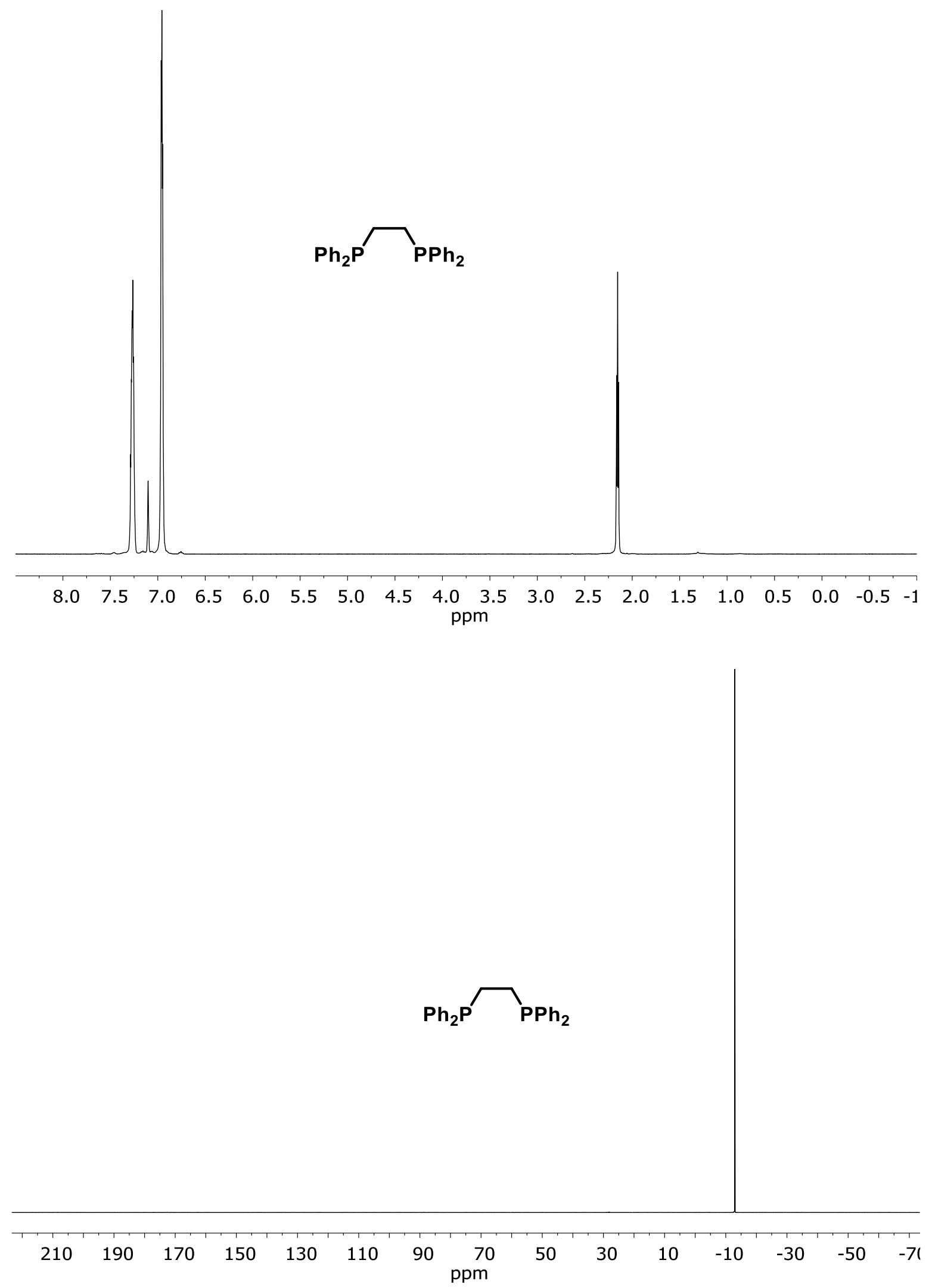
Figure S4. ${ }^{1} \mathrm{H}$ and ${ }^{31} \mathrm{P}$ NMR spectra of ligand 3 in $\mathrm{C}_{6} \mathrm{D}_{6} ; 1,1$ '-[(1R,2S)-1,2-dimethyl-1,2-ethanediyl] bis[1,1-diphenylphosphine].
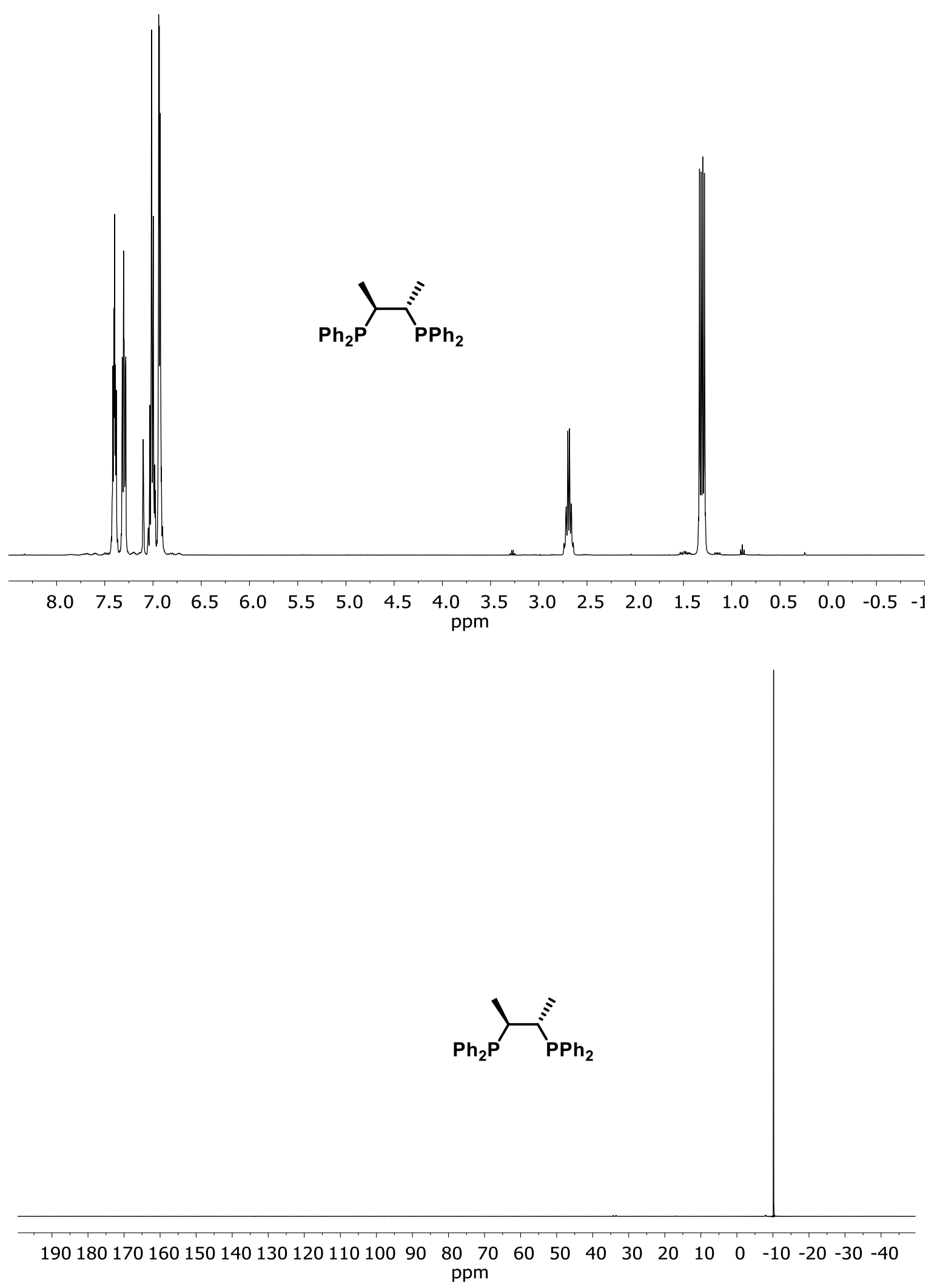
Figure S5. ${ }^{1} \mathrm{H}$ and ${ }^{31} \mathrm{P}$ NMR spectra of ligand 4 in $\mathrm{C}_{6} \mathrm{D}_{6} ; 1,1^{\prime}-(1 Z)$-1,2-ethenediylbis[1,1-diphenylphosphine].
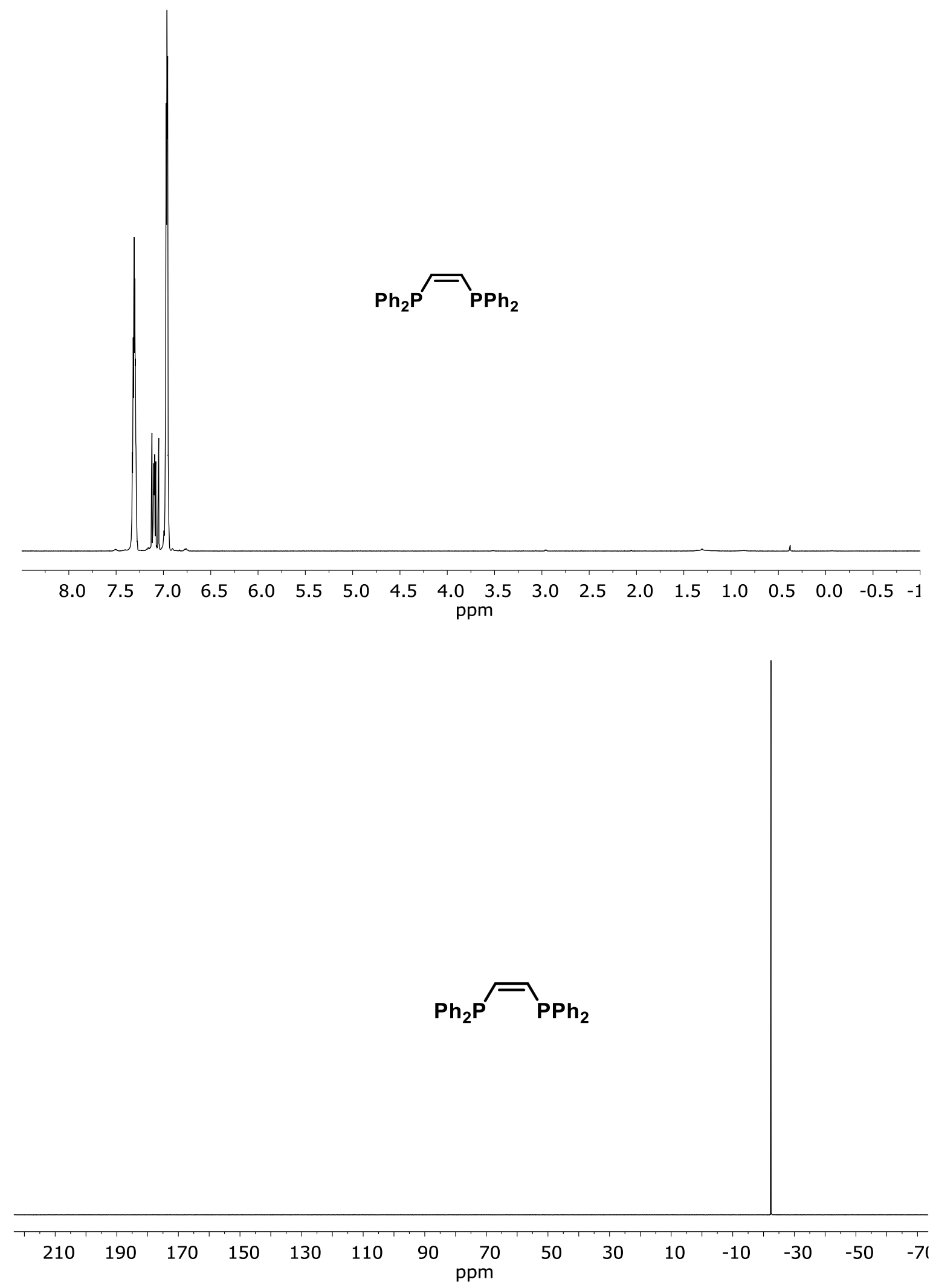
Figure S6. ${ }^{1} \mathrm{H}$ and ${ }^{31} \mathrm{P}$ NMR spectra of ligand 6 in $\mathrm{CDCl}_{3}$. 1,2-bis(diphenylphosphanyl)benzene.
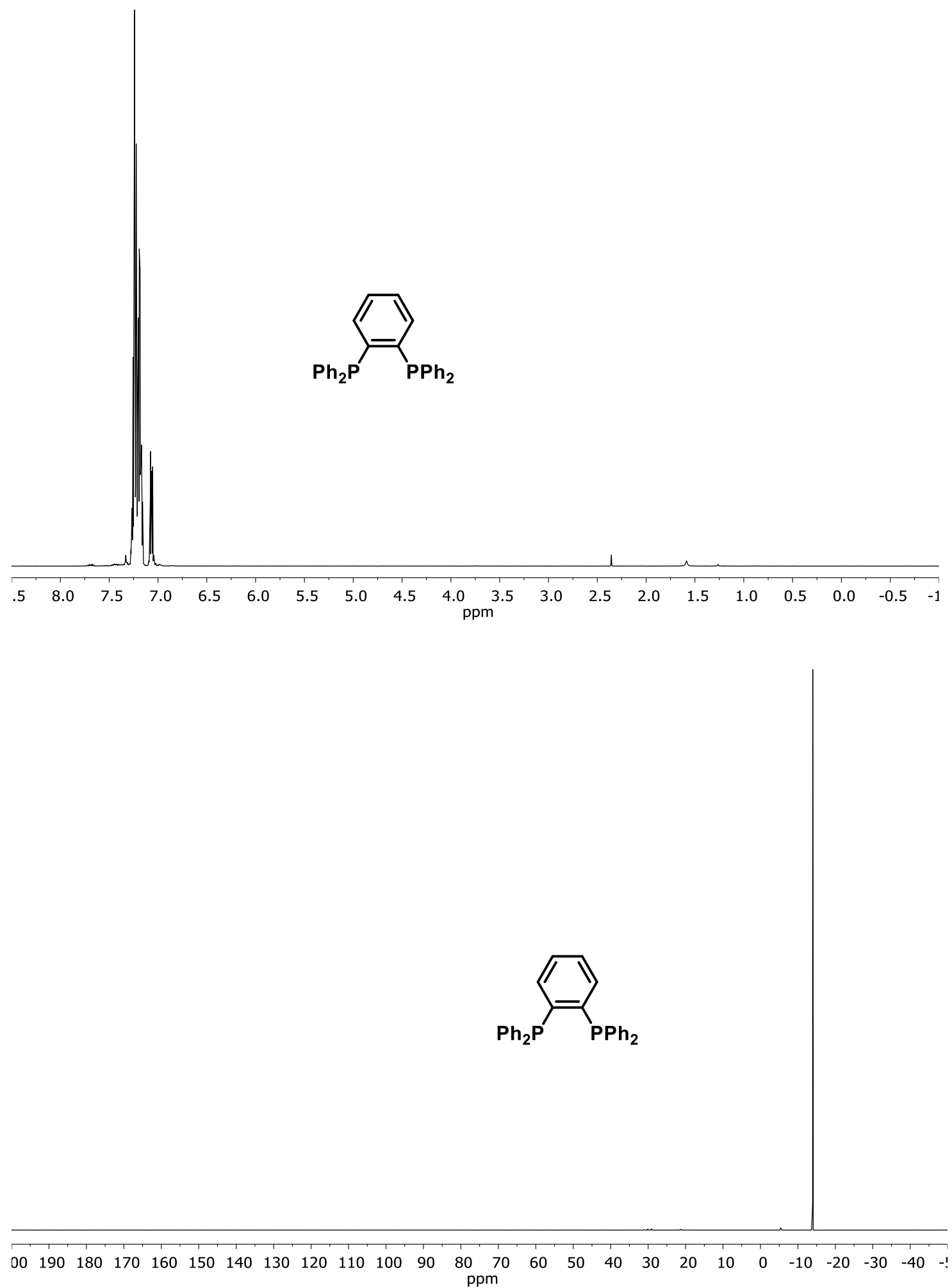
Figure S7. ${ }^{1} \mathrm{H}$ and ${ }^{31} \mathrm{P}$ NMR spectra of ligand $\mathbf{1 0}$ in $\mathrm{CDCl}_{3} ; N$-[bis(2-methoxyphenyl)phosphino]- $P, P$ bis(2-methoxyphenyl)-N-methylphosphinous amide.

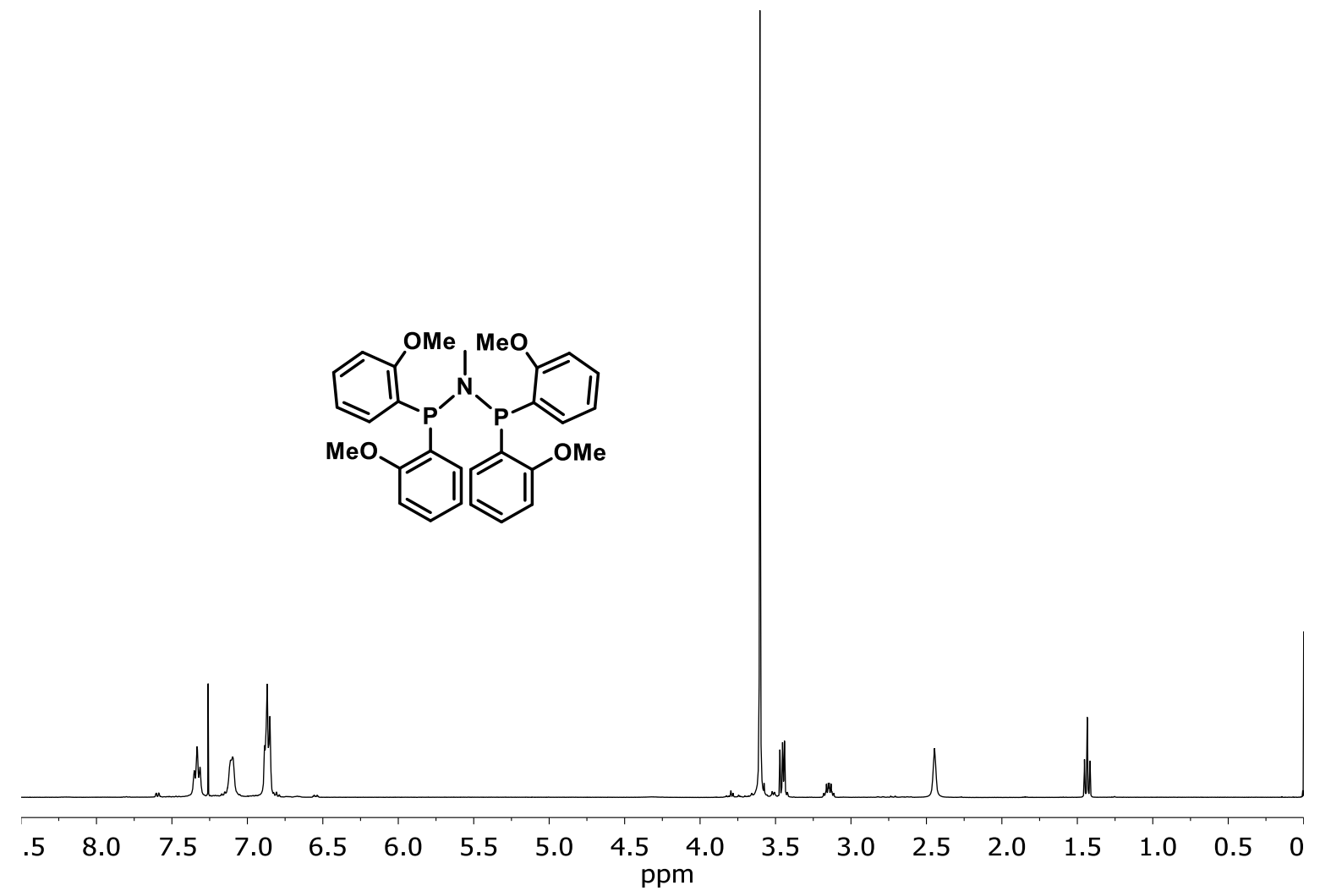<smiles>COc1ccccc1P(c1ccccc1OC)N(C)P(c1ccccc1OC)c1ccccc1OC</smiles>

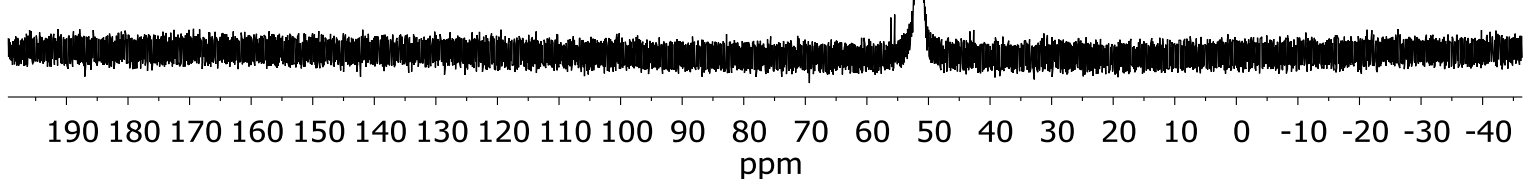


Figure S8. ${ }^{1} \mathrm{H}$ and ${ }^{31} \mathrm{P}$ NMR spectra of ligand 11 in $\mathrm{C}_{6} \mathrm{D}_{6} .1$ 1,1'-(1,2-ethanediyl)bis[1-(2-methoxyphenyl)-1phenyl-phosphine.
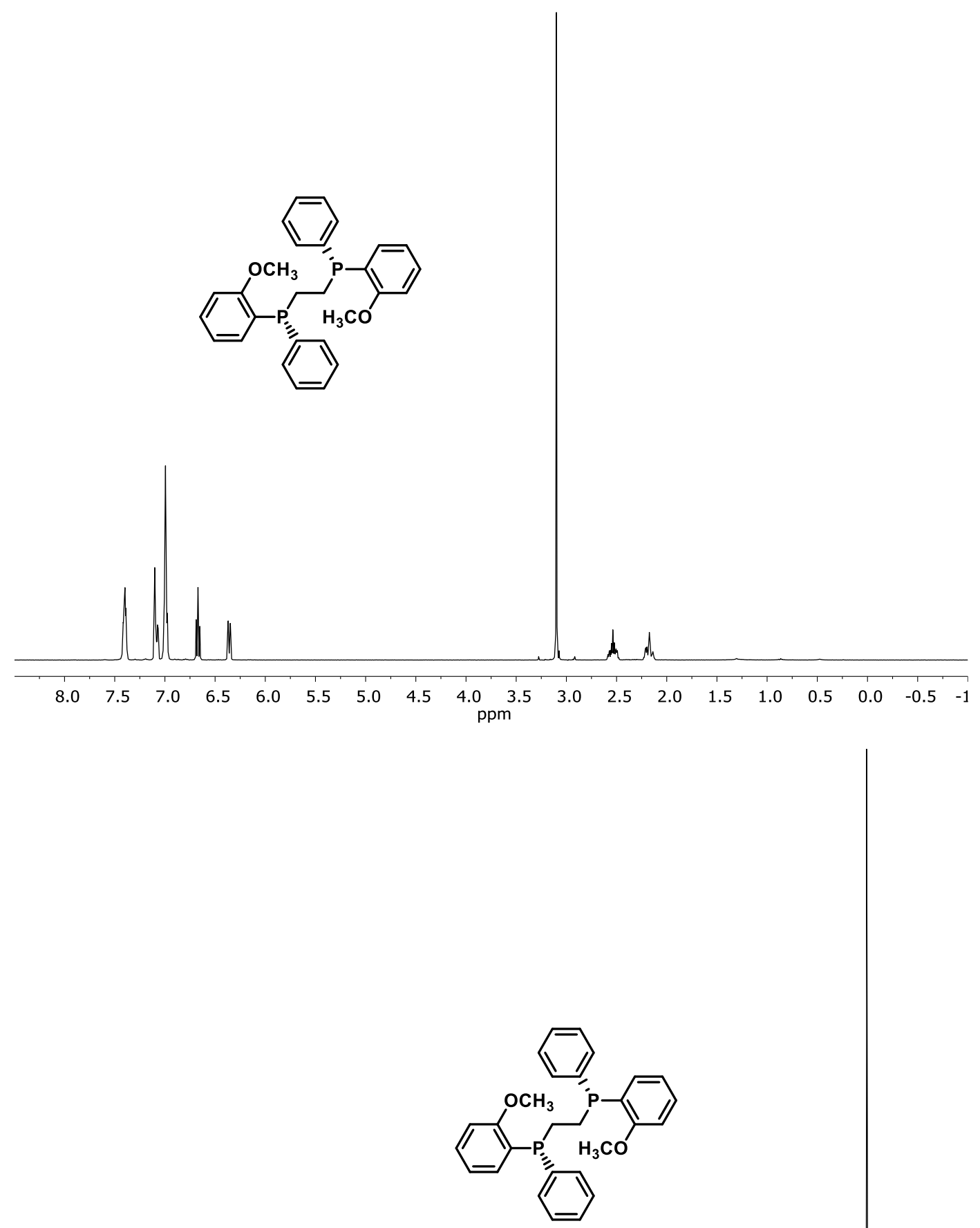

$\begin{array}{lllllllllllllllllllllll}180 & 170 & 160 & 150 & 140 & 130 & 120 & 110 & 100 & 90 & 80 & \begin{array}{c}70 \\ \mathrm{ppm}\end{array} & 60 & 50 & 40 & 30 & 20 & 10 & 0 & -10 & -20 & -30 & -40\end{array}$ 
Figure S9. ${ }^{1} \mathrm{H}$ and ${ }^{31} \mathrm{P}$ NMR spectra of ligand 12 in $\mathrm{C}_{6} \mathrm{D}_{6}$; $\left(1 R, 1^{\prime} R\right)-1,1^{\prime}-(1,2$-ethanediyl)bis[1-(2methylphenyl)-1-phenylphosphine].
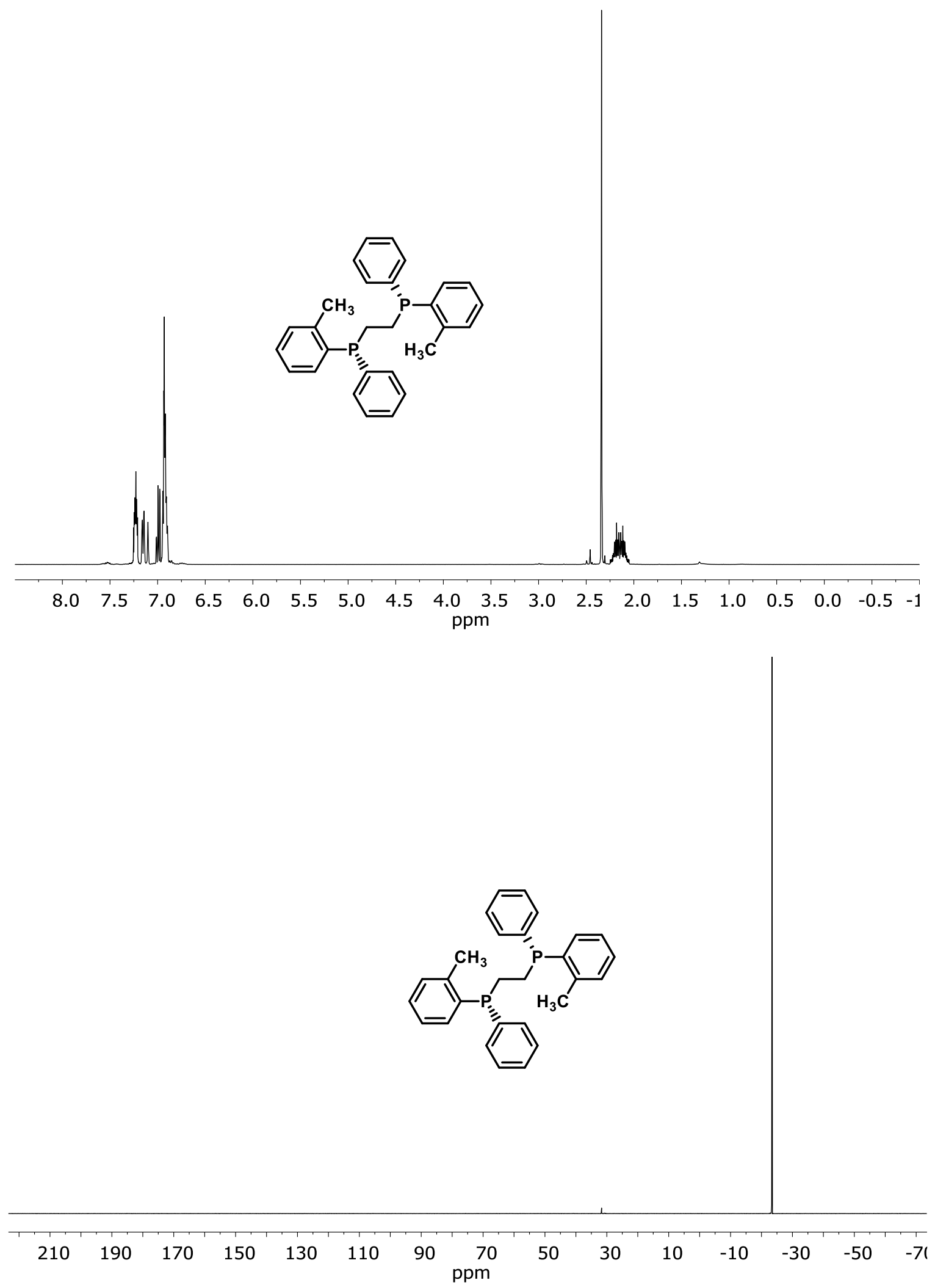
Figure S10. ${ }^{1} \mathrm{H}$ and ${ }^{31} \mathrm{P}$ NMR spectra of ligand 13 in $\mathrm{CDCl}_{3} ; 2,2$,2",2"'-(1,2-ethanediyldiphosphinidyne) tetrakis-pyridine.
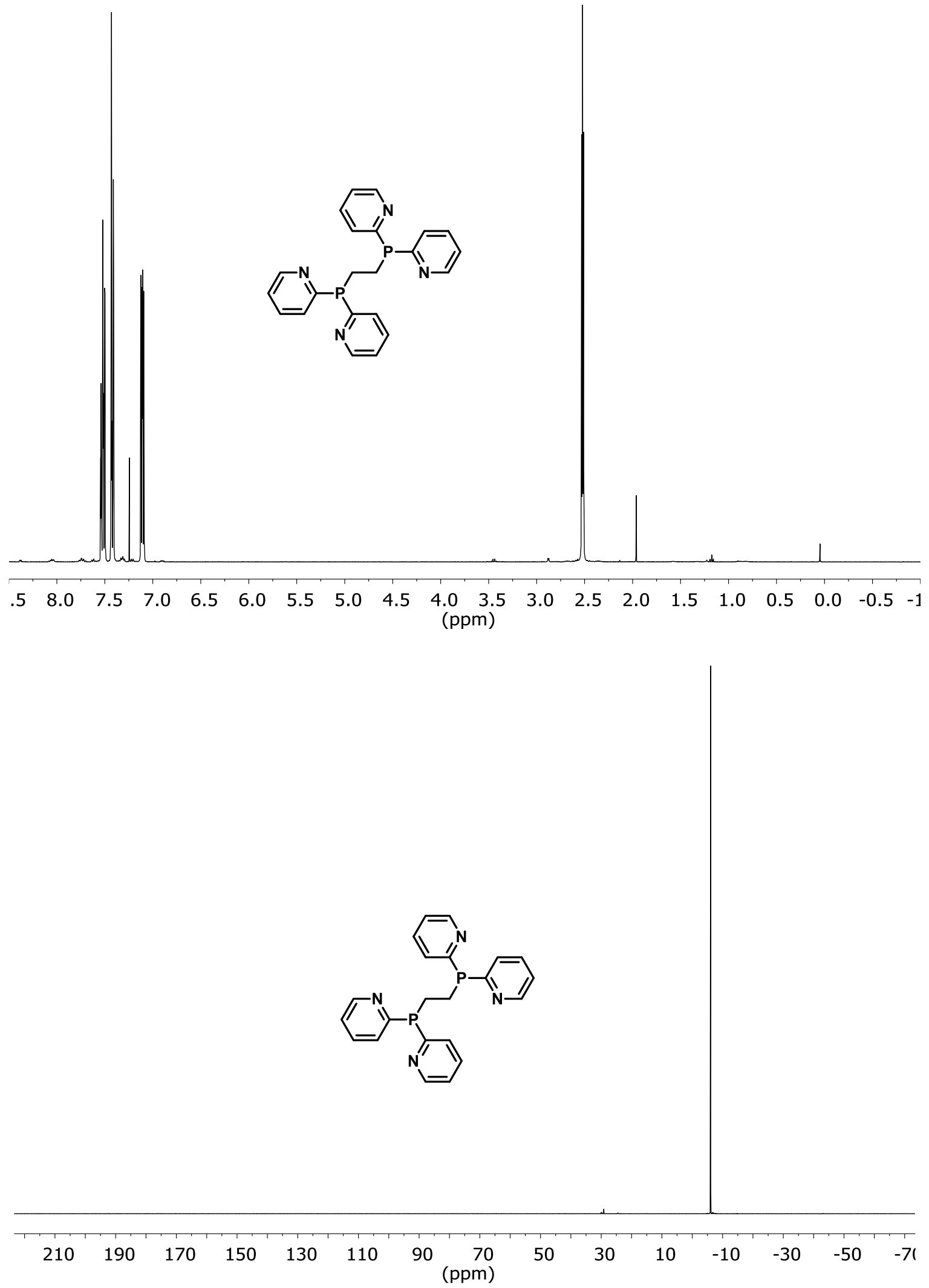
Figure $\mathrm{S} 11 .{ }^{1} \mathrm{H}$ and ${ }^{31} \mathrm{P}$ NMR spectra of ligand 14 in $\mathrm{CDCl}_{3} ;(1 R)$-[1,1'-binaphthalene]-2,2'-diylbis [diphenylphosphine].
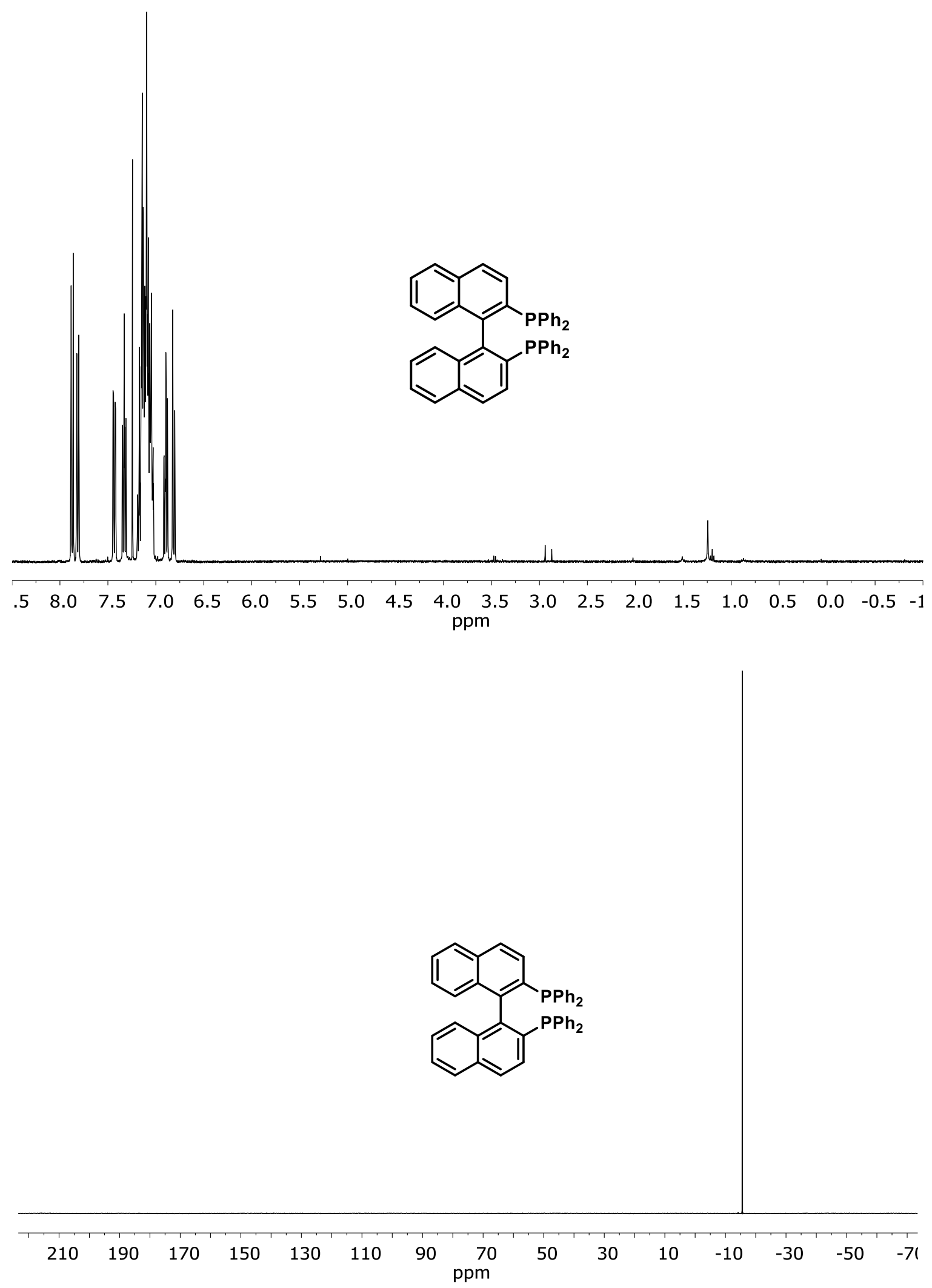
Figure S12. ${ }^{1} \mathrm{H}$ and ${ }^{31} \mathrm{P}$ NMR spectra of ligand 15 in $\mathrm{CDCl}_{3} ; 1,1^{\prime}-[(1 \mathrm{R})-6,6$ '-dimethoxy[1,1'-biphenyl]2,2'-diyl]bis[1,1-di-2-furanylphosphine].
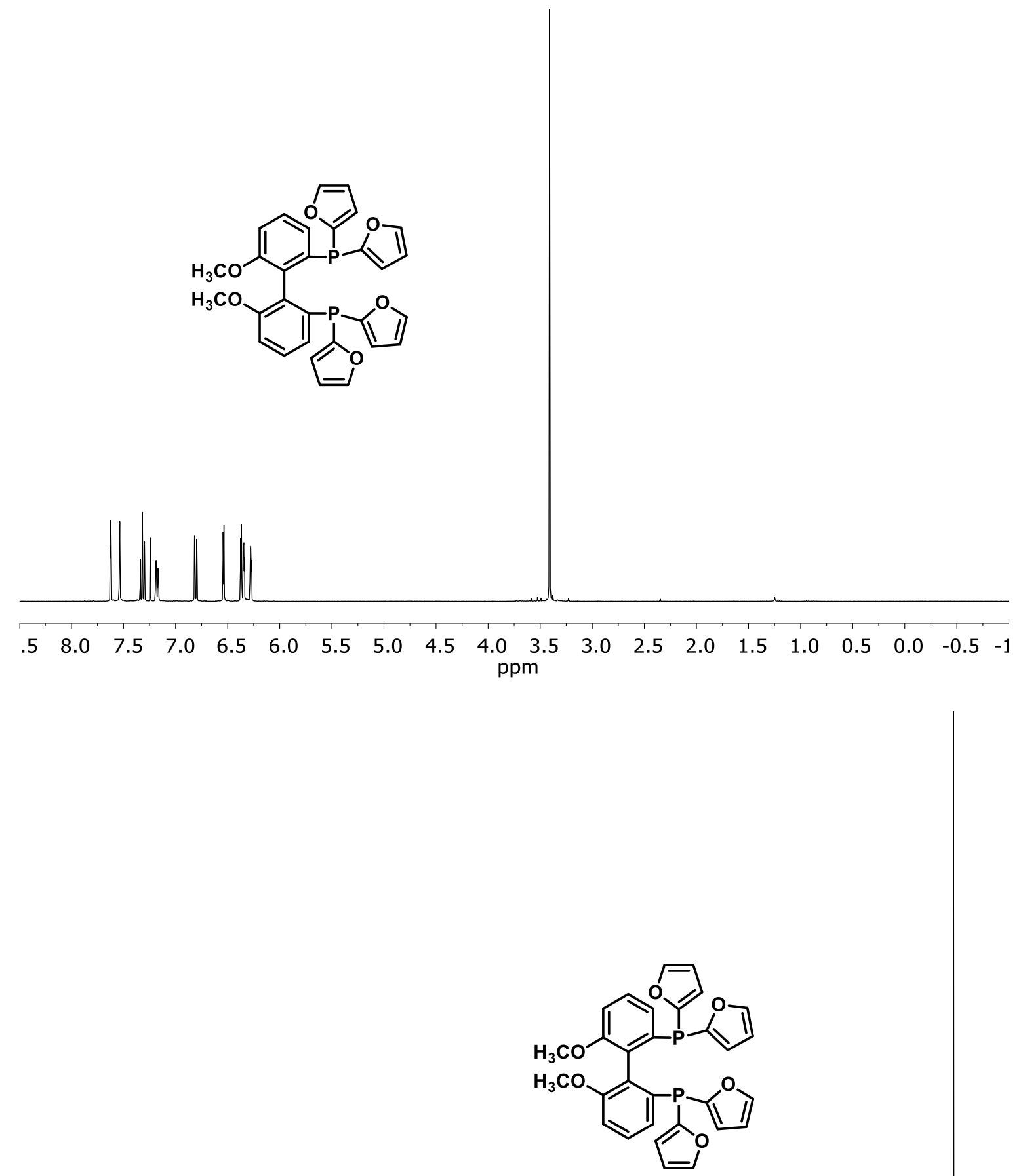
Figure S13. ${ }^{1} \mathrm{H}$ and ${ }^{31} \mathrm{P}$ NMR spectra of ligand 16 in $\mathrm{CDCl}_{3} ; 1,1^{\prime}-(9,9$ ', 10,10'-tetrahydro[11,11'-bi-9,10ethenoanthracene]-12,12'-diyl)bis[1,1-diphenylphosphine].
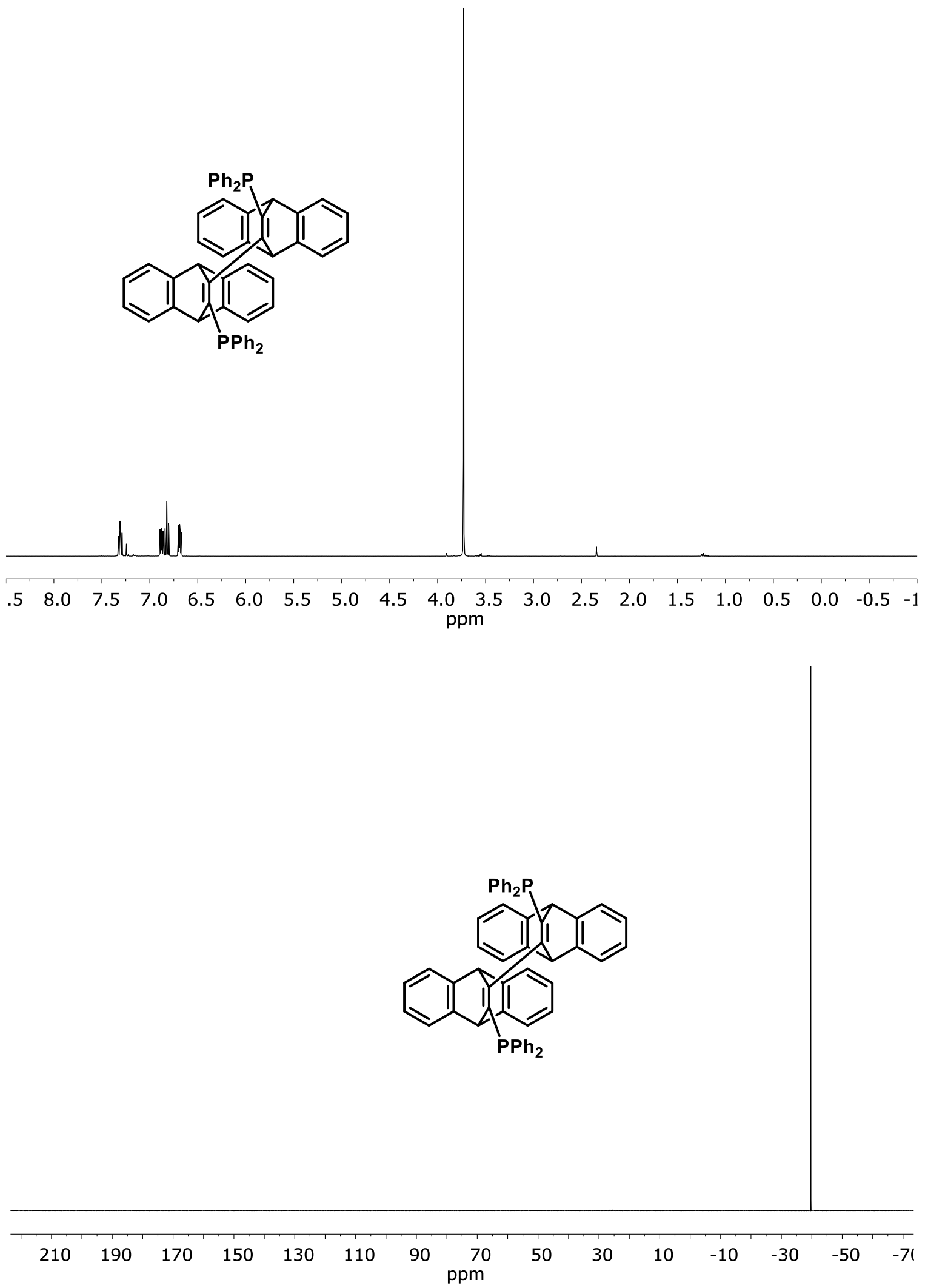
Figure S14. ${ }^{1} \mathrm{H}$ and ${ }^{31} \mathrm{P}$ NMR spectra of ligand 17 in $\mathrm{C}_{6} \mathrm{D}_{6}$; $(R)-(-)-1-[(S)-2-$ (diphenylphosphino)ferrocenyl]ethyldi-3,5-xylylphosphine.
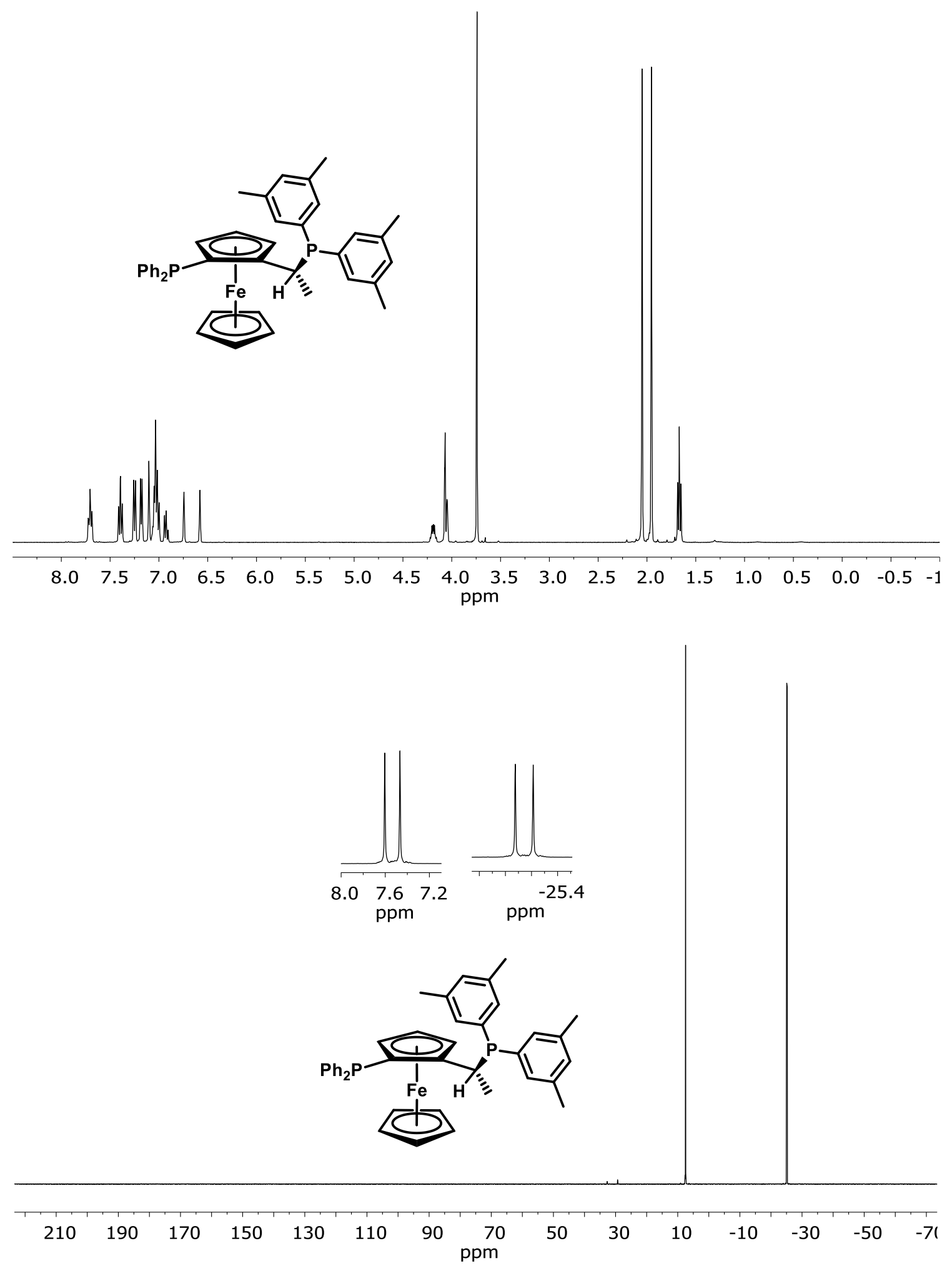
Figure S15. ${ }^{1} \mathrm{H}$ and ${ }^{31} \mathrm{P}$ NMR spectra of ligand 18 in $\mathrm{C}_{6} \mathrm{D}_{6}$; Xantphos, 1,1'-(9,9-dimethyl-9H-xanthene-4, 5-diyl)bis[1,1-diphenylphosphine].

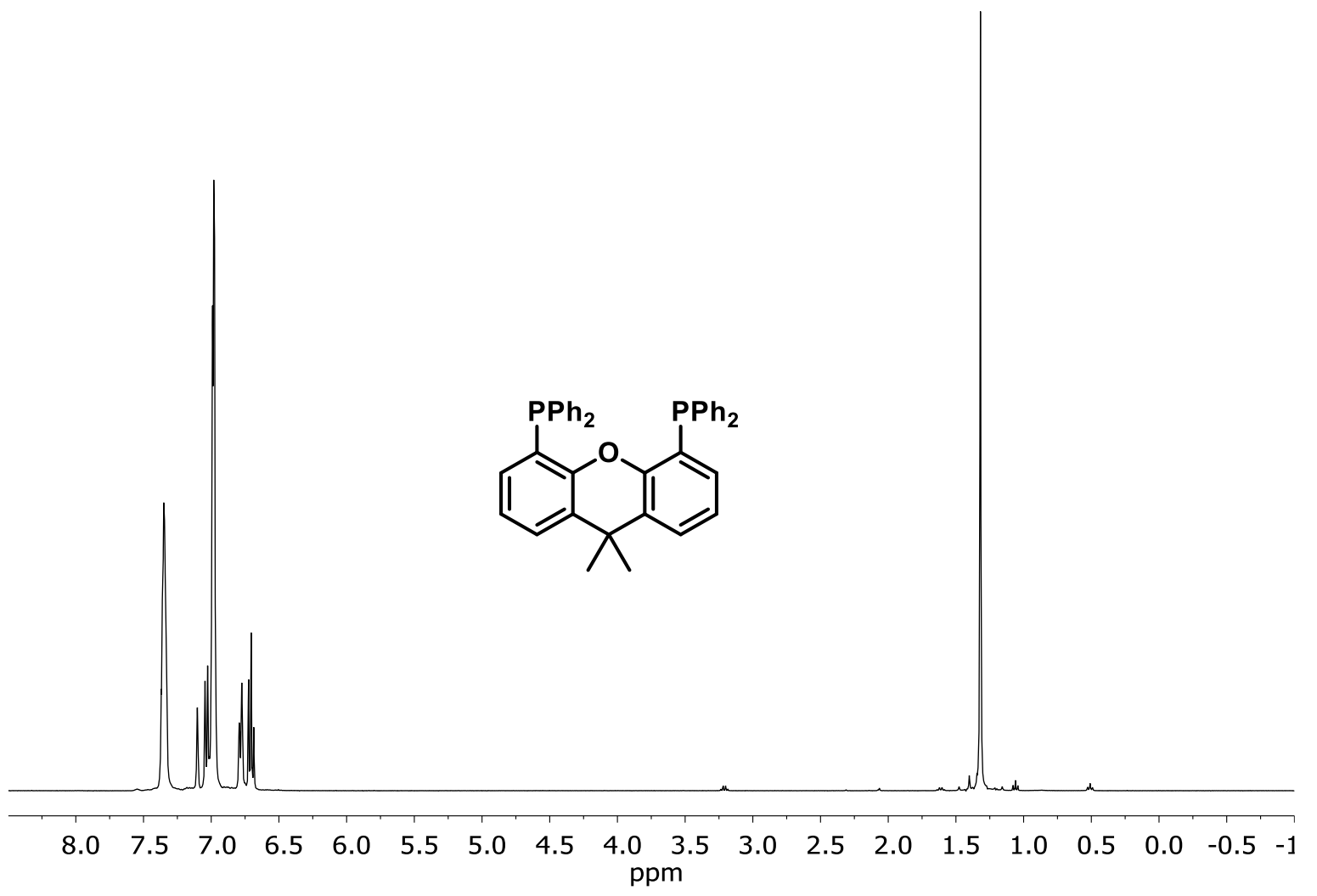<smiles>CCCCCc1cccc2c1Oc1c(P)cccc1C2(C)C</smiles> 
Figure S16. ${ }^{1} \mathrm{H}$ and ${ }^{31} \mathrm{P}$ NMR spectra of ligand 19 in $\mathrm{C}_{6} \mathrm{D}_{6} ; 1,1$-(1,2-ethanediyl)bis[1,1-dimethyl phosphine].
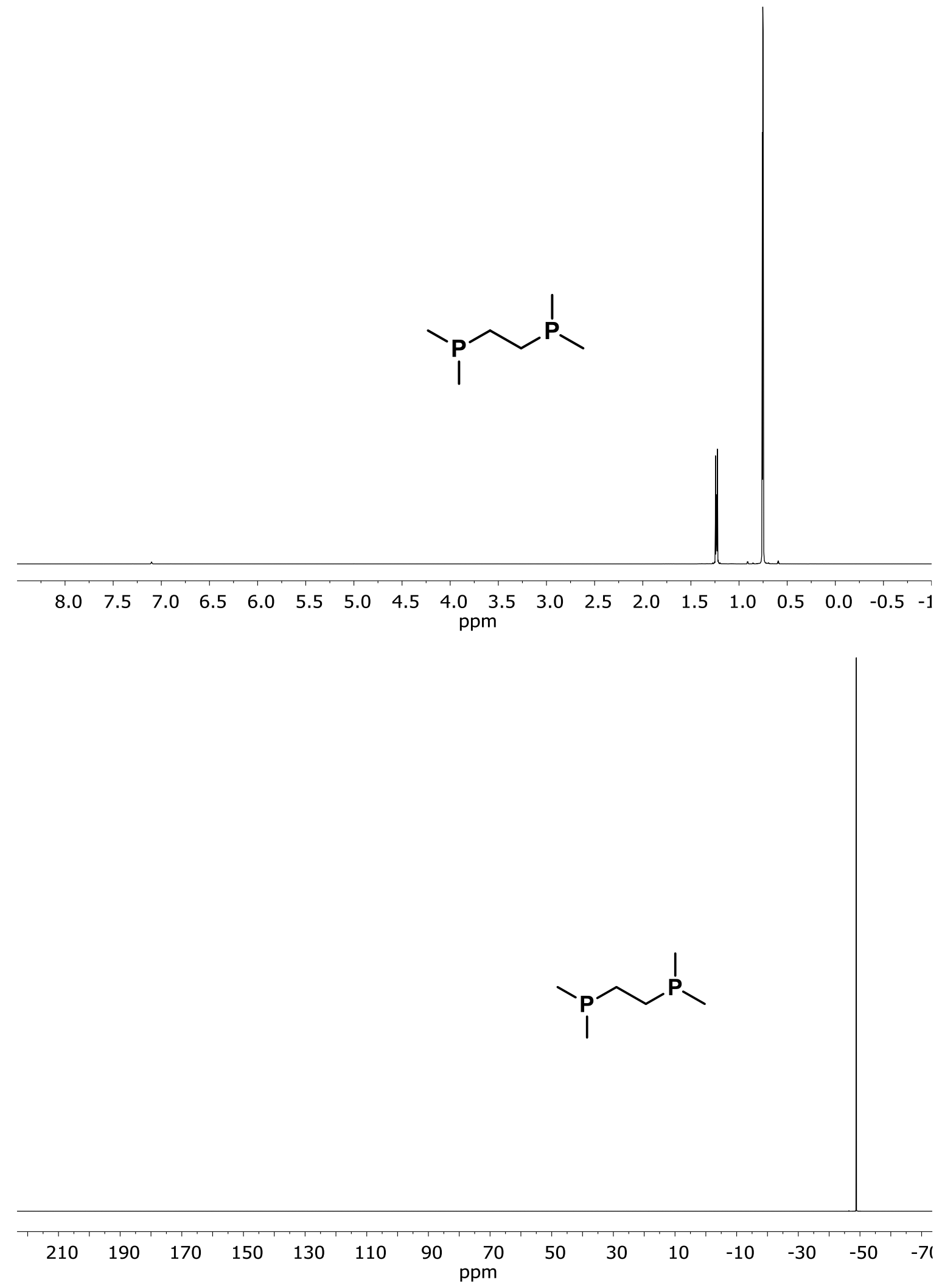
Figure S17. ${ }^{1} \mathrm{H}$ and ${ }^{31} \mathrm{P}$ NMR spectra of ligand 20 in $\mathrm{C}_{6} \mathrm{D}_{6} ; 1,1$ '-(1,2-ethanediyl)bis[1,1-diethylphosphine].
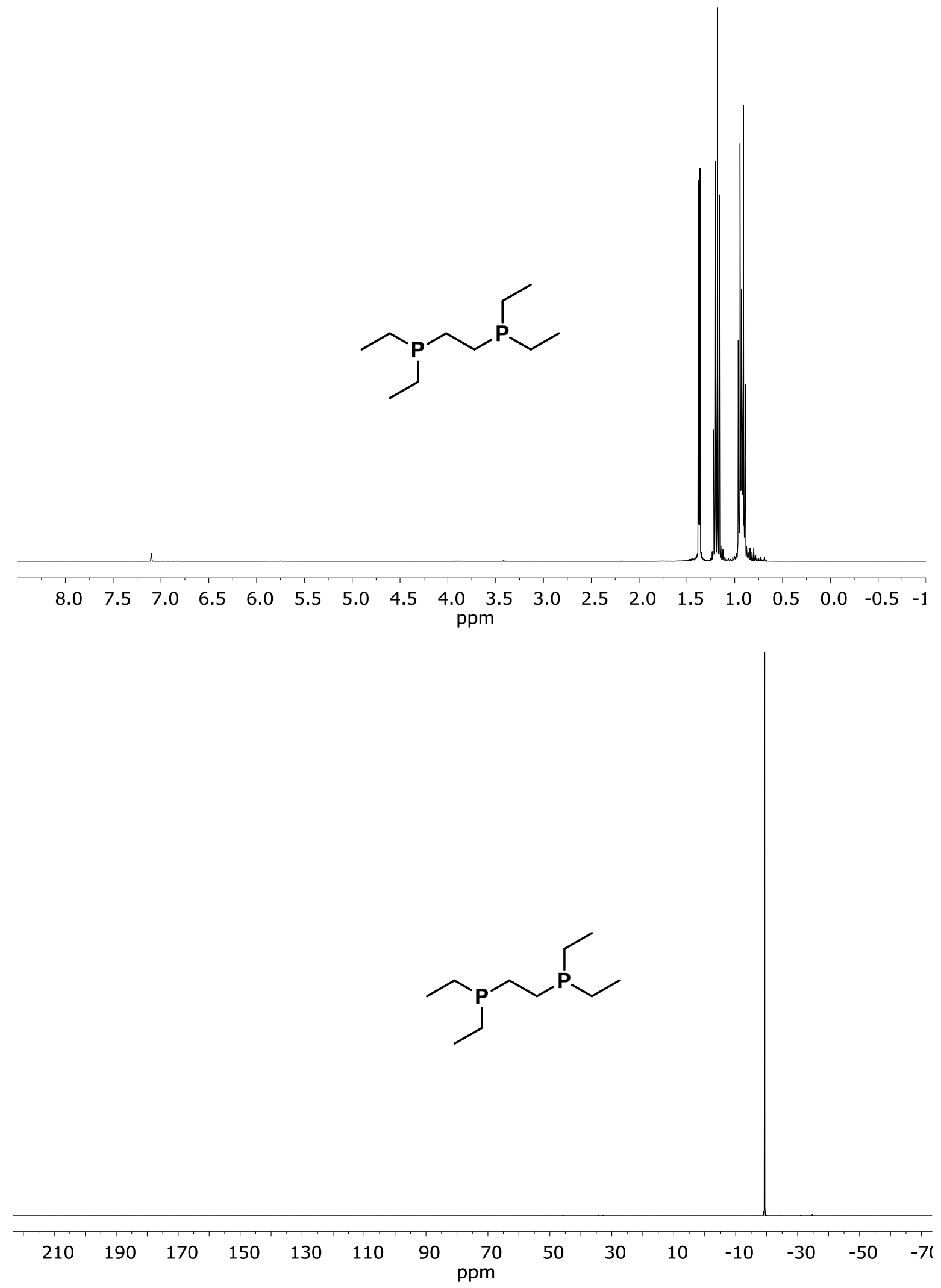
Figure S18. ${ }^{1} \mathrm{H}$ and ${ }^{31} \mathrm{P}$ NMR spectra of ligand 21 in $\mathrm{C}_{6} \mathrm{D}_{6} ; 1,1$-(1,2-ethanediyl)bis[1,1-bis(1-methylethyl) phosphine].
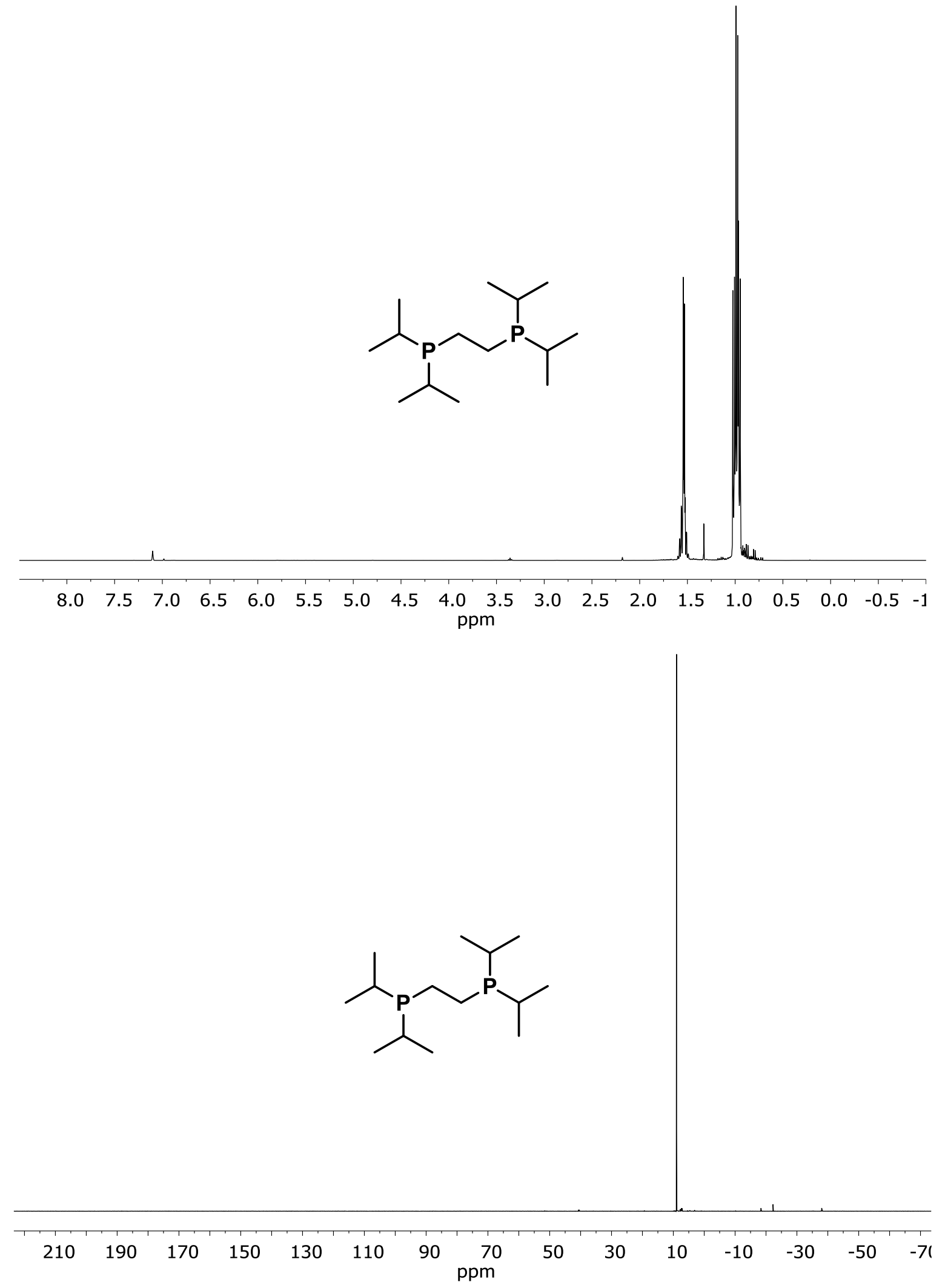
Figure S19. ${ }^{1} \mathrm{H}$ and ${ }^{31} \mathrm{P}$ NMR spectra of ligand 22 in $\mathrm{CDCl}_{3} ; 1,1$ '-(1,2-phenylene)bis[1,1-dimethylphosphine].
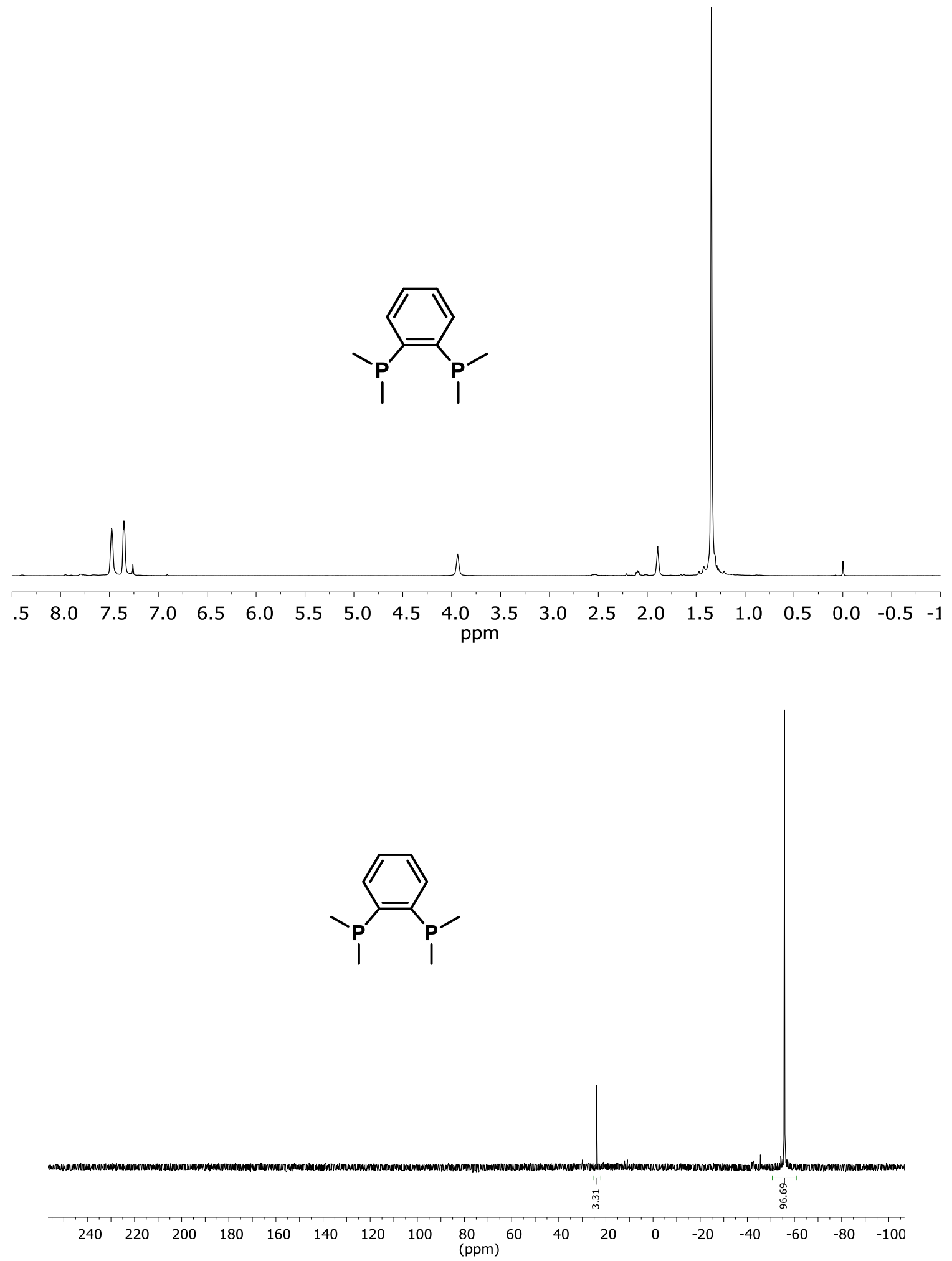
Figure S20. ${ }^{1} \mathrm{H}$ and ${ }^{31} \mathrm{P}$ NMR spectra of ligand 23 in $\mathrm{CDCl}_{3} ; 1,1$ '-(1,2-phenylene)bis[1,1-diethylphosphine].
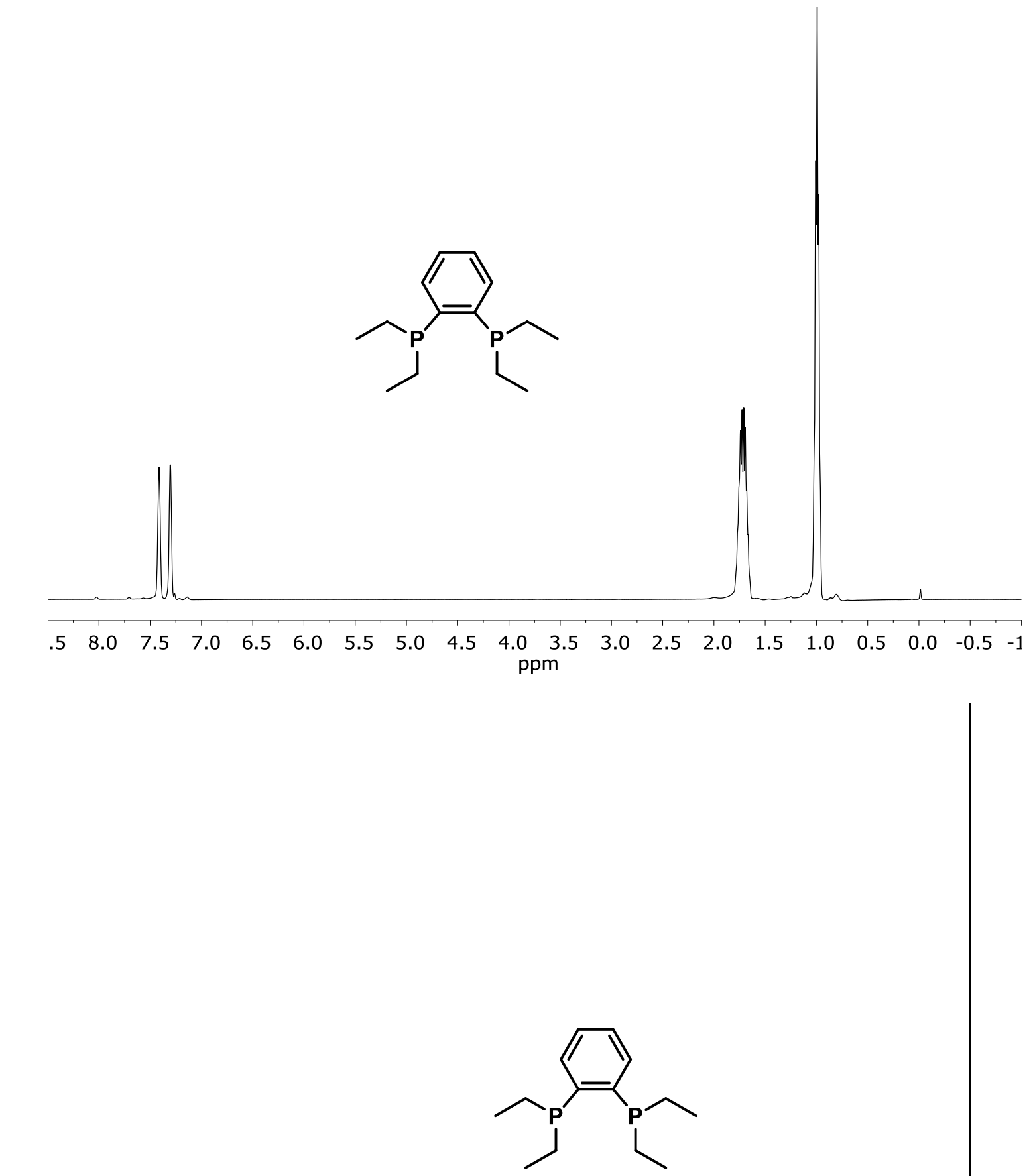
Figure S21. ${ }^{1} \mathrm{H}$ and ${ }^{31} \mathrm{P}$ NMR spectra of ligand 24 in $\mathrm{CDCl}_{3}$; 1,1'-(1,2-phenylene)bis[1,1-bis(1methylethyl)phosphine]

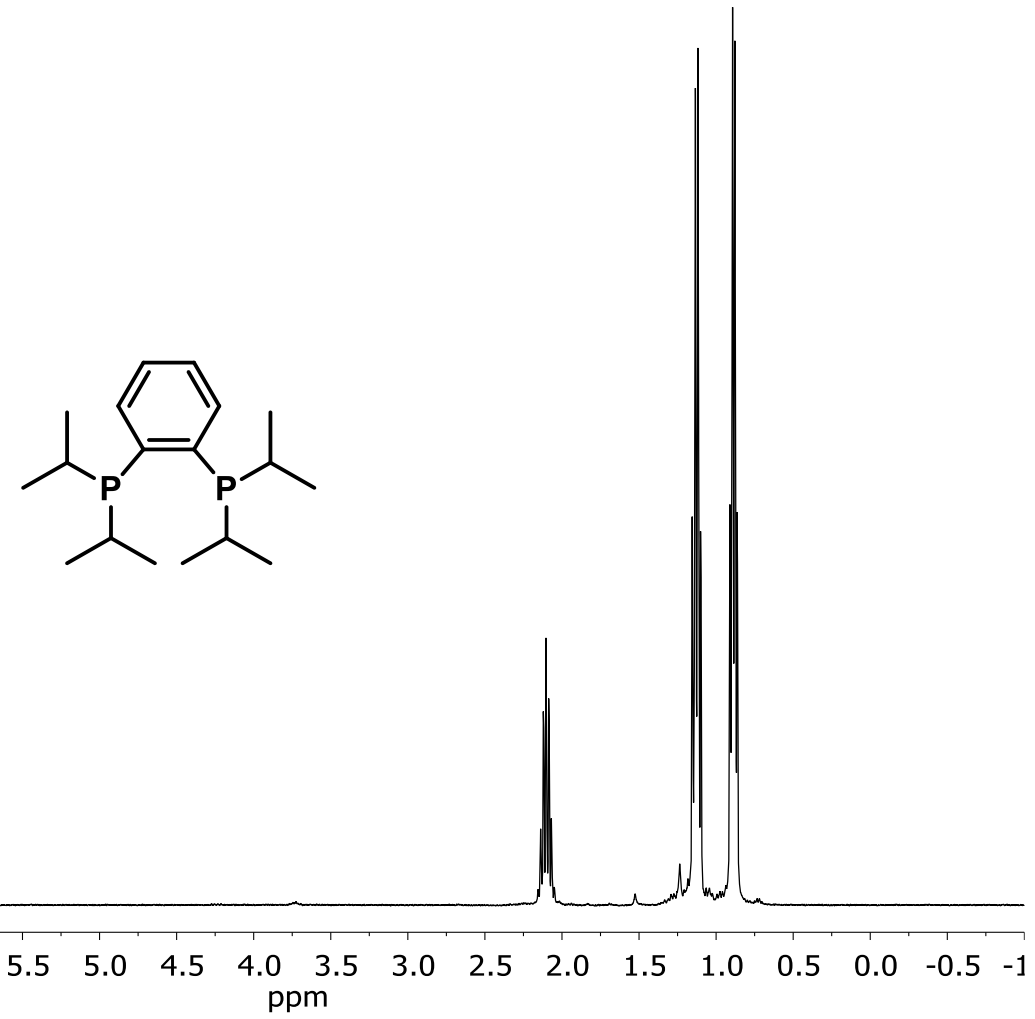

$\begin{array}{llllllllllllllllllll}.5 & 8.0 & 7.5 & 7.0 & 6.5 & 6.0 & 5.5 & 5.0 & 4.5 & \underset{\mathrm{ppm}}{4.0} & 3.5 & 3.0 & 2.5 & 2.0 & 1.5 & 1.0 & 0.5 & 0.0 & -0.5 & -1\end{array}$

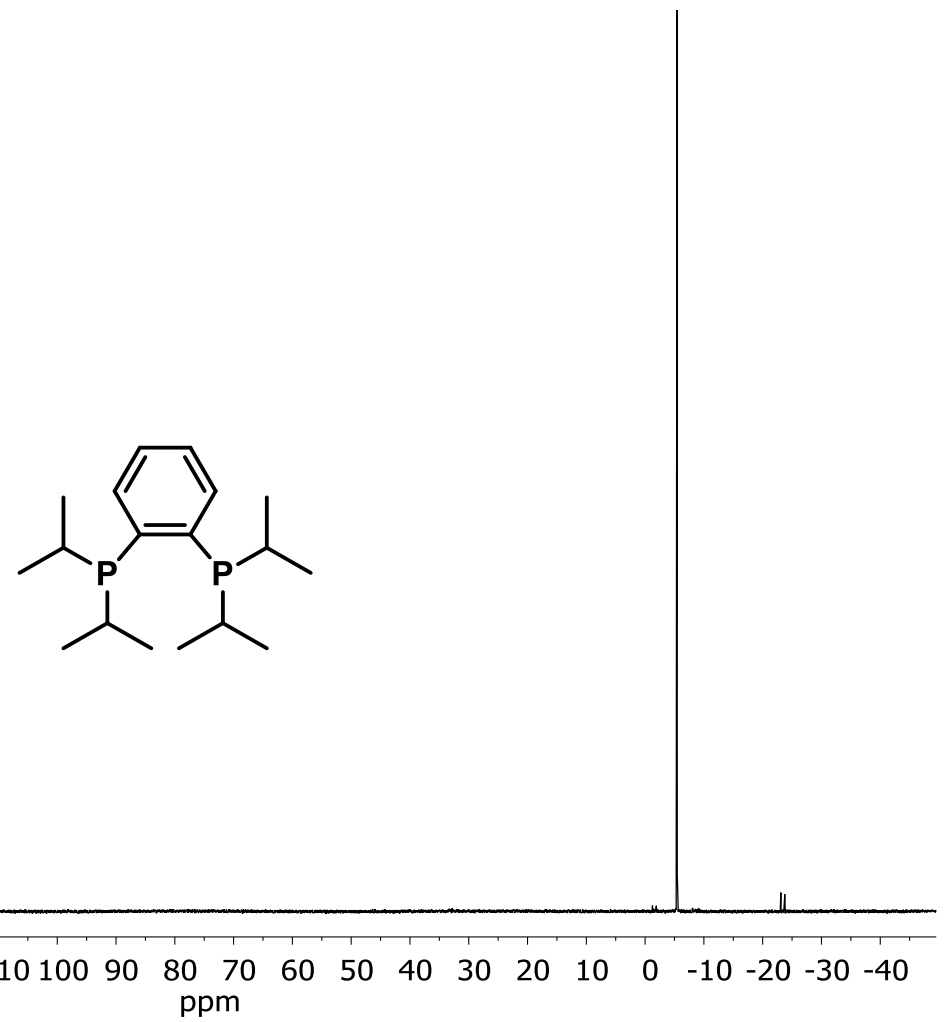


Figure S22. ${ }^{1} \mathrm{H}$ and ${ }^{31} \mathrm{P}$ NMR spectra of ligand 25 in $\mathrm{C}_{6} \mathrm{D}_{6}$. 1,2-bis(tert-butyl(methyl)phosphanyl)benzene.
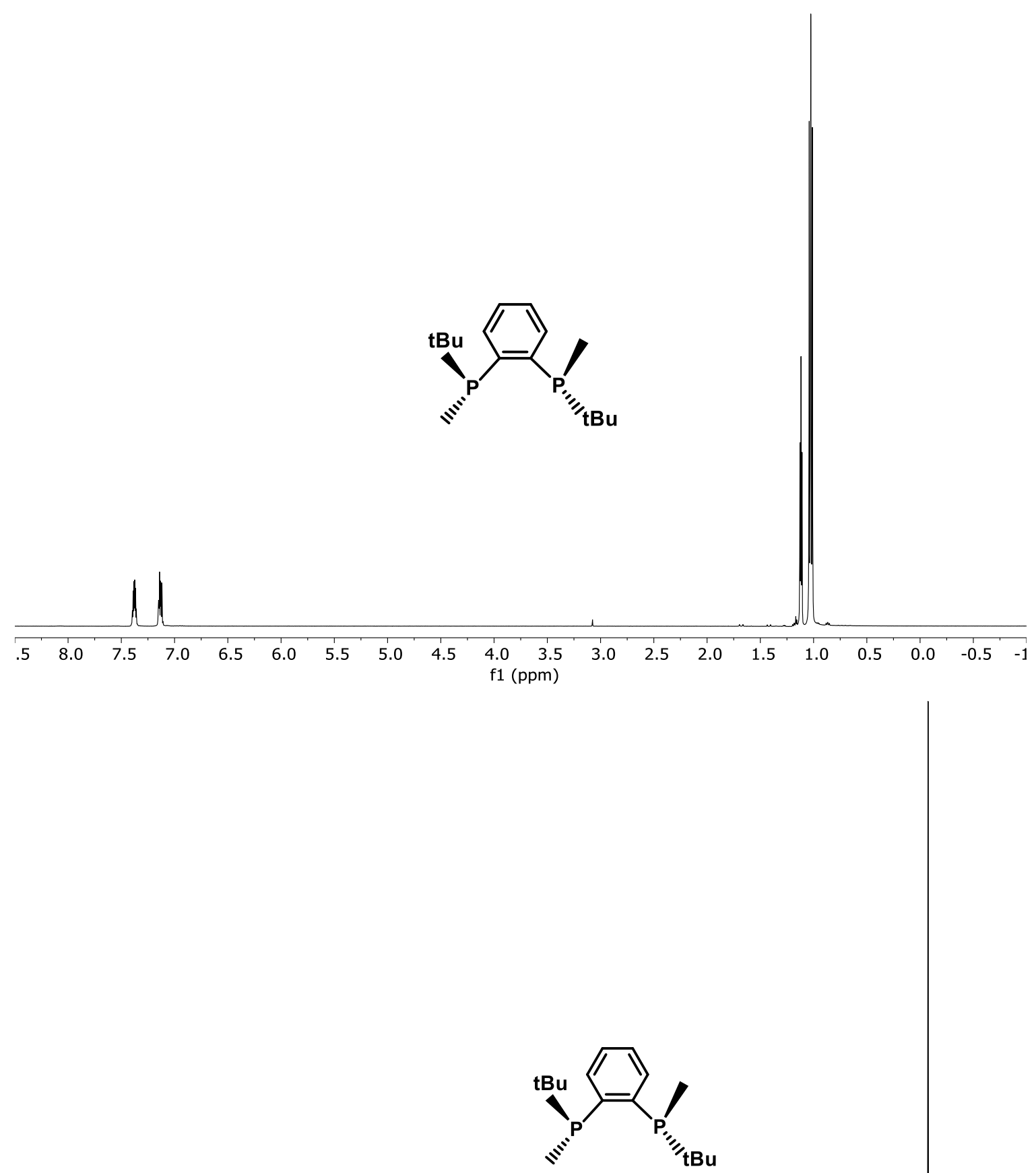

\begin{tabular}{lllllllllllllllllllllllllllll}
\hline 00 & 190 & 180 & 170 & 160 & 150 & 140 & 130 & 120 & 110 & 100 & 90 & 80 & 70 & 60 & 50 & 40 & 30 & 20 & 10 & 0 & -10 & -20 & -30 & -40 & $-!$
\end{tabular} 
Figure S23. ${ }^{1} \mathrm{H}$ and ${ }^{31} \mathrm{P}$ NMR spectra of ligand 26 in $\mathrm{C}_{6} \mathrm{D}_{6} ; 2,3$-bis $[(S)-(1,1$-dimethylethyl $)$ methylphosphino]-quinoxaline.

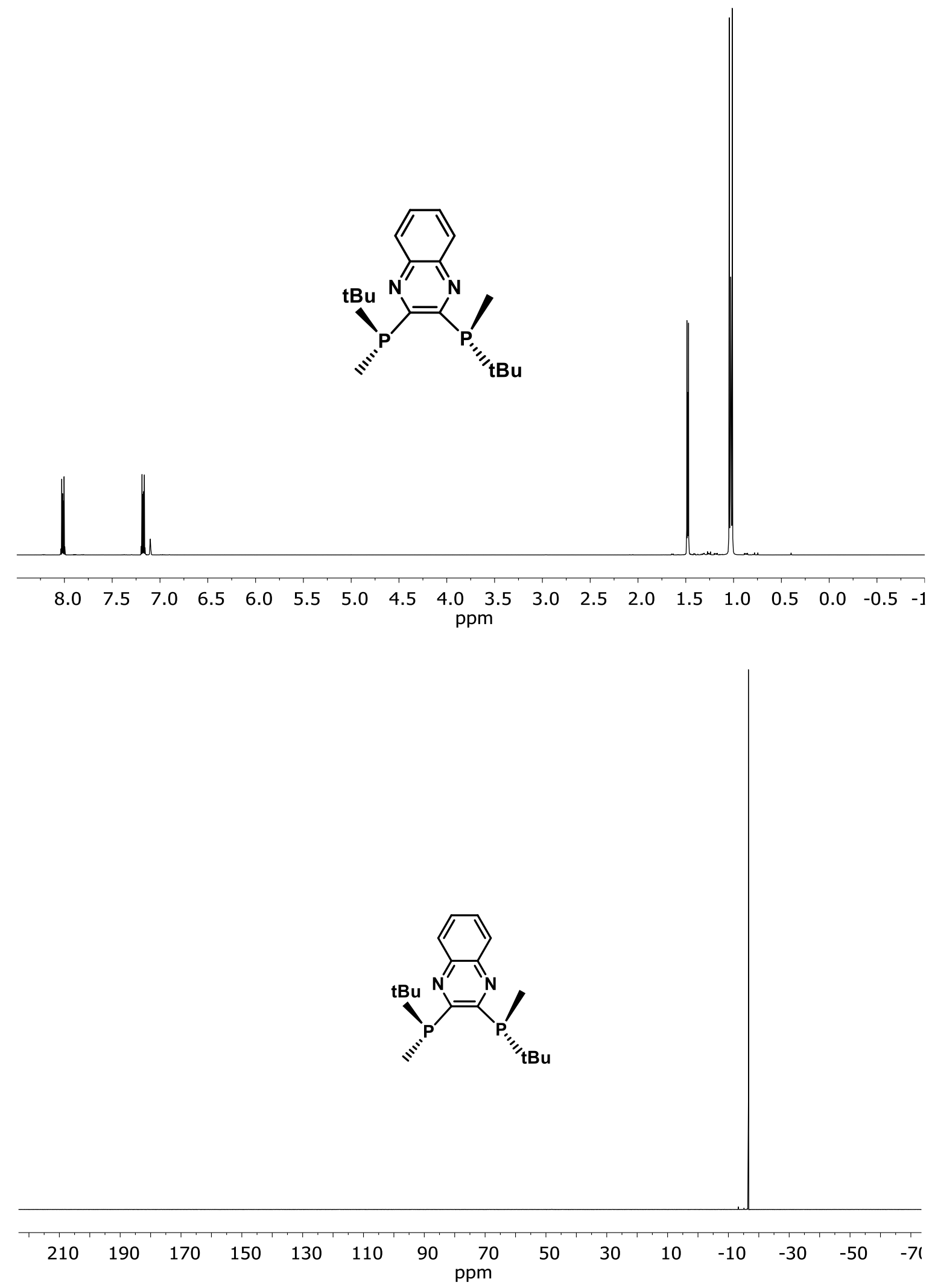


Figure S24. ${ }^{1} \mathrm{H}$ and ${ }^{31} \mathrm{P}$ NMR spectra of ligand 27 in $\mathrm{C}_{6} \mathrm{D}_{6}$; MeDuPhos, $\left(2 R, 2^{\prime} R, 5 R, 5^{\prime} R\right)-1,1^{\prime}-(1,2-$ phenylene)bis[2,5-dimethylphospholane].
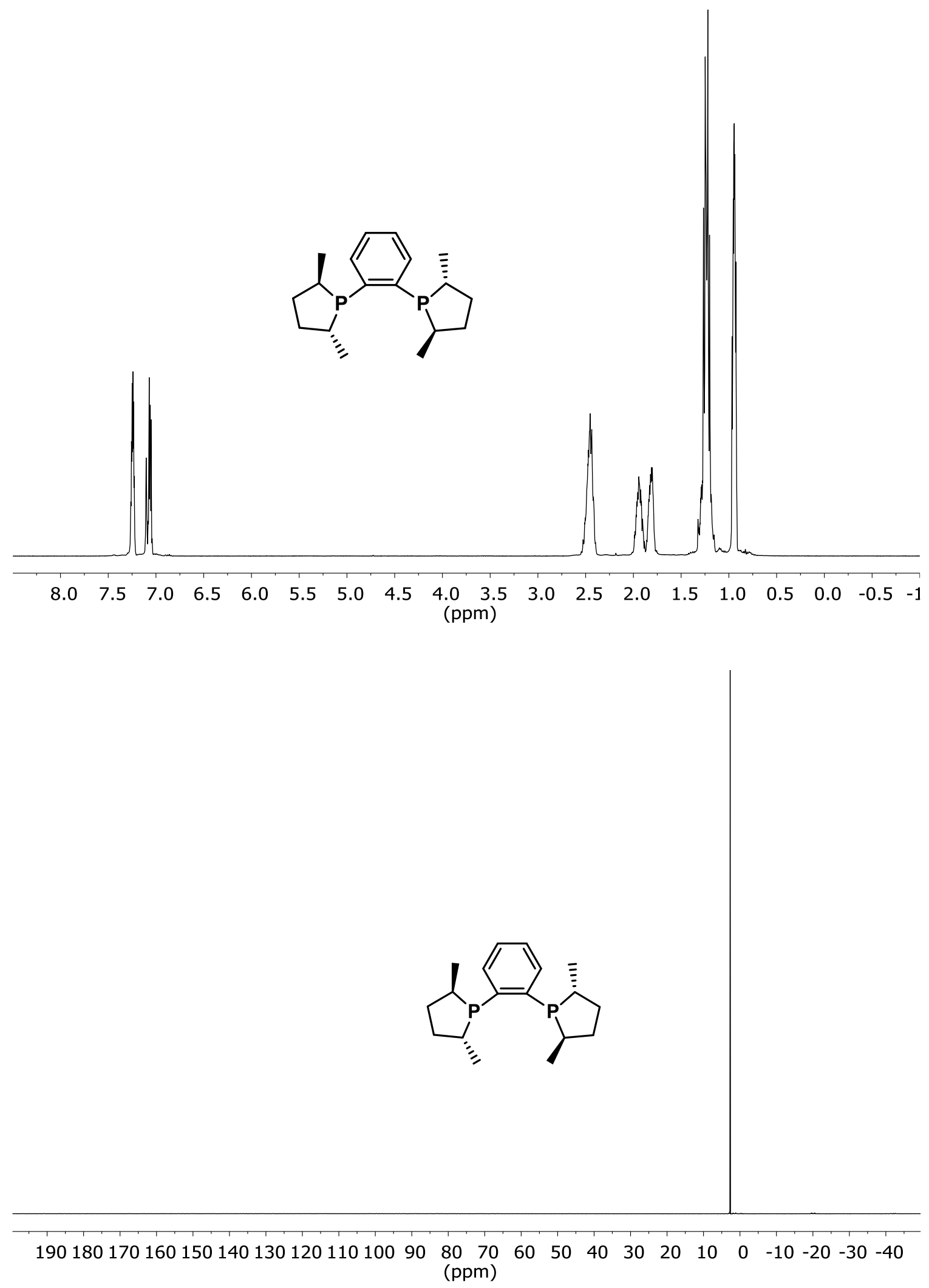
Figure S25. ${ }^{1} \mathrm{H}$ and ${ }^{31} \mathrm{P}$ NMR spectrum of ligand 28 in $\mathrm{CDCl}_{3} ; N$-(diphenylphosphino)- $P, P$-diphenylphosphinous amide.
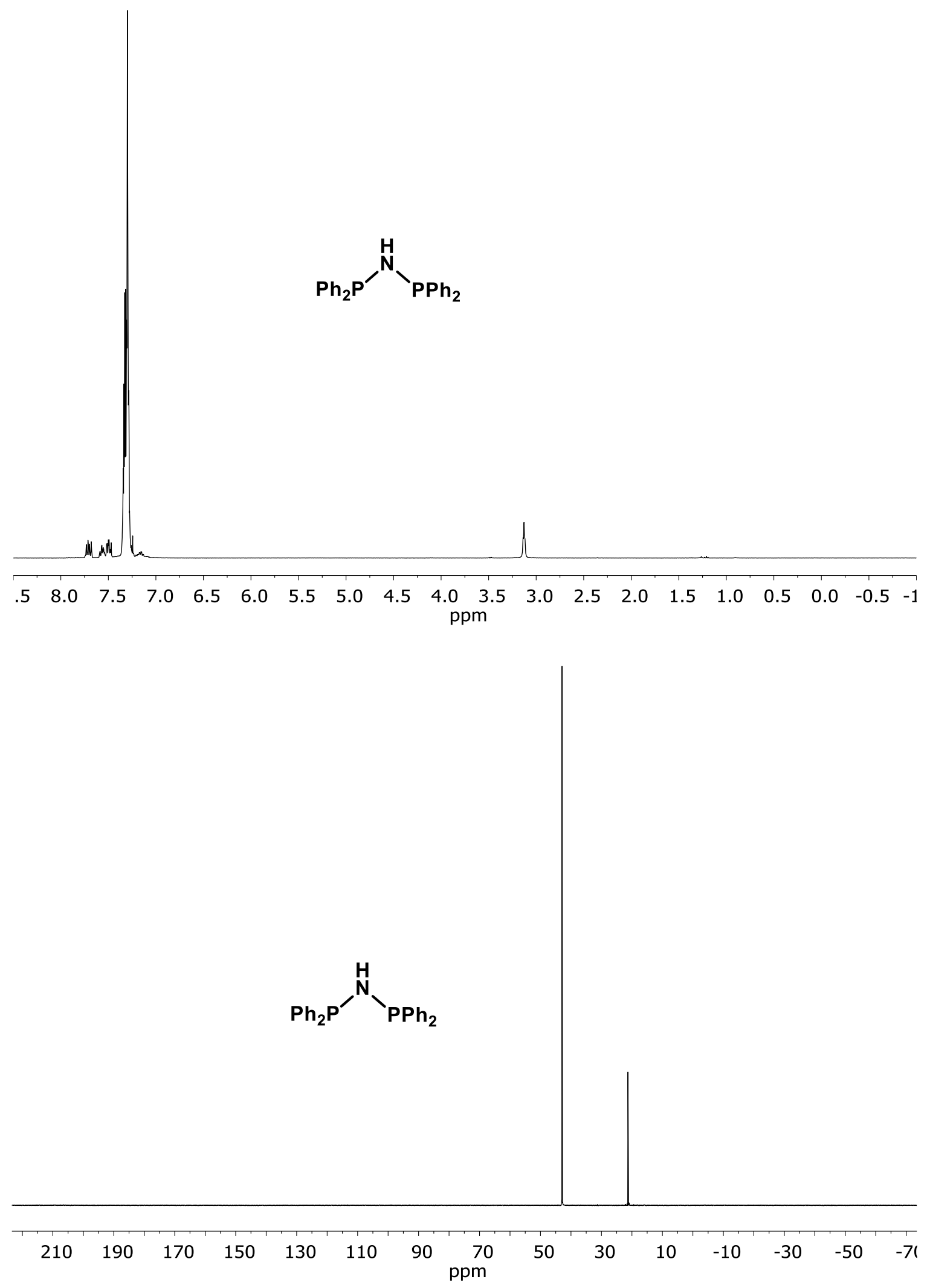
Figure S26. ${ }^{1} \mathrm{H}$ and ${ }^{31} \mathrm{P}$ NMR spectra of ligand 29 in $\mathrm{C}_{6} \mathrm{D}_{6} ; N$-(diphenylphosphino)- $P, P$-diphenyl- $N$-2pyridinyl-phosphinous amide.
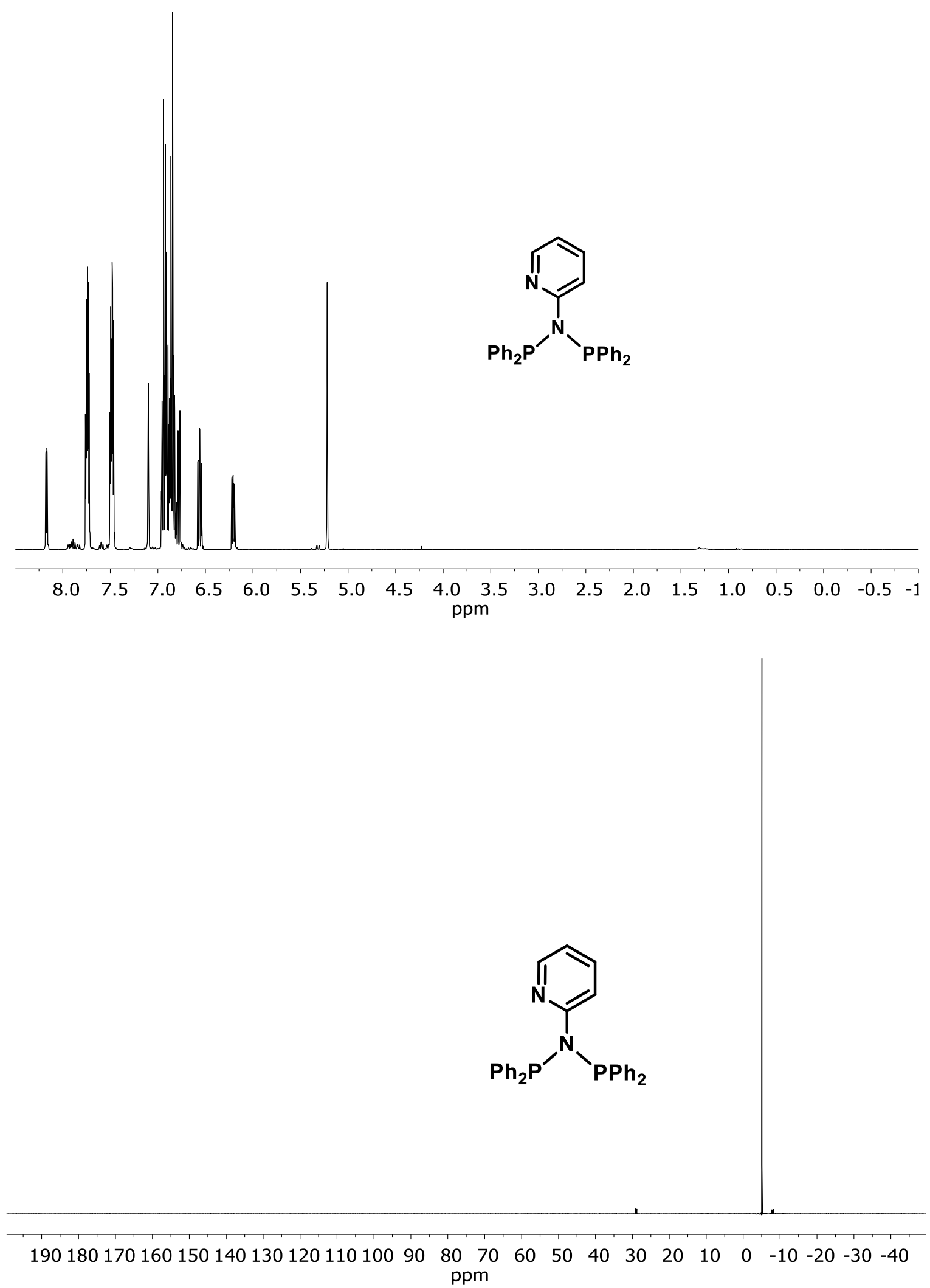
Figure S27. ${ }^{1} \mathrm{H}$ and ${ }^{31} \mathrm{P}$ NMR spectra of ligand 30 in $\mathrm{CDCl}_{3} ; 1,1$ '-(1E)-1,2-ethenediylbis[1,1-diphenylphosphine].
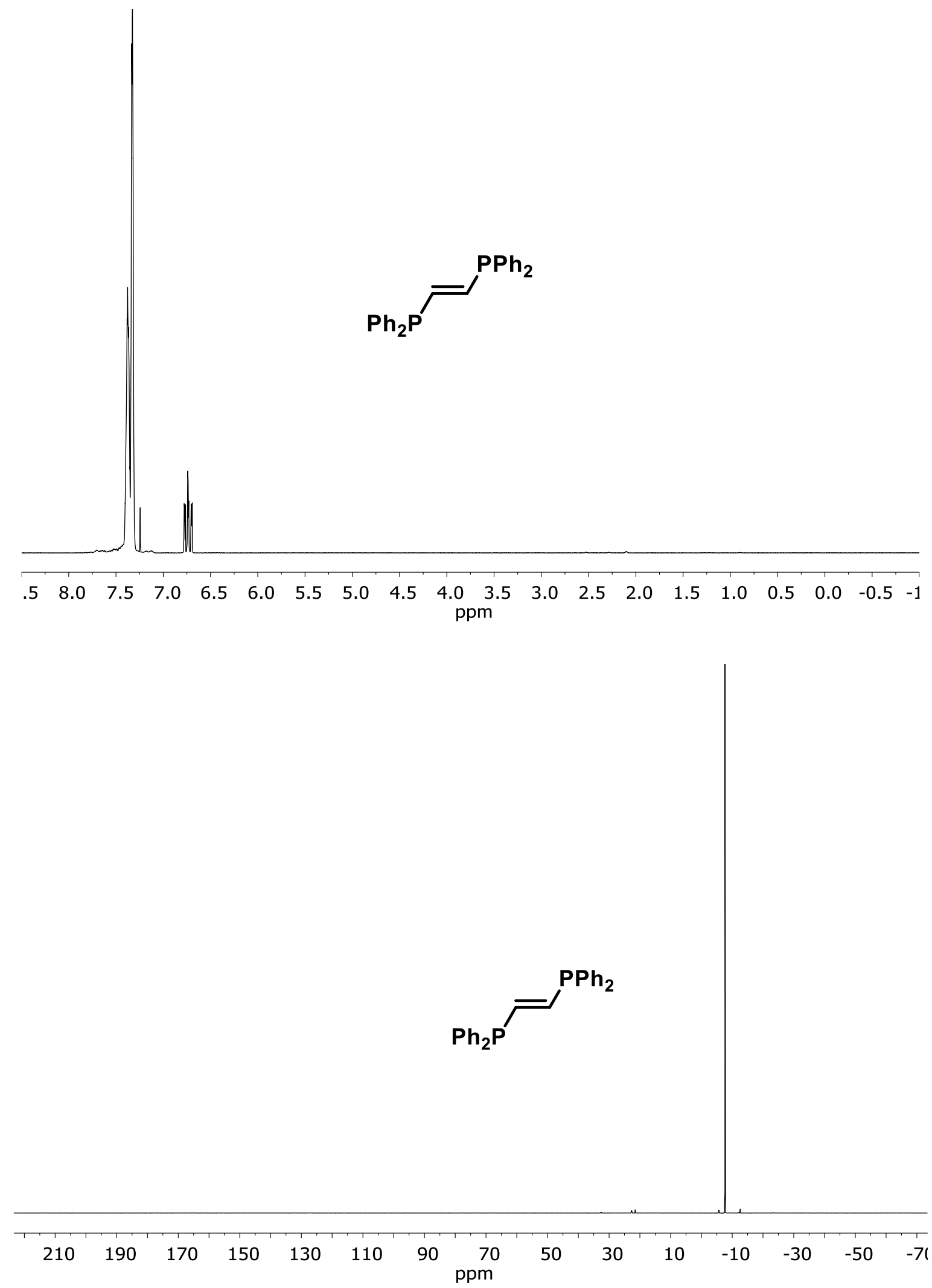
Figure S28. ${ }^{1} \mathrm{H}$ and ${ }^{31} \mathrm{P}$ NMR spectra of ligand 31 in $\mathrm{C}_{6} \mathrm{D}_{6} ; 1,11^{\prime}-[(1 S)$-1-methyl-1,2-ethanediyl]bis[1,1diphenylphosphine].
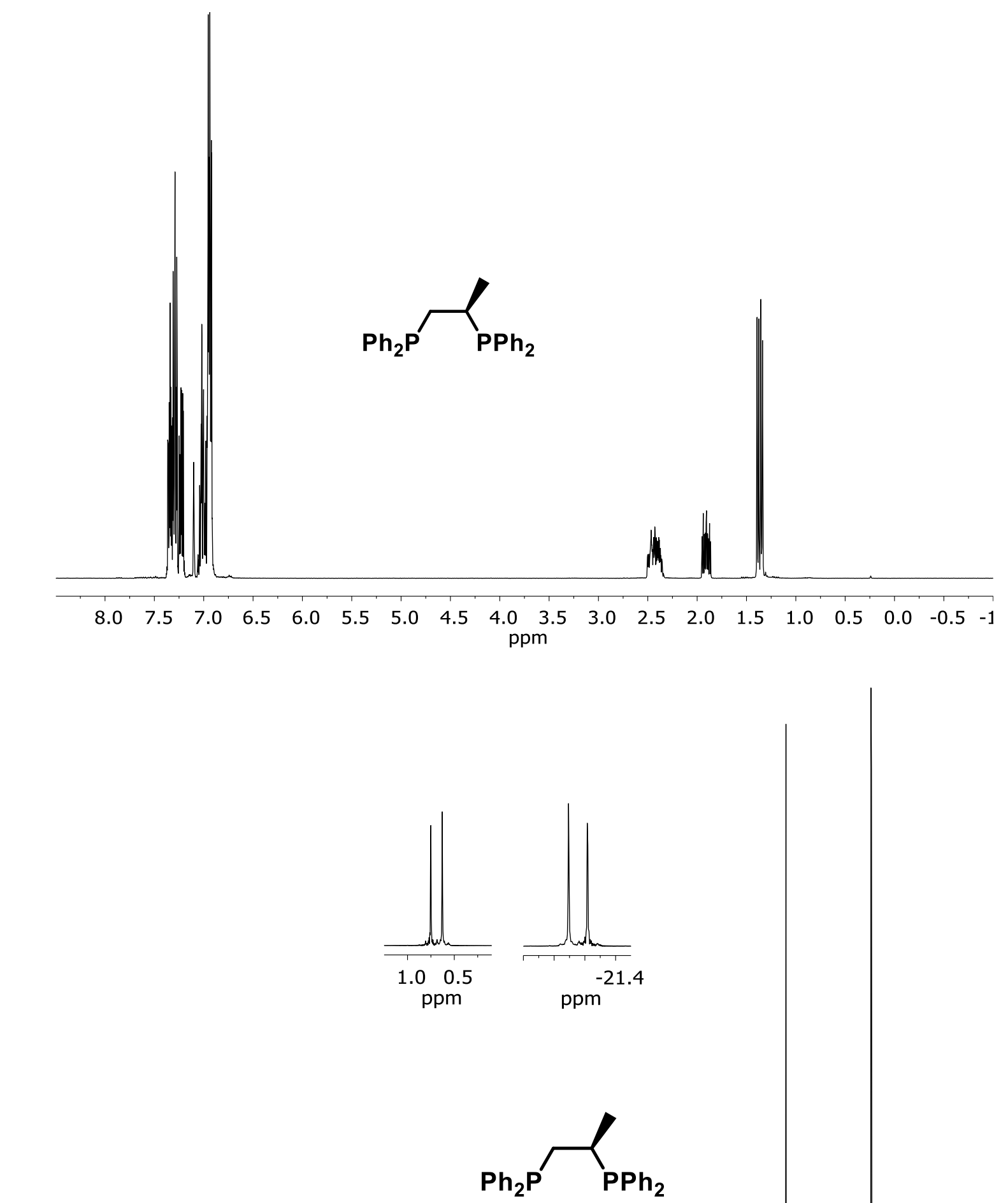
Figure S29. ${ }^{1} \mathrm{H}$ and ${ }^{31} \mathrm{P}$ NMR spectra of ligand 32 in $\mathrm{C}_{6} \mathrm{D}_{6} ;\left(\boldsymbol{R}, \boldsymbol{R}^{\prime}\right)$-Chiraphos, 1,1'-[(1R,2R)-1,2-dimethyl1,2-ethanediyl]bis[1,1-diphenylphosphine].
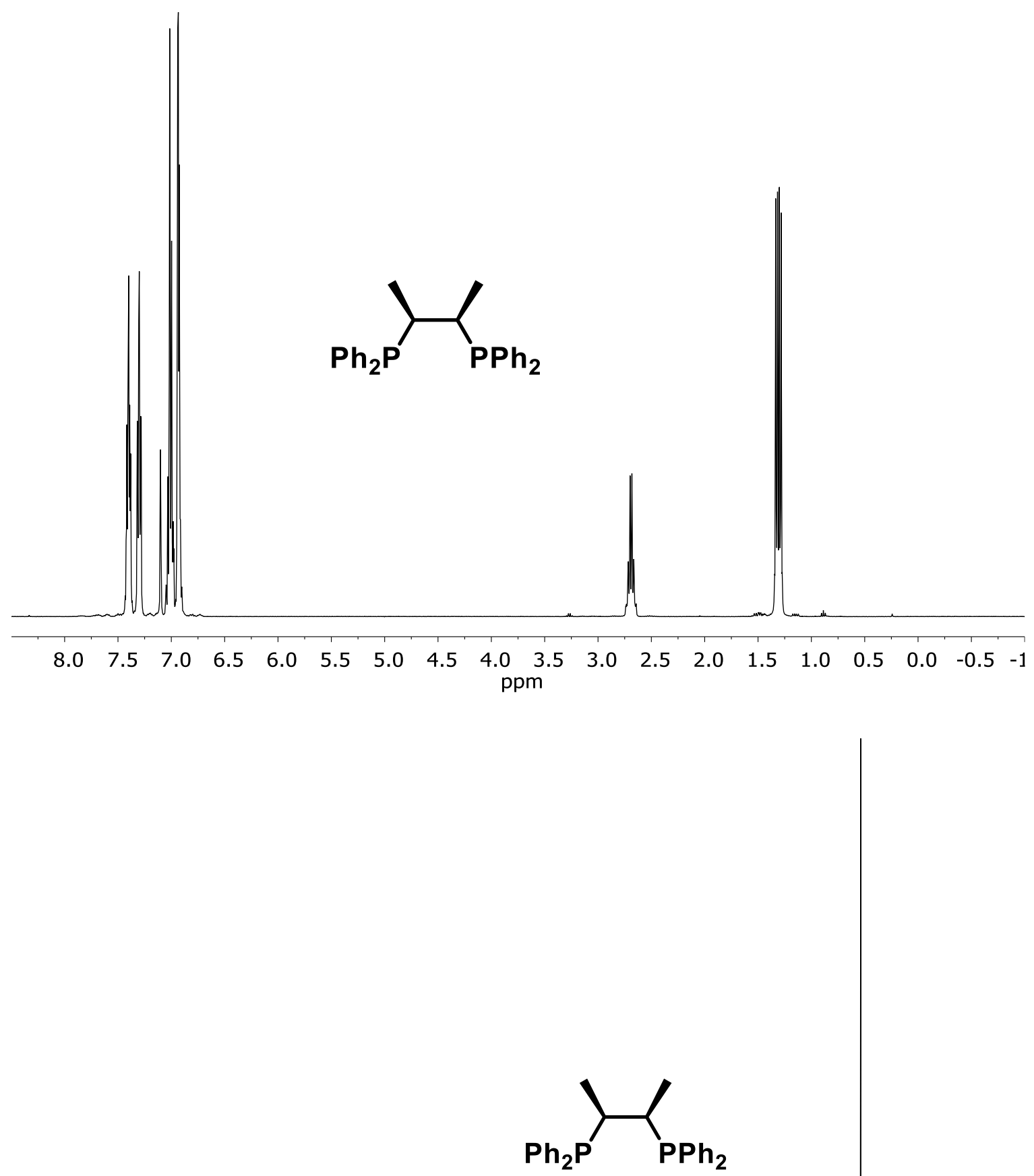

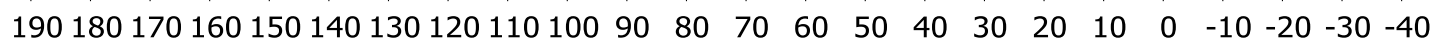
ppm 
Figure S30. ${ }^{1} \mathrm{H}$ and ${ }^{31} \mathrm{P}$ NMR spectra of ligand 33 in $\mathrm{CDCl}_{3} ; 1,1$ '-[[1,1'-biphenyl]-2,2'-diyl]bis[1,1diphenylphosphine].
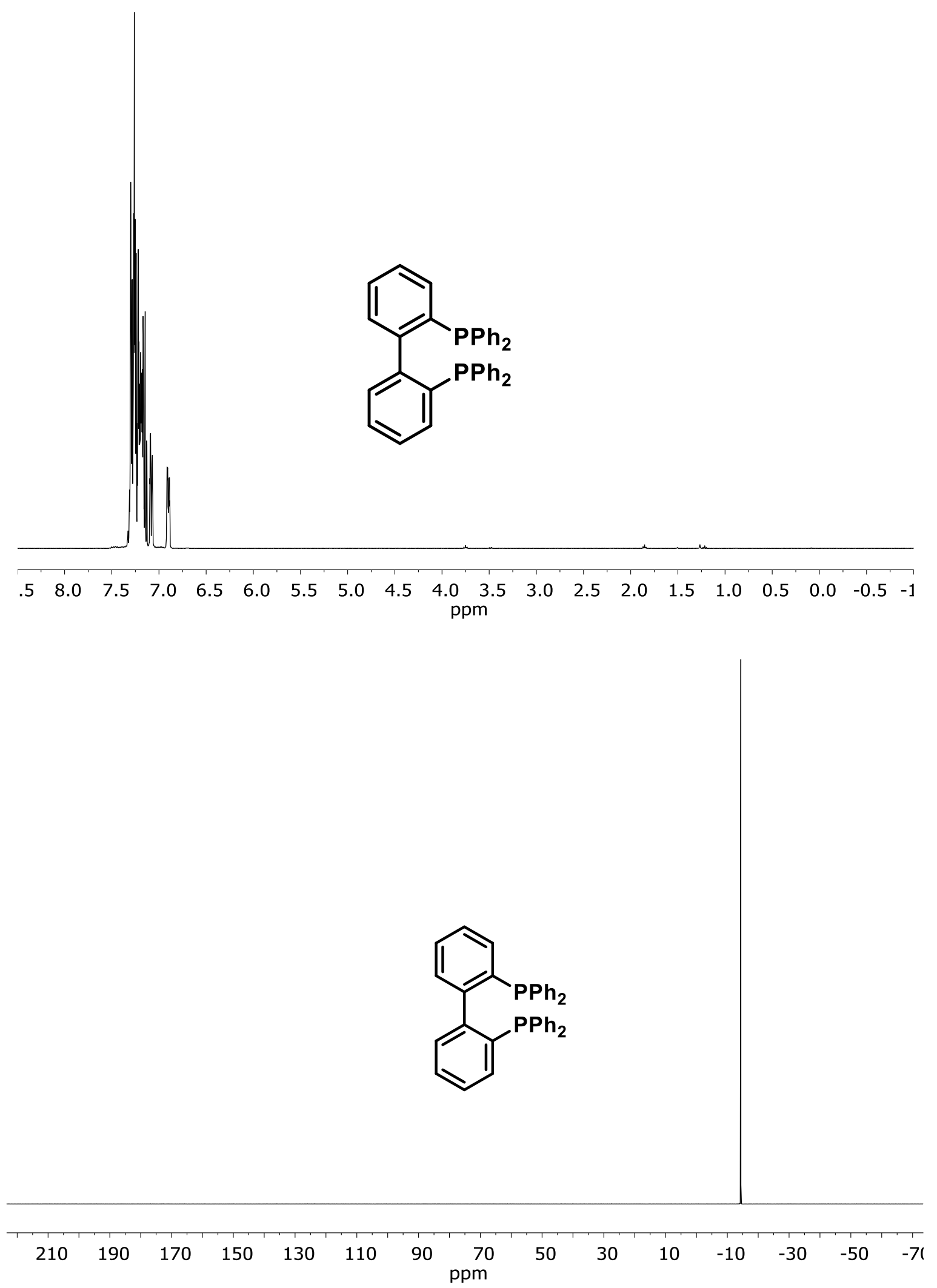
Figure S31. ${ }^{1} \mathrm{H}$ and ${ }^{31} \mathrm{P}$ NMR spectra of ligand 34 in $\mathrm{C}_{6} \mathrm{D}_{6} ; 1,1$ '-[[1,1'-biphenyl]-2,2'-diylbis(methylene)] bis[1,1-diphenylphosphine].
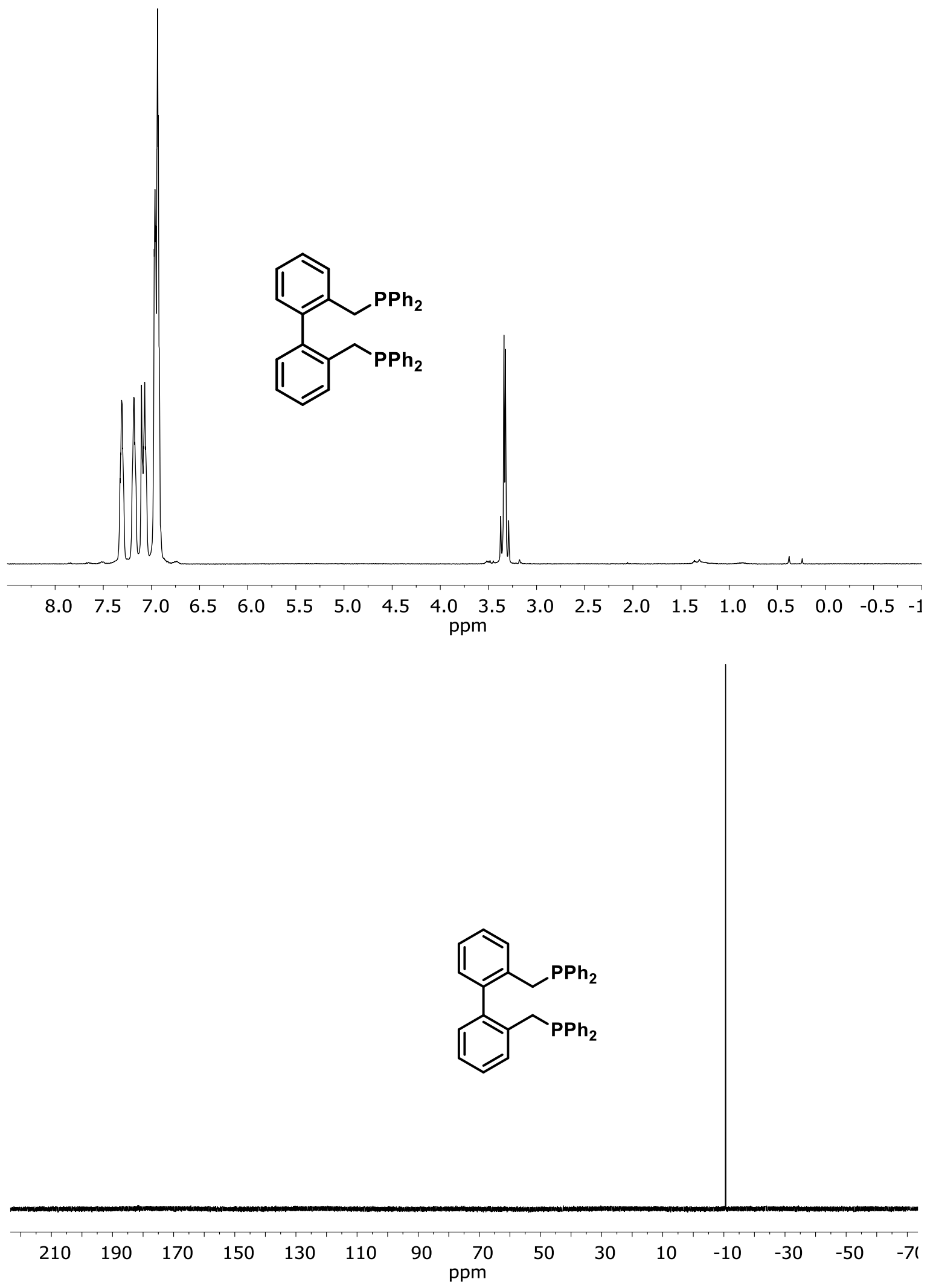
Figure S32. ${ }^{1} \mathrm{H}$ and ${ }^{31} \mathrm{P}$ NMR spectra of ligand 35 in $\mathrm{C}_{6} \mathrm{D}_{6} ; 1,1$ '-(1R)-[1,1'-binaphthalene]-2,2'diylbis[bis(4-methylphenyl)phosphine].
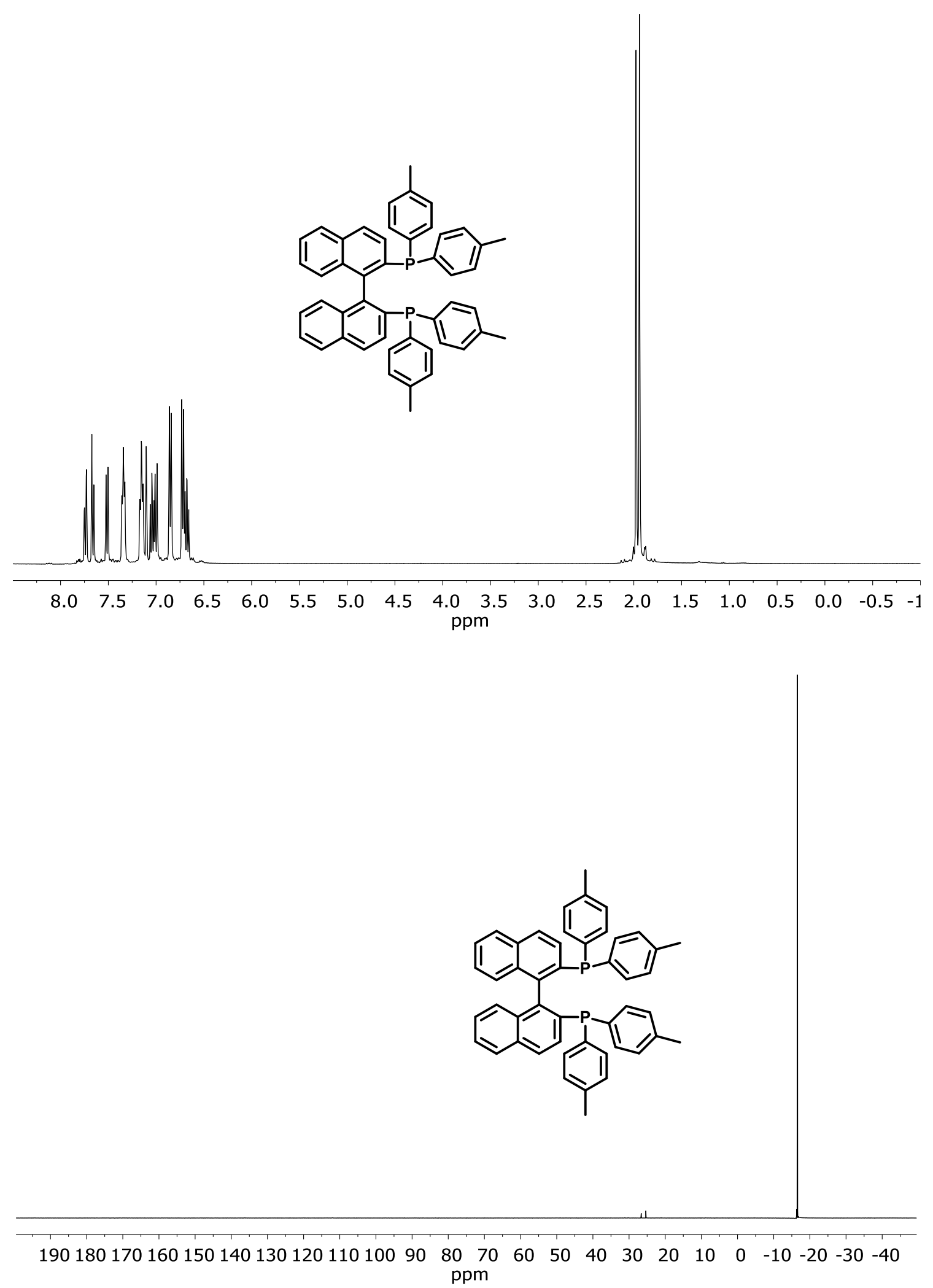
Figure S33. ${ }^{1} \mathrm{H}$ and ${ }^{31} \mathrm{P}$ NMR spectra of ligand 36 in $\mathrm{C}_{6} \mathrm{D}_{6}$; 1, 1'-(1,1',3,3'-tetrahydro[5,5'-biisobenzofuran]6,6'-diyl)bis[1,1-diphenylphosphine].
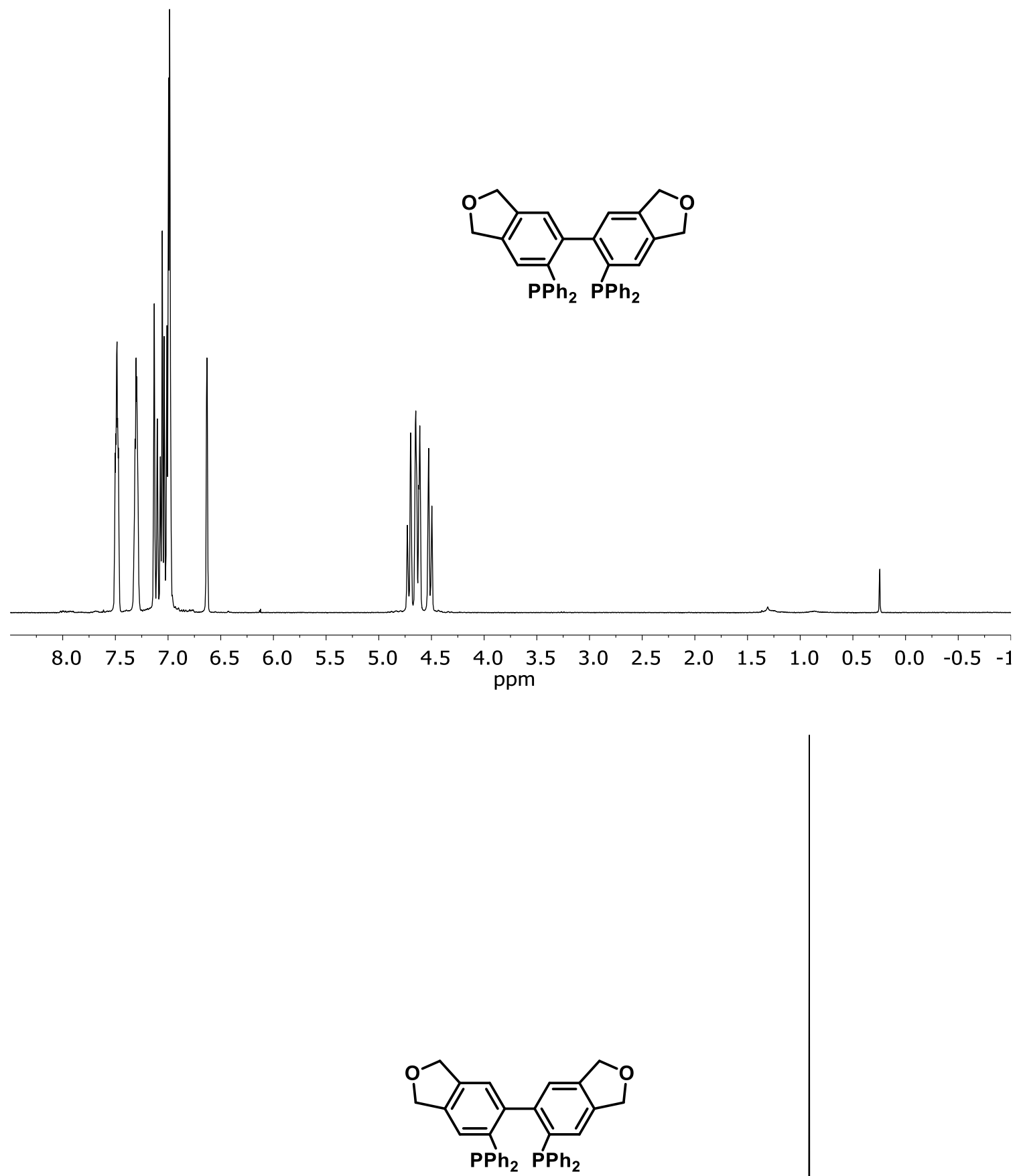

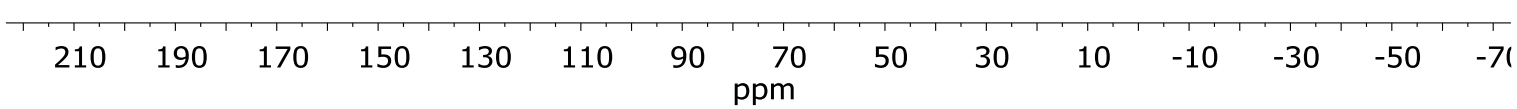




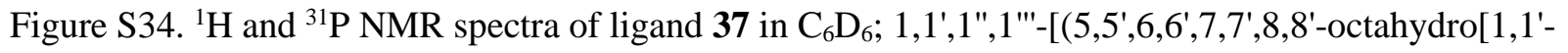
binaphthalene]-2,2'-diyl)bis(oxyphosphinidyne)]tetrakis- $H$-indole.
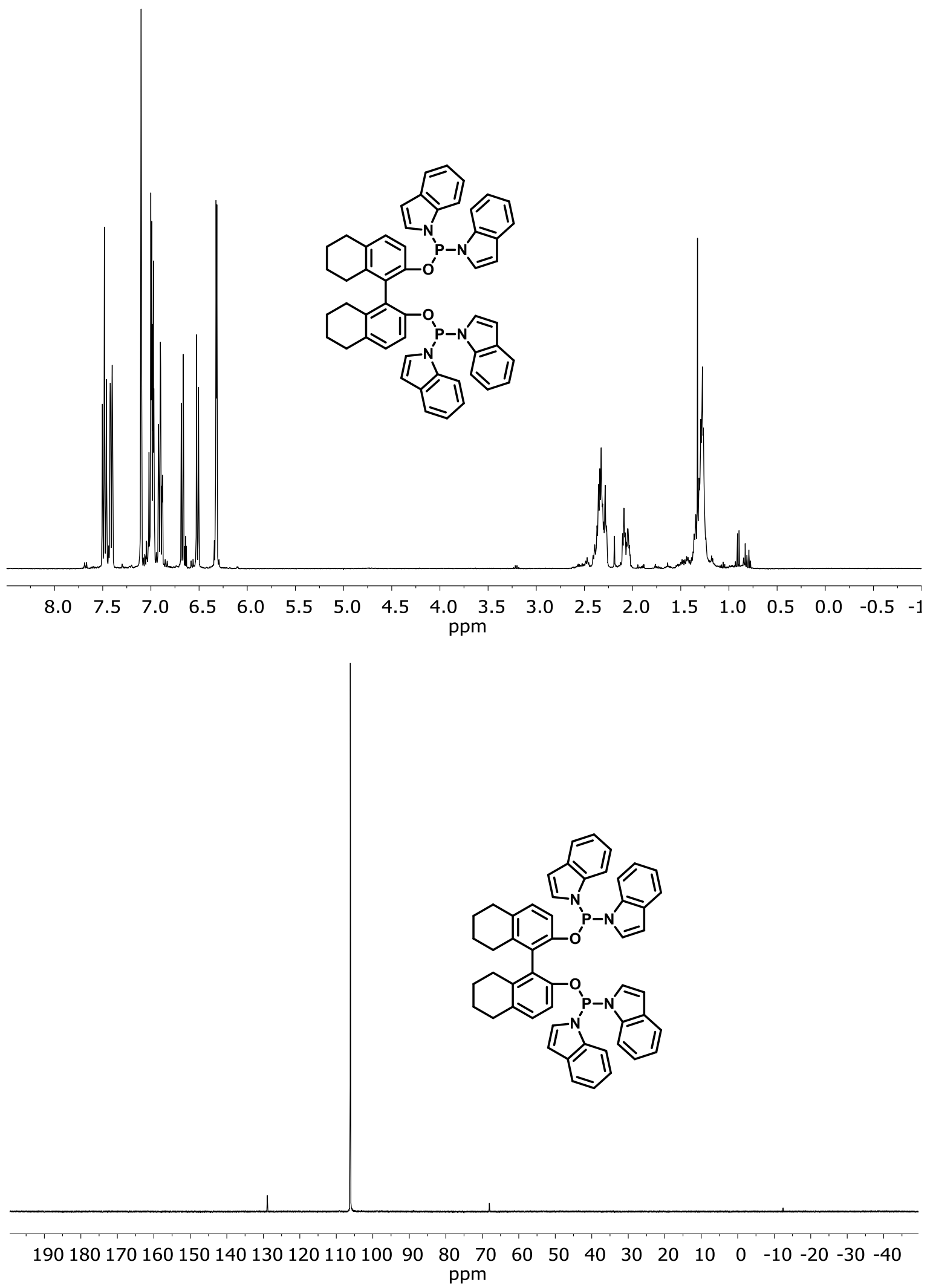
Figure S35. ${ }^{1} \mathrm{H}$ and ${ }^{31} \mathrm{P}$ NMR spectra of ligand 38 in $\mathrm{C}_{6} \mathrm{D}_{6} ; S$-(-)-2,2'-bis(N-diphenylphosphinoamino)5,5',6,6',7,7',8,8'-octahydro-1,1'-binaphthyl.
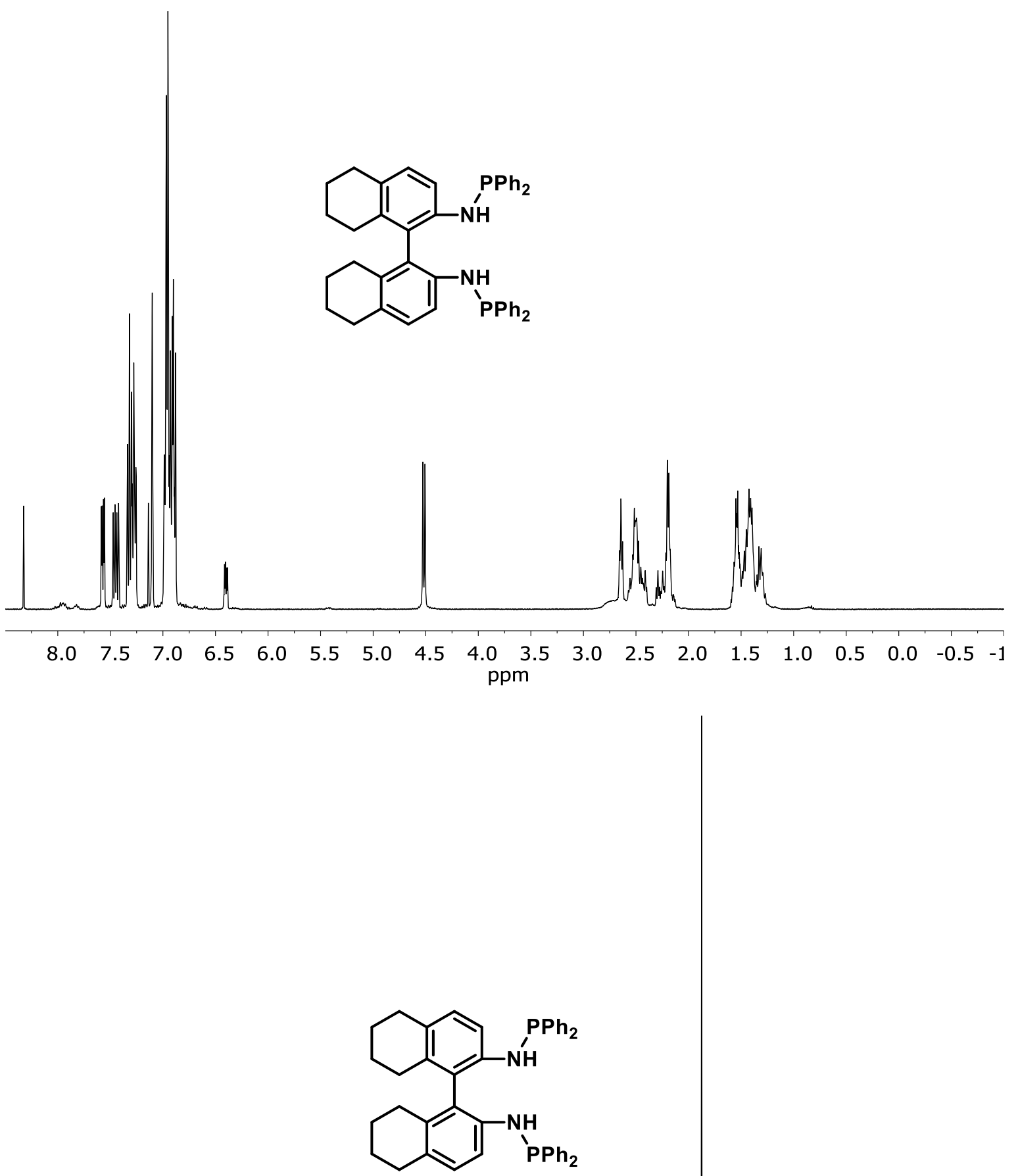
Figure S36. ${ }^{1} \mathrm{H}$ and ${ }^{31} \mathrm{P}$ NMR spectra of ligand 39 in $\mathrm{CDCl}_{3}$; (S)-Phanephos, 1,1'-tricyclo[8.2.2.2 $\left.{ }^{4,7}\right]$ hexadeca-4,6,10,12,13,15-hexaene-5,11-diylbis[1,1-diphenylphosphine].
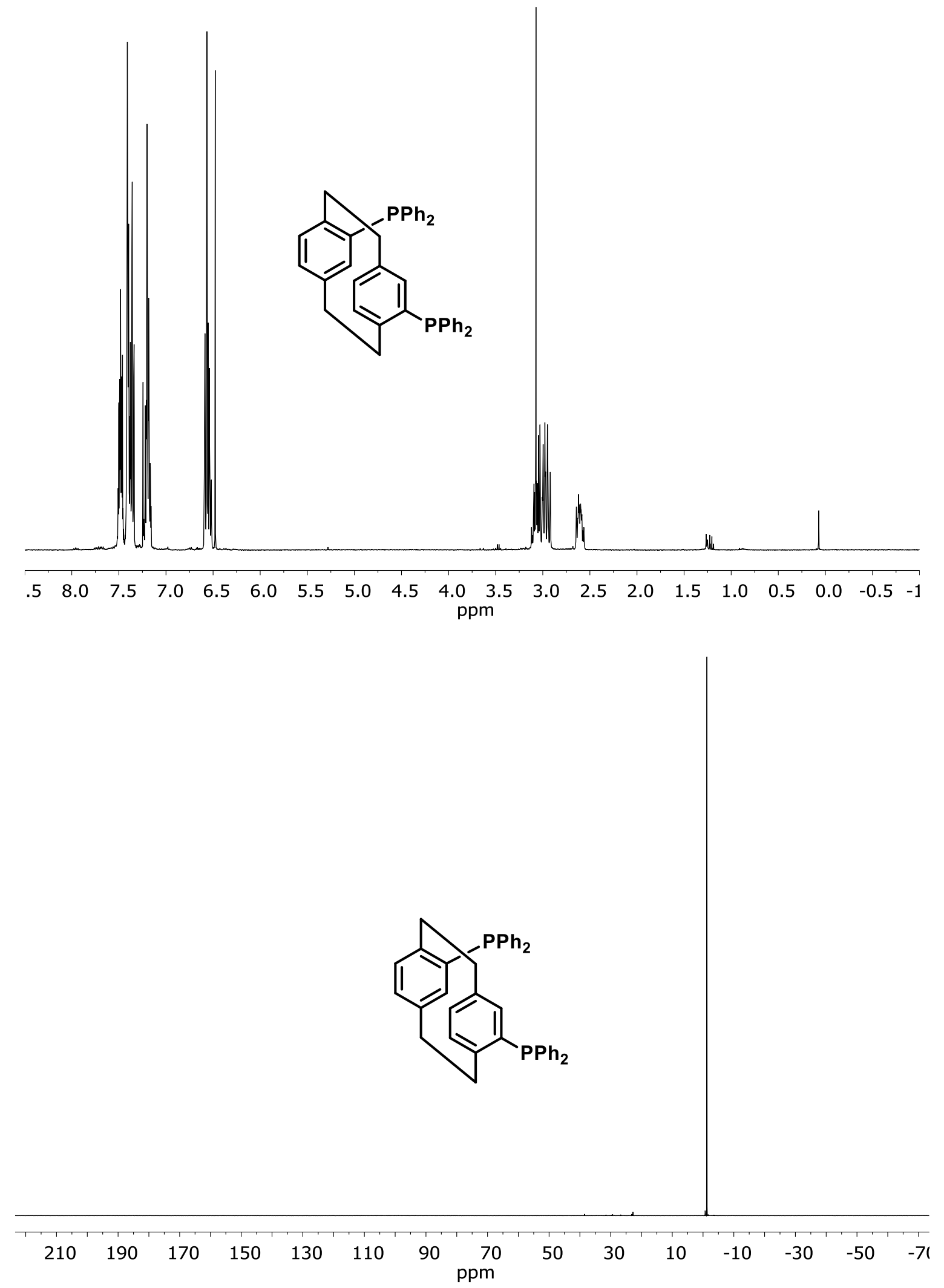
Figure S37. ${ }^{1} \mathrm{H}$ and ${ }^{31} \mathrm{P}$ NMR spectra of ligand 40 in $\mathrm{CDCl}_{3}$.
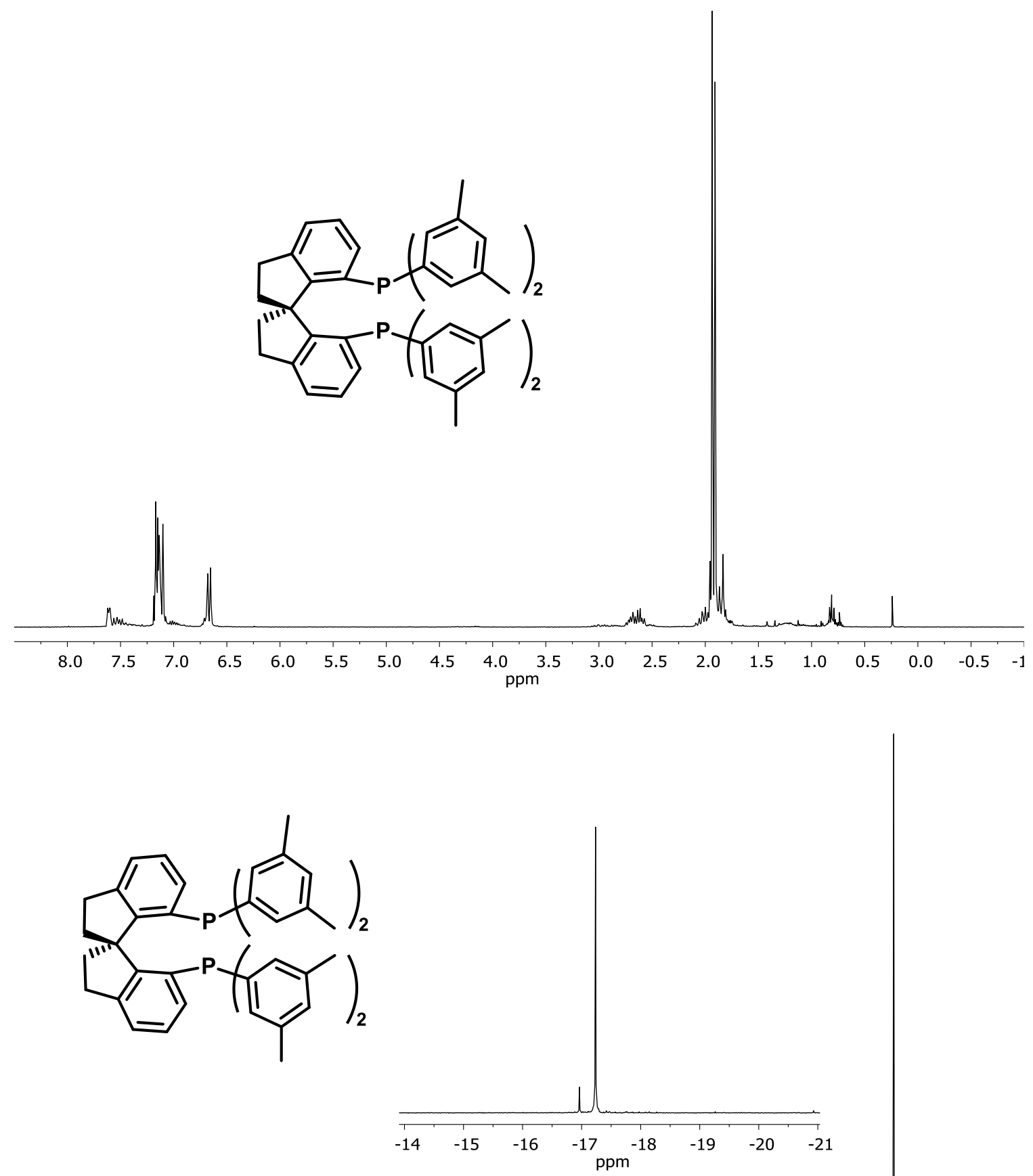

\begin{tabular}{llllllllllllllllllllllllll}
\hline 00 & 190 & 180 & 170 & 160 & 150 & 140 & 130 & 120 & 110 & 100 & 90 & 80 & 70 & 60 & 50 & 40 & 30 & 20 & 10 & 0 & -10 & -20 & -30 & -40 & $-!$
\end{tabular} 
Figure S38. ${ }^{1} \mathrm{H}$ and ${ }^{31} \mathrm{P}$ NMR spectra of ligand 41 in $\mathrm{C}_{6} \mathrm{D}_{6}$; $(2 R, 3 R)-(-)-2,3$-bis(diphenylphosphino)bicyclo[2.2.1]hept-5-ene.
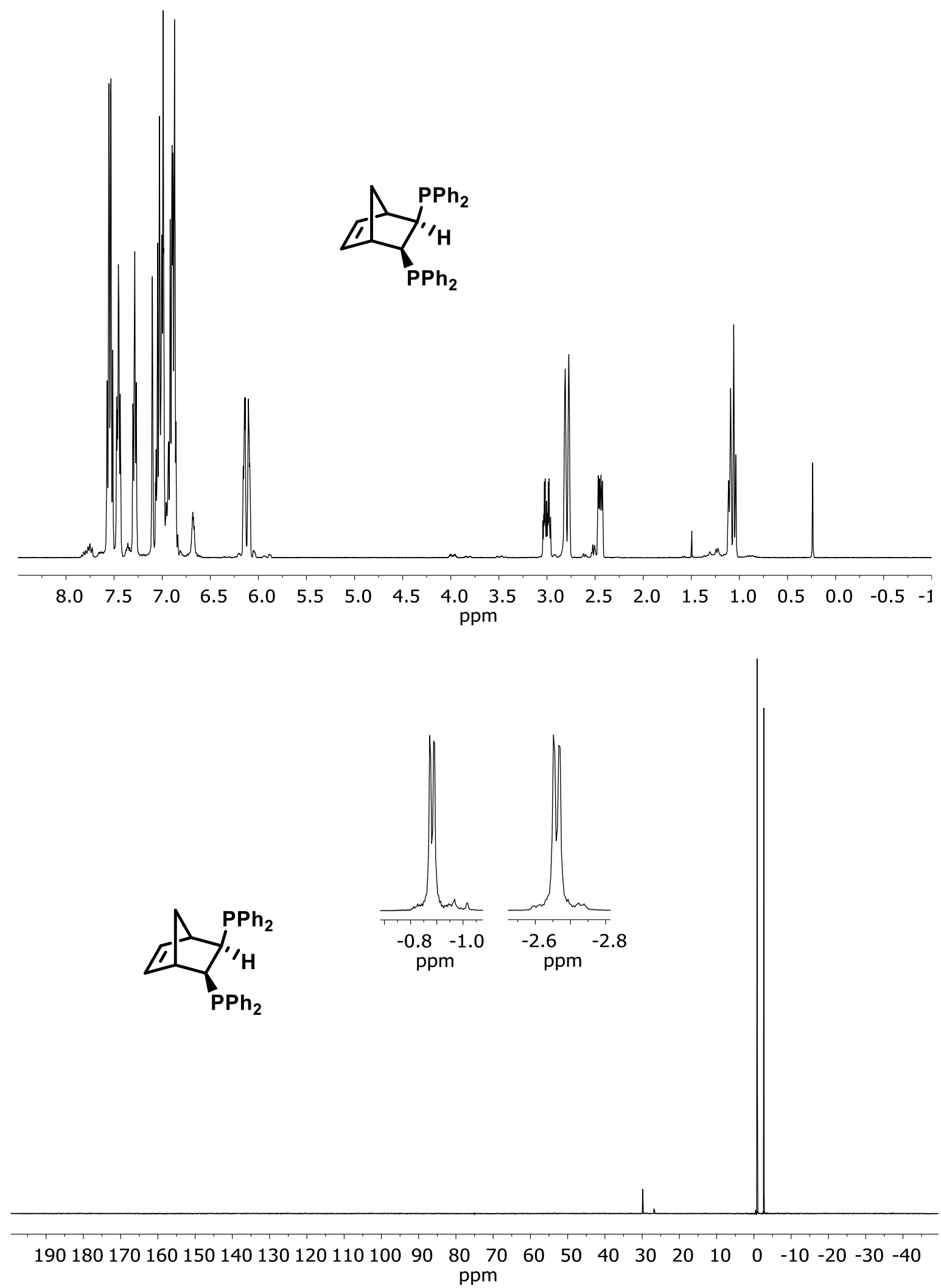
Figure S39. ${ }^{1} \mathrm{H}$ and ${ }^{31} \mathrm{P}$ NMR spectra of ligand 42 in $\mathrm{C}_{6} \mathrm{D}_{6}$; [(1S,4R)-3-[4-(diphenylphosphino)-2,5dimethyl-3-thienyl]-4,7,7-trimethylbicyclo[2.2.1]hept-2-en-2-yl]diphenylphosphine.
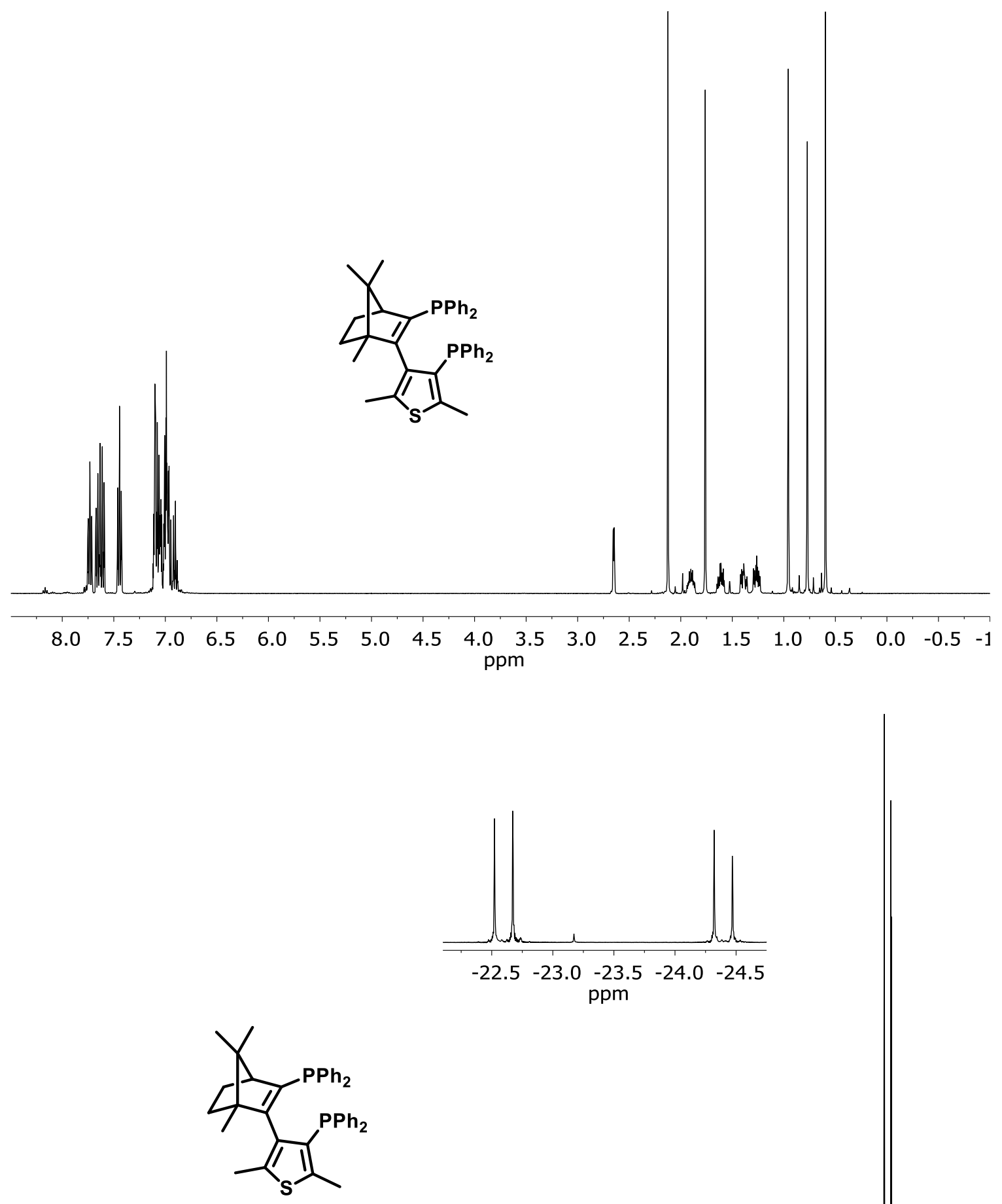
Figure S40. ${ }^{1} \mathrm{H}$ and ${ }^{31} \mathrm{P}$ NMR spectra of ligand 43 in $\mathrm{C}_{6} \mathrm{D}_{6} ;(2 R, 4 R)-4$-(diphenylphosphino)-2[(diphenylphosphino)methyl]pyrrolidine.
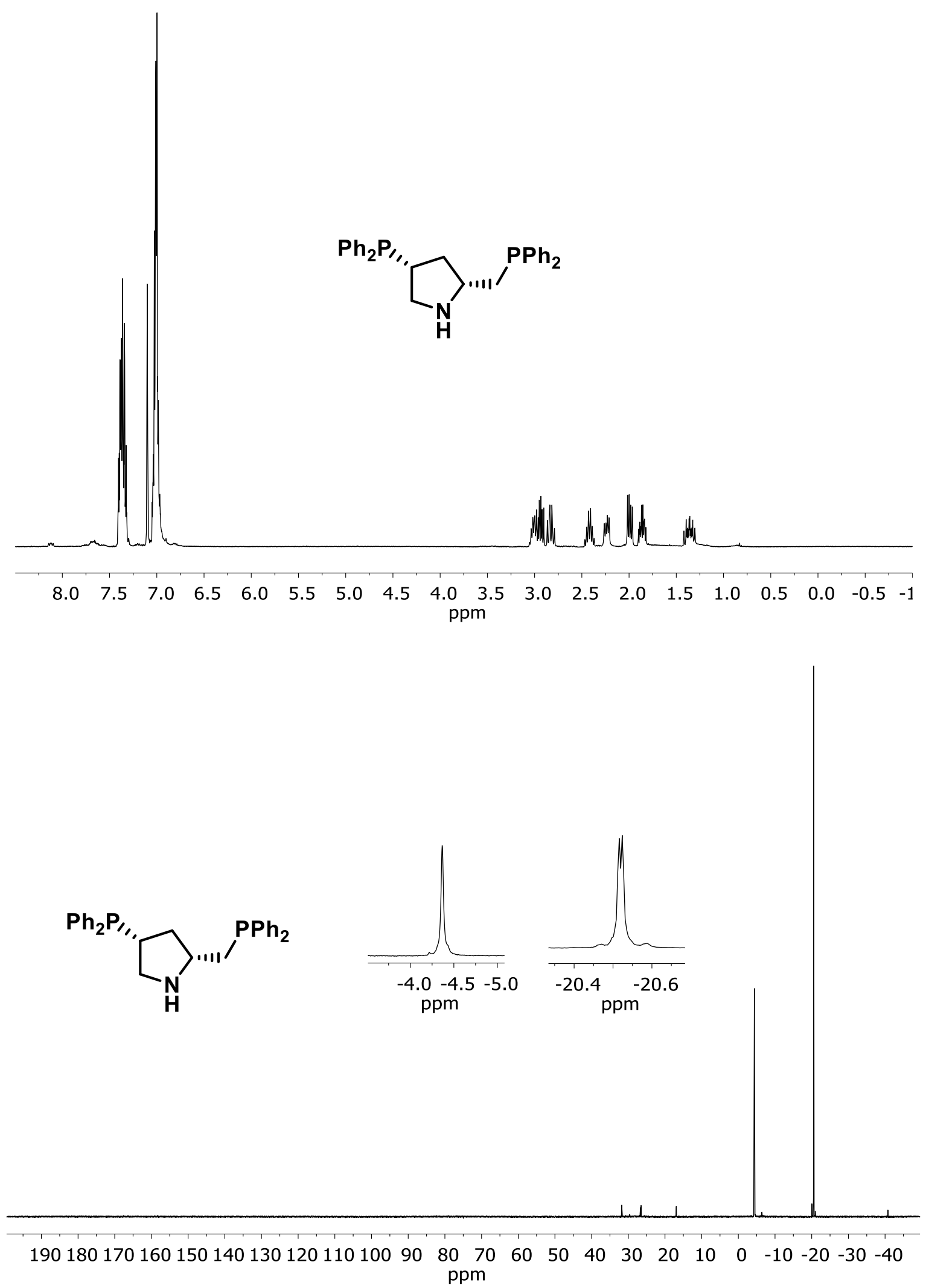
Figure S41. ${ }^{1} \mathrm{H}$ and ${ }^{31} \mathrm{P}$ NMR spectra of ligand 44 in $\mathrm{C}_{6} \mathrm{D}_{6}$; (2S,4S)-(-)-2-(diphenylphosphinomethyl)-4(diphenylphosphino)-N-(t-butoxycarbonyl)pyrrolidine.
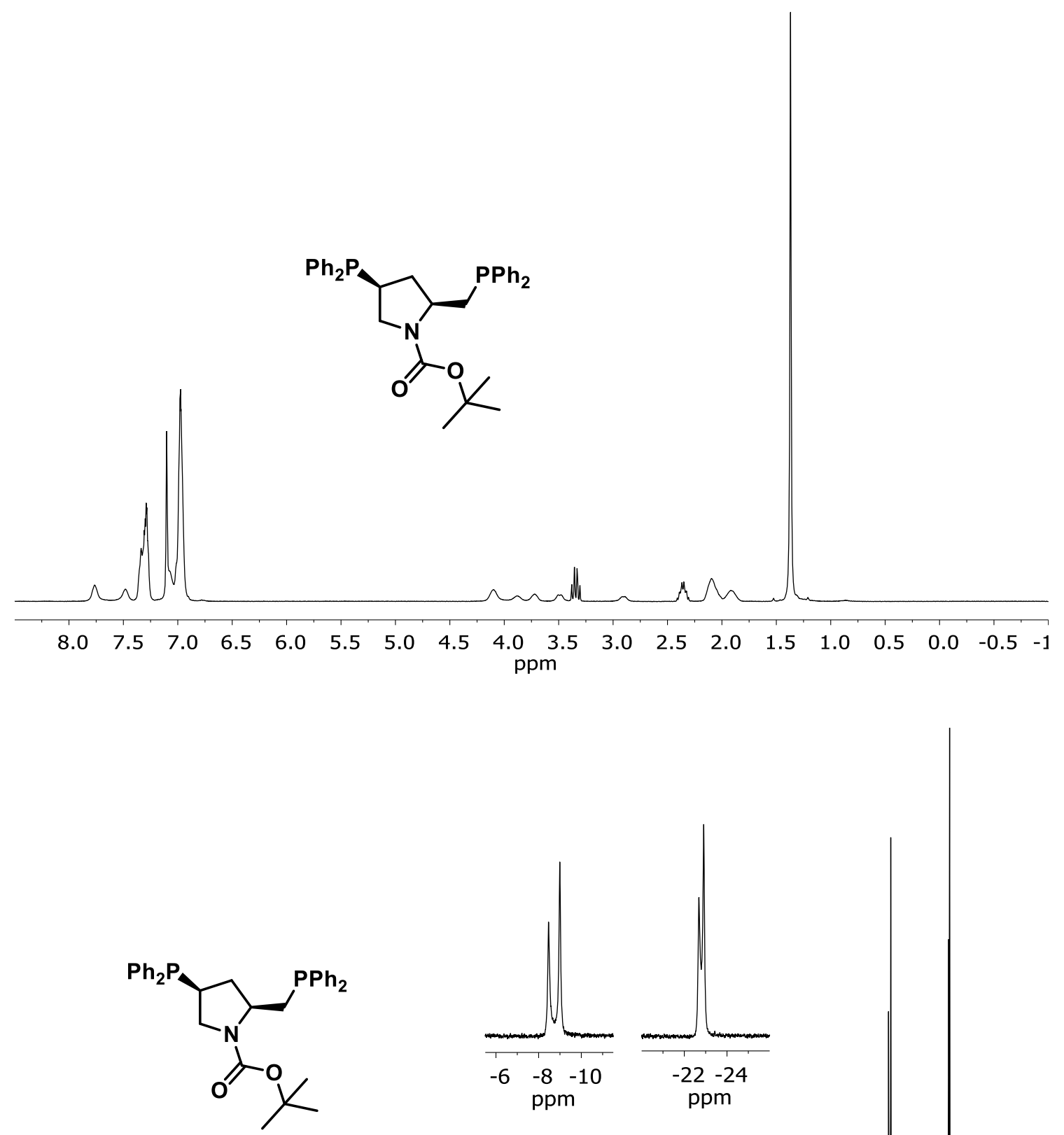

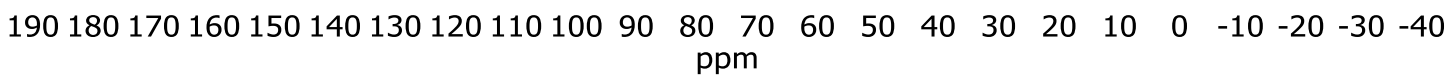


Figure S42. ${ }^{1} \mathrm{H}$ and ${ }^{31} \mathrm{P}$ NMR spectra of ligand 45 in $\mathrm{C}_{6} \mathrm{D}_{6}$; 1,1'-[[(4R,5R)-2,2-dimethyl-1,3-dioxolane-4,5diyl]bis(methylene)]bis[1,1-diphenylphosphine].
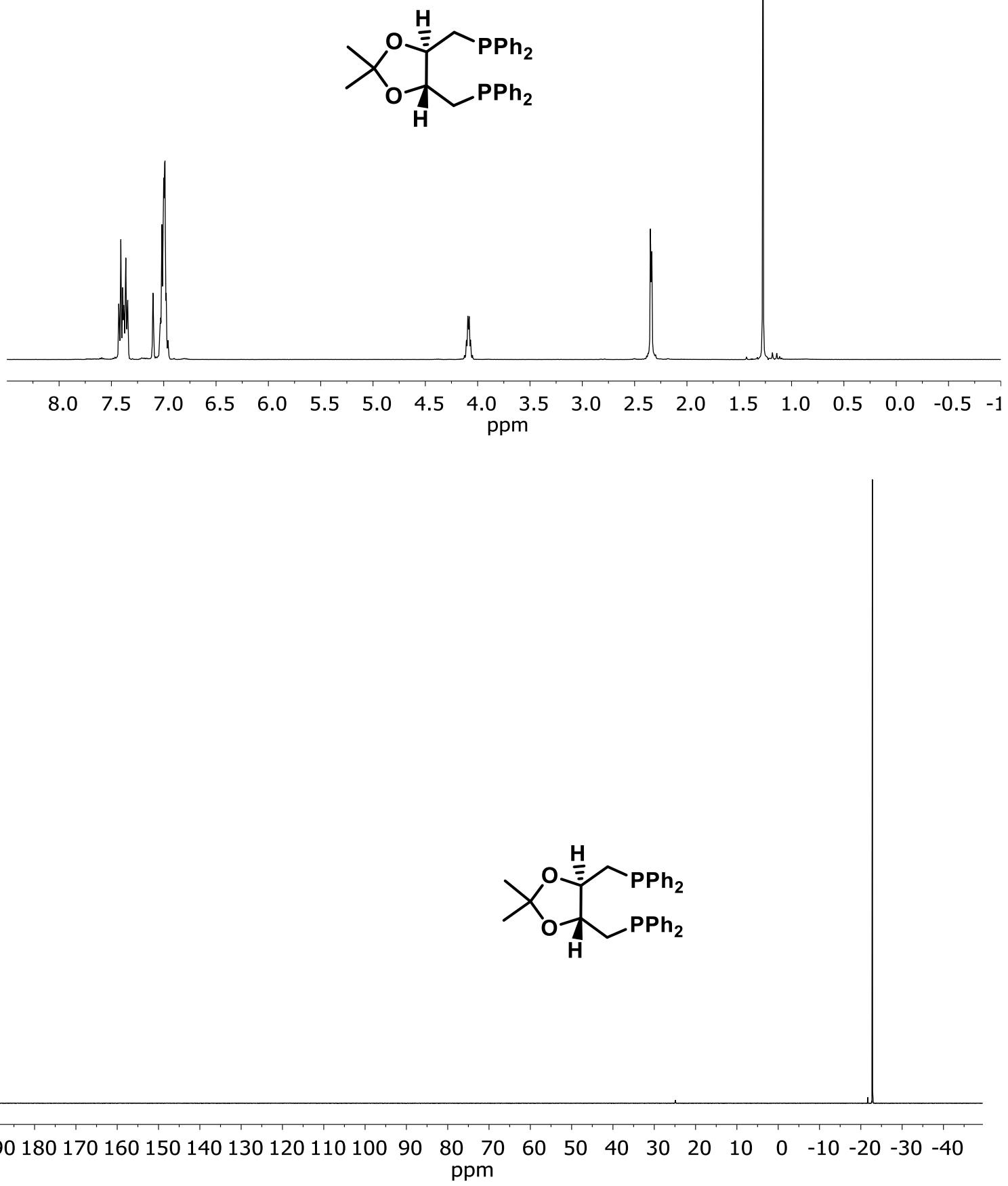
Figure S43. ${ }^{1} \mathrm{H}$ and ${ }^{31} \mathrm{P}$ NMR spectrum of ligand 46 in $\mathrm{C}_{6} \mathrm{D}_{6}$. (5aR,8aR)-1,13-Bis(di-p-tolylphosphanyl)5a,6,7,8,8a,9-hexahydro-5H-chromeno[3,2-d]xanthene.
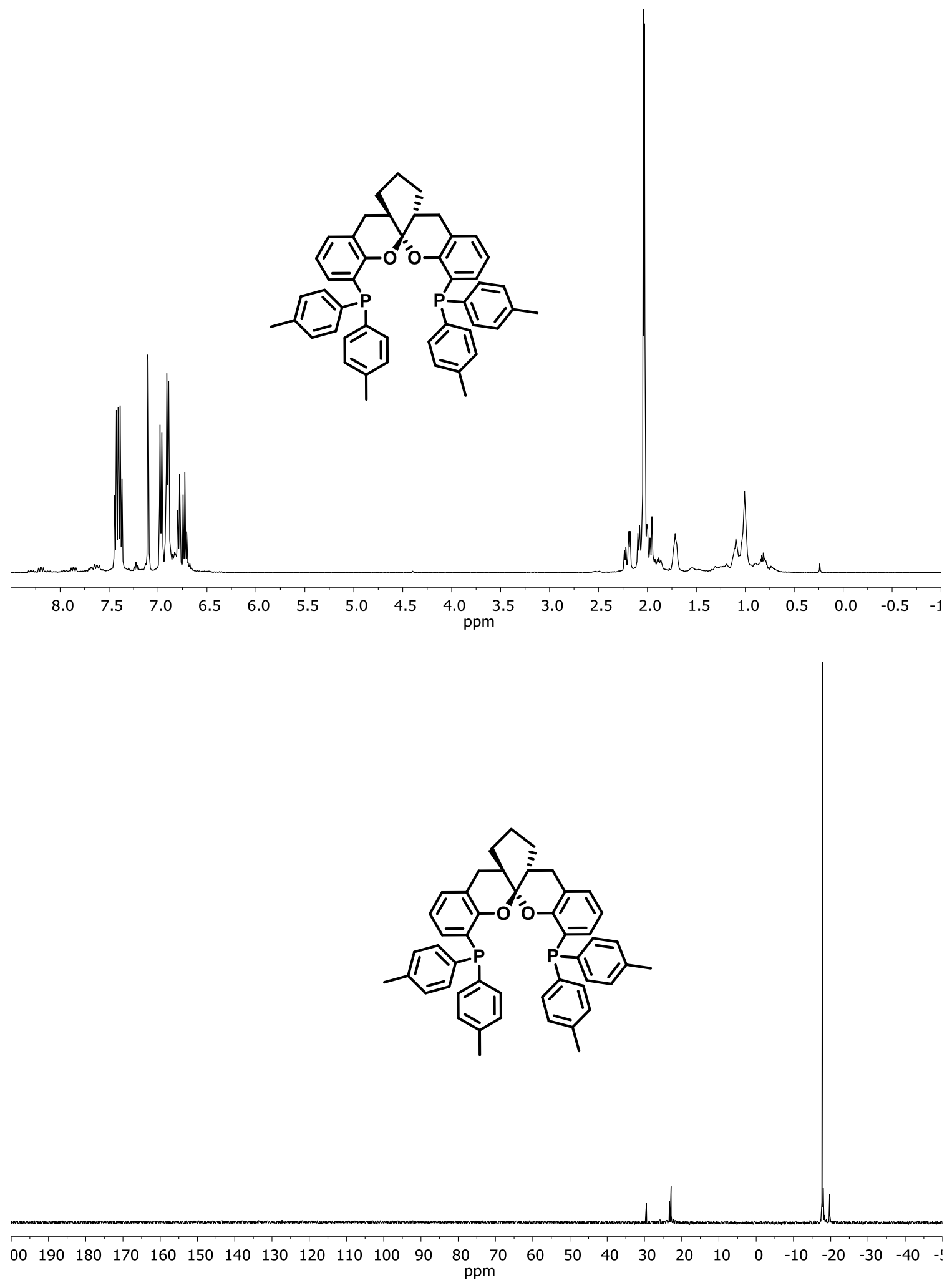
Figure S44. ${ }^{31} \mathrm{P}$ NMR spectrum of ligand 47 in $\mathrm{C}_{6} \mathrm{D}_{6} ;$ rac-trans-1,2-bis[di(3,5-dimethylphenyl) phosphinomethyl]cyclobutane.
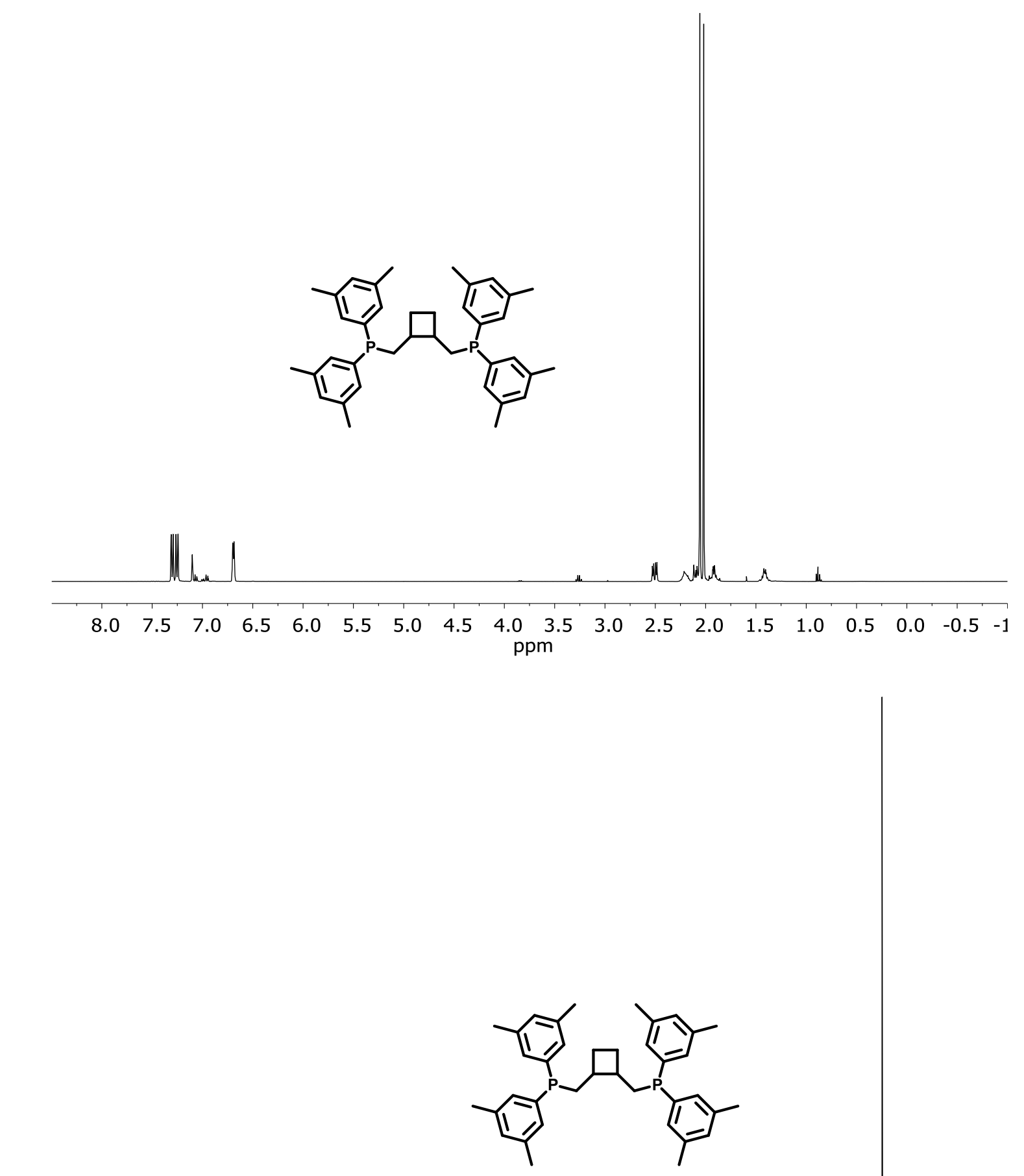

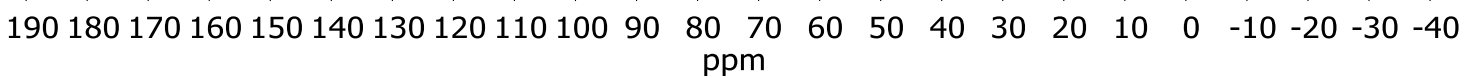


Figure S45. ${ }^{1} \mathrm{H}$ and ${ }^{31} \mathrm{P}$ NMR spectra of ligand 48 in $\mathrm{C}_{6} \mathrm{D}_{6}$; dppf, 1,1'-bis(diphenylphosphino)ferrocene.
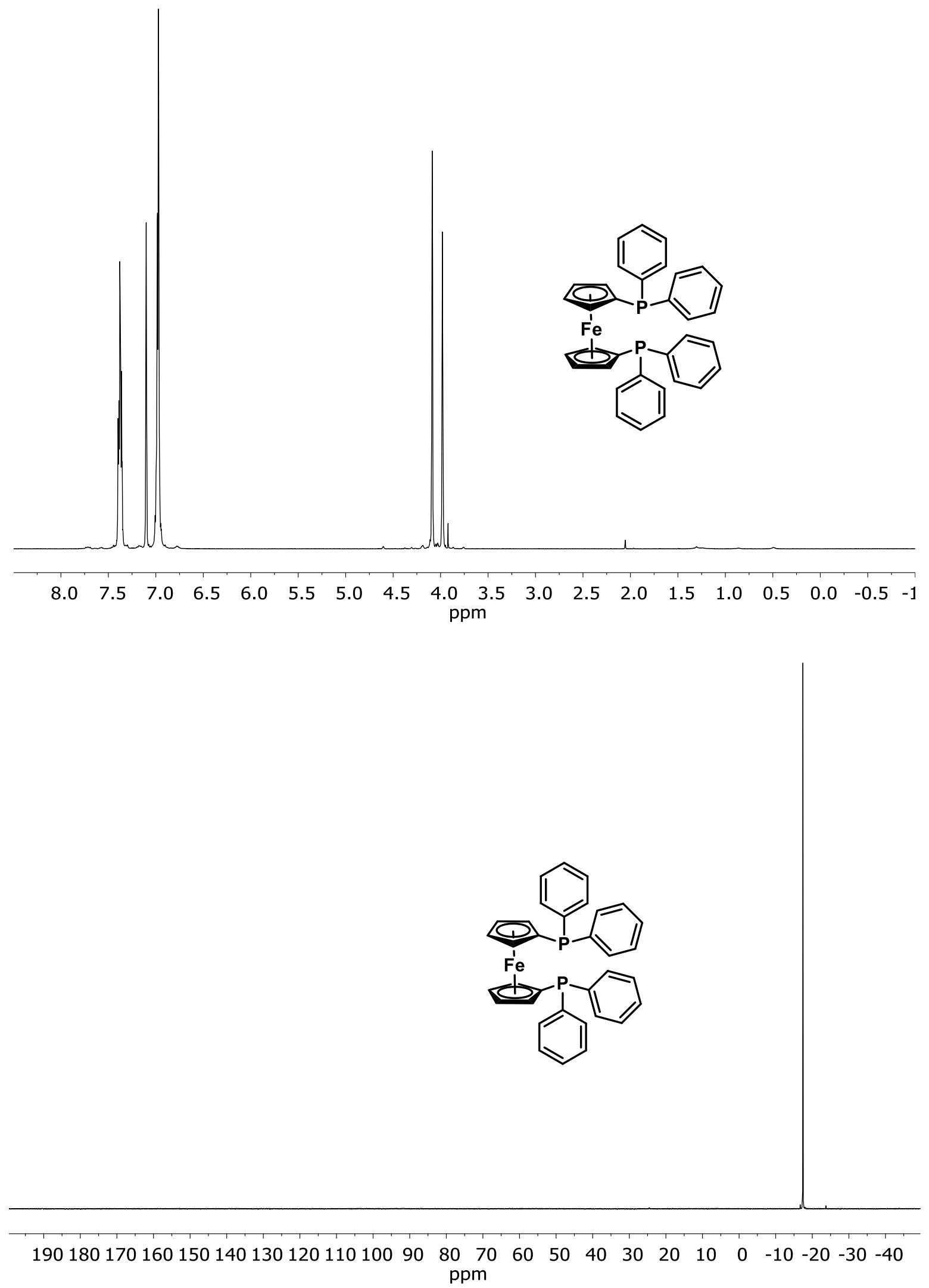
Figure S46. ${ }^{1} \mathrm{H}$ and ${ }^{31} \mathrm{P}$ NMR spectra of ligand 49 in $\mathrm{C}_{6} \mathrm{D}_{6}$; $(S)-(-)-[(S)$-2-diphenylphosphinoferrocenyl] ( $N, N$-dimethylamino)(2-diphenylphosphinophenyl)methane.
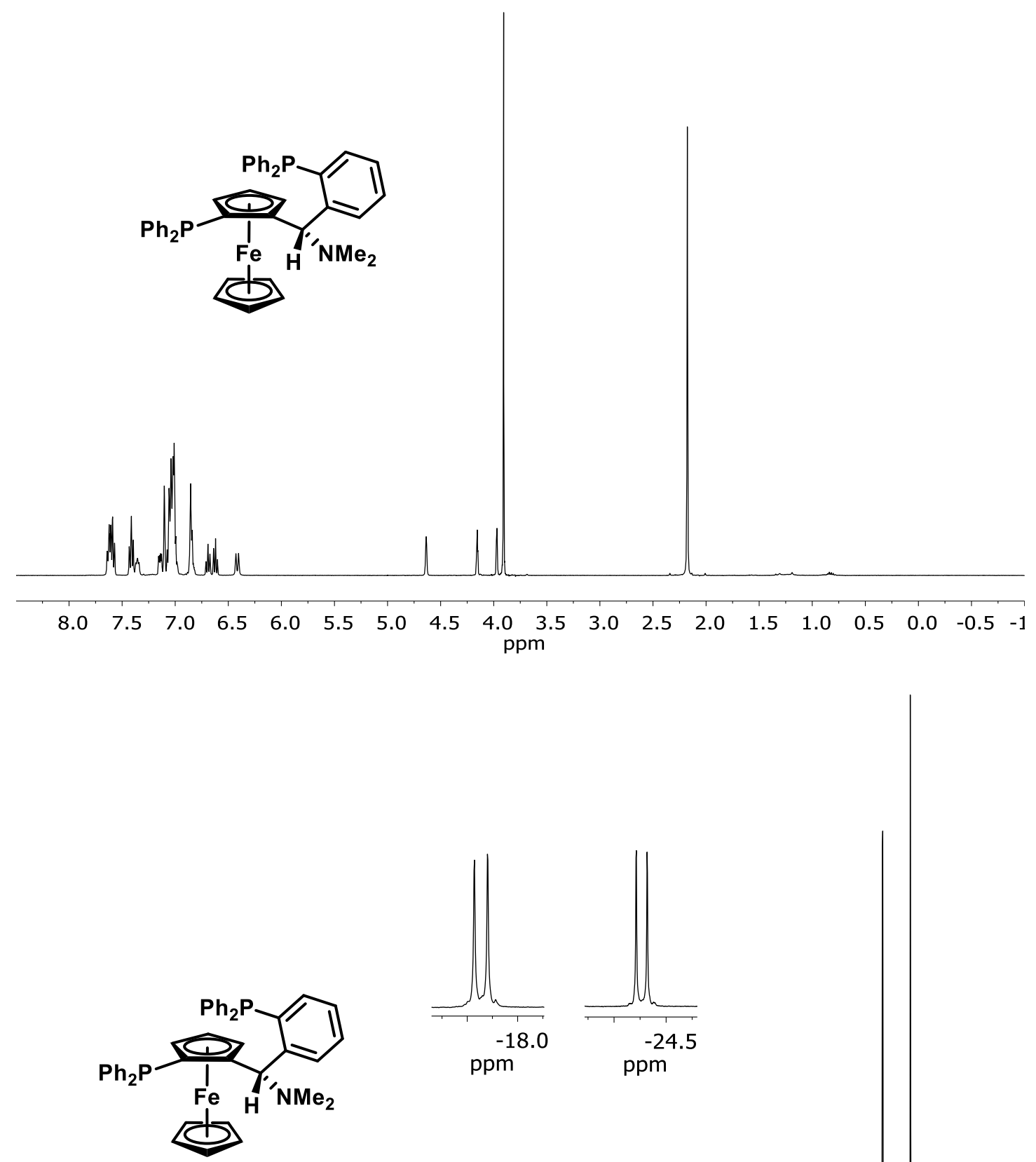

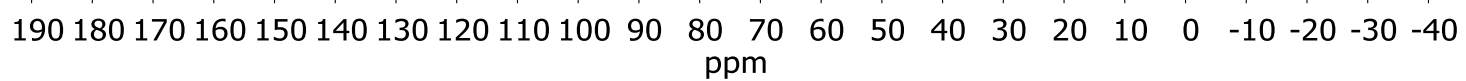


Figure S47. ${ }^{1} \mathrm{H}$ and ${ }^{31} \mathrm{P}$ NMR spectra of ligand 50 in $\mathrm{C}_{6} \mathrm{D}_{6} ;(R, R)-(+)-2,2^{\prime}-\operatorname{bis}[(S)-(N, N$-dimethylamino $)$ (phenyl) methyl]-1,1'-bis(di(2-methylphenyl)phosphino)ferrocene.
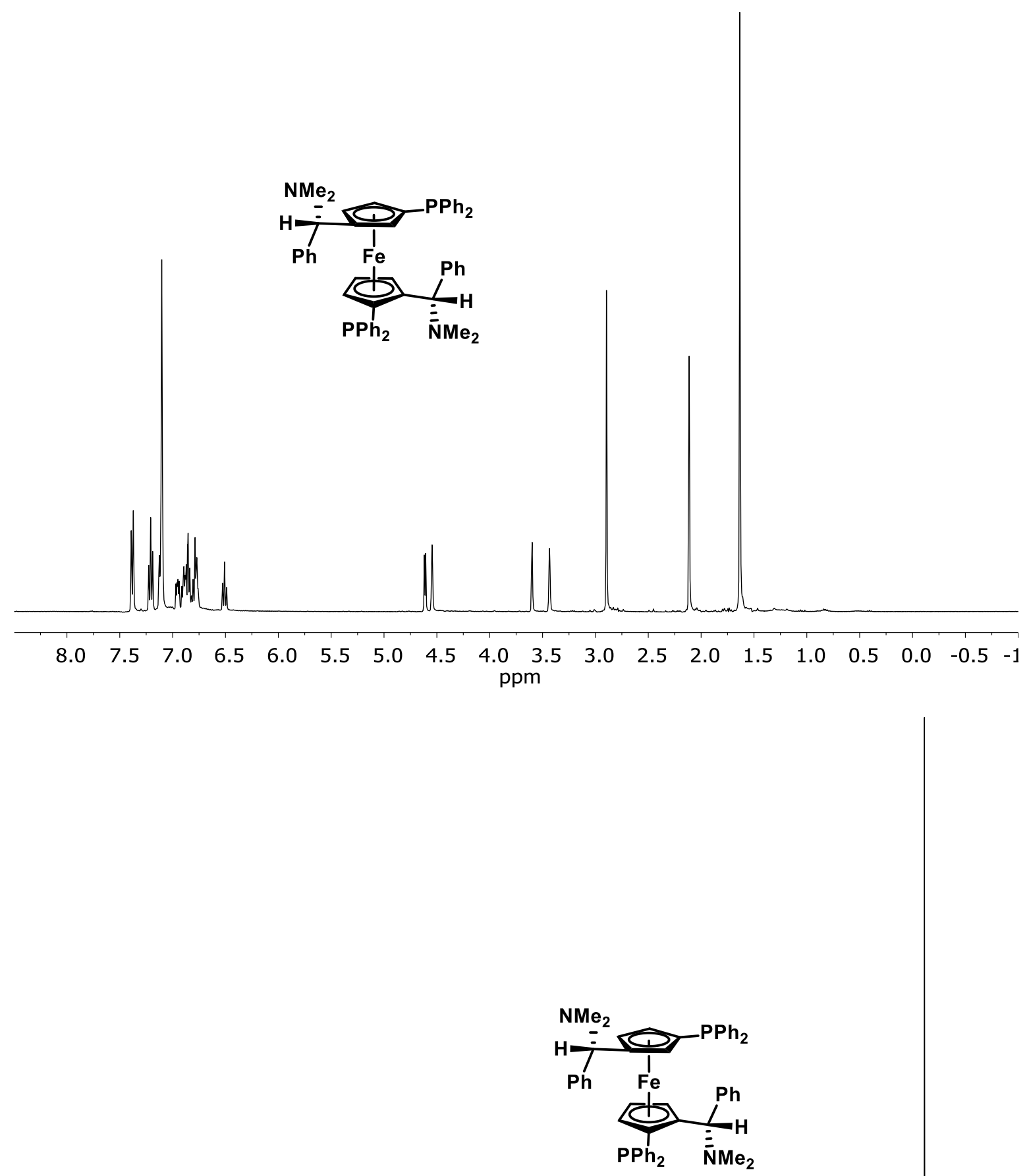

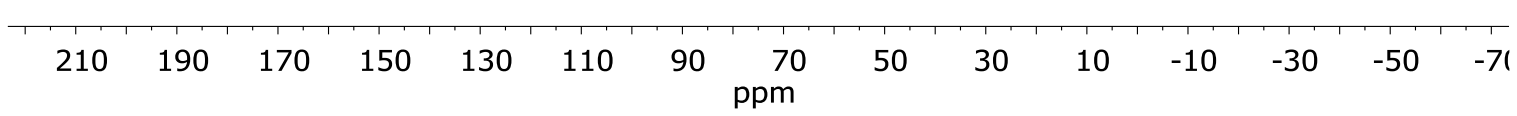


Figure S48. ${ }^{1} \mathrm{H}$ and ${ }^{31} \mathrm{P}$ NMR spectra of ligand 51 in $\mathrm{C}_{6} \mathrm{D}_{6} ; 1,1$ '-[(oxydi-2,1-phenylene)]bis[1,1-diphenyl phosphine].
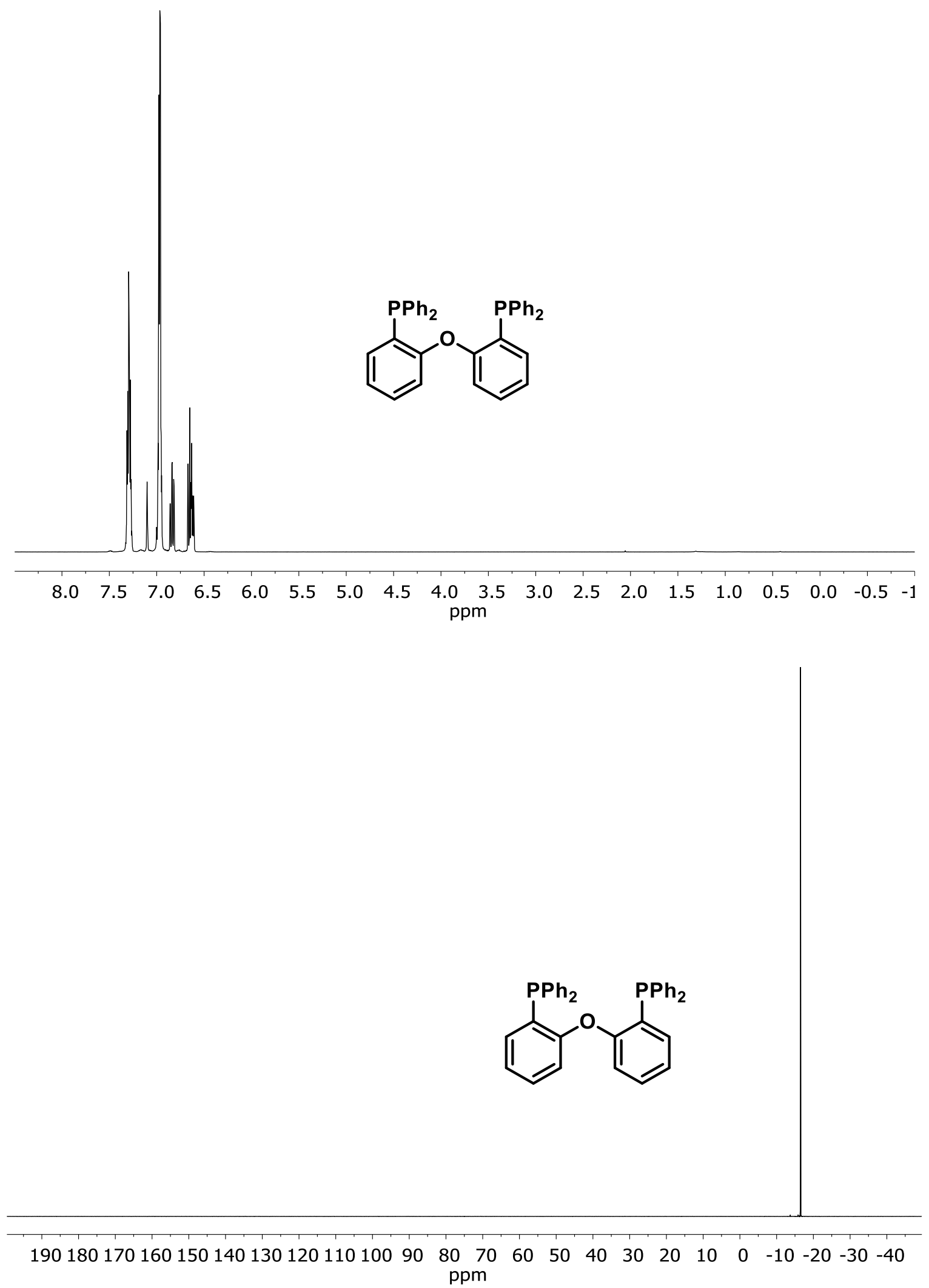
Figure S49. ${ }^{1} \mathrm{H}$ and ${ }^{31} \mathrm{P}$ NMR spectra of ligand $\mathbf{5 2}$ in $\mathrm{CDCl}_{3}$; 4,6-bis(diphenylphosphino)- $10 \mathrm{H}$ phenoxazine.
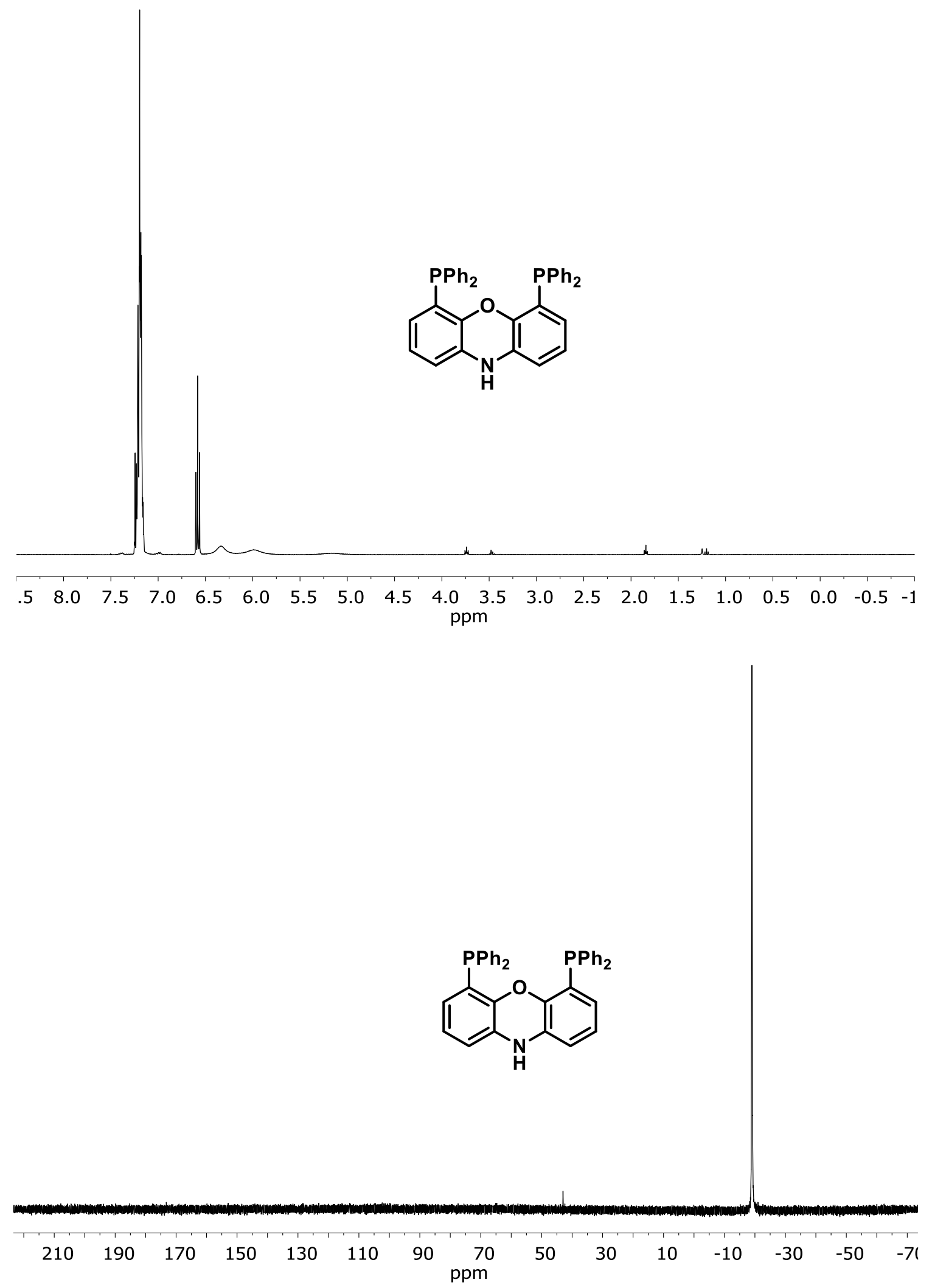
Figure S50. ${ }^{1} \mathrm{H}$ and ${ }^{31} \mathrm{P}$ NMR spectra of ligand 53 in $\mathrm{C}_{6} \mathrm{D}_{6} ; 1,1$ '-[2,7-bis(1,1-dimethylethyl)-9,9-dimethyl9H-xanthene-4,5-diyl]bis[1,1-diphenylphosphine].
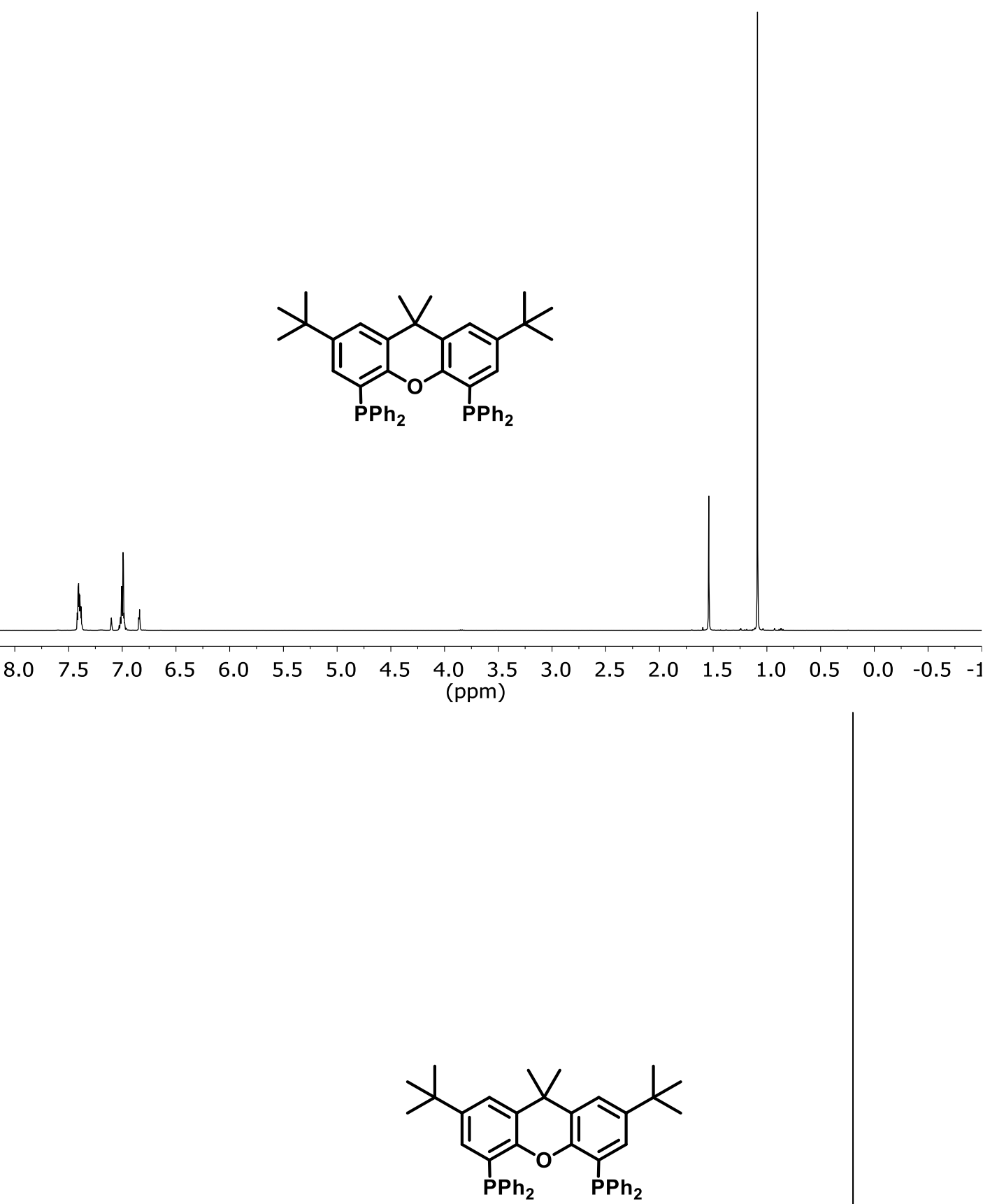

$19018017016015014013012011010090 \quad 80 \quad 70 \quad 60 \begin{array}{llllllllll}40 & 30 & 20 & 10 & 0 & -10 & -20 & -30 & -40\end{array}$ (ppm) 
Figure S51. ${ }^{1} \mathrm{H}$ and ${ }^{31} \mathrm{P}$ NMR spectrum of ligand 54 in $\mathrm{C}_{6} \mathrm{D}_{6} ; 1,1$ '-(oxydi-2,1-phenylene)bis[1,1dicyclohexylphosphine].
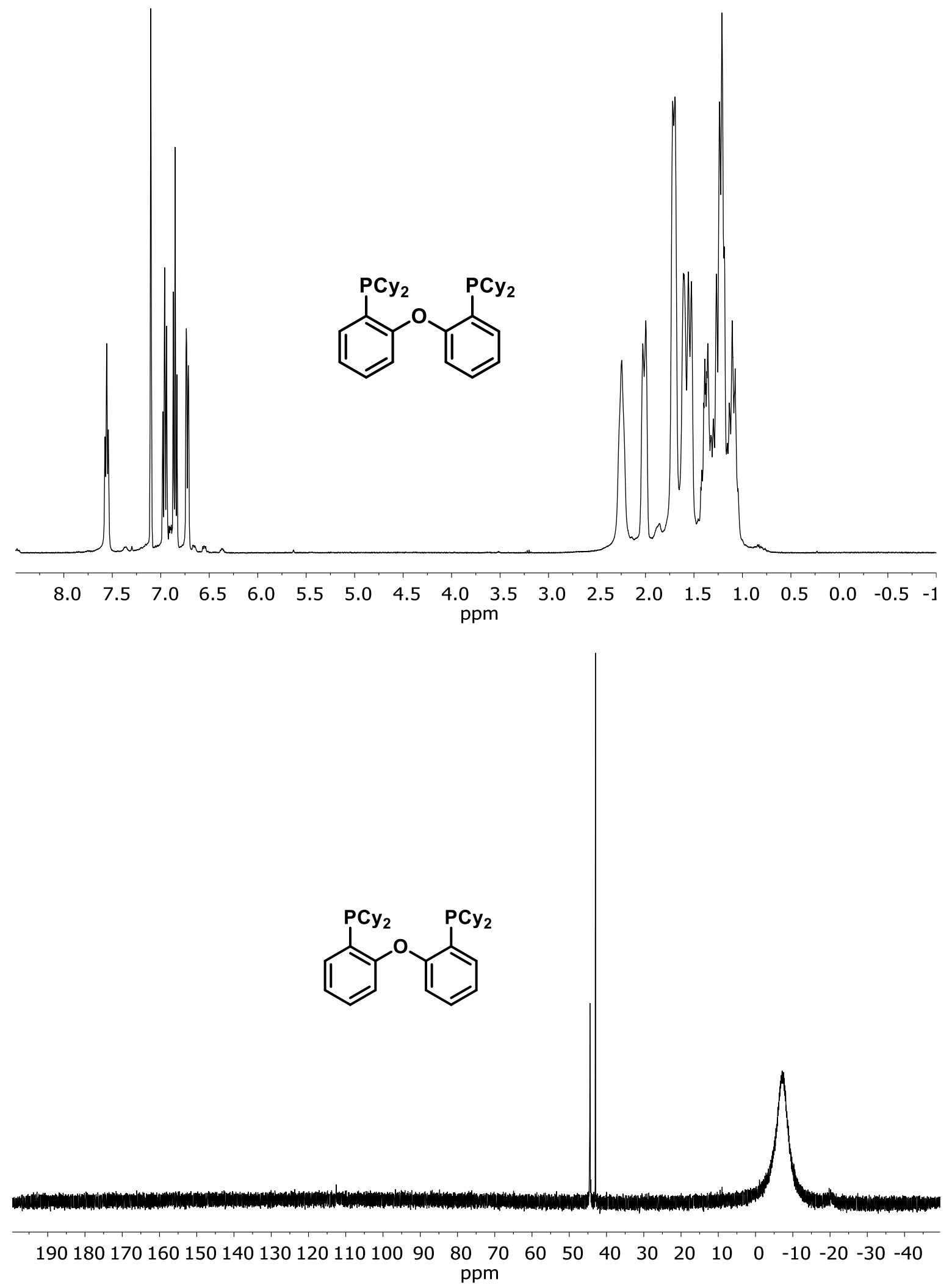
Figure S52. ${ }^{1} \mathrm{H}$ and ${ }^{31} \mathrm{P}$ NMR spectrum of ligand 55 in $\mathrm{C}_{6} \mathrm{D}_{6} ; 1,1$ '-[1,2-phenylenebis(methylene)]bis[1,1bis(1,1-dimethylethyl)phosphine].

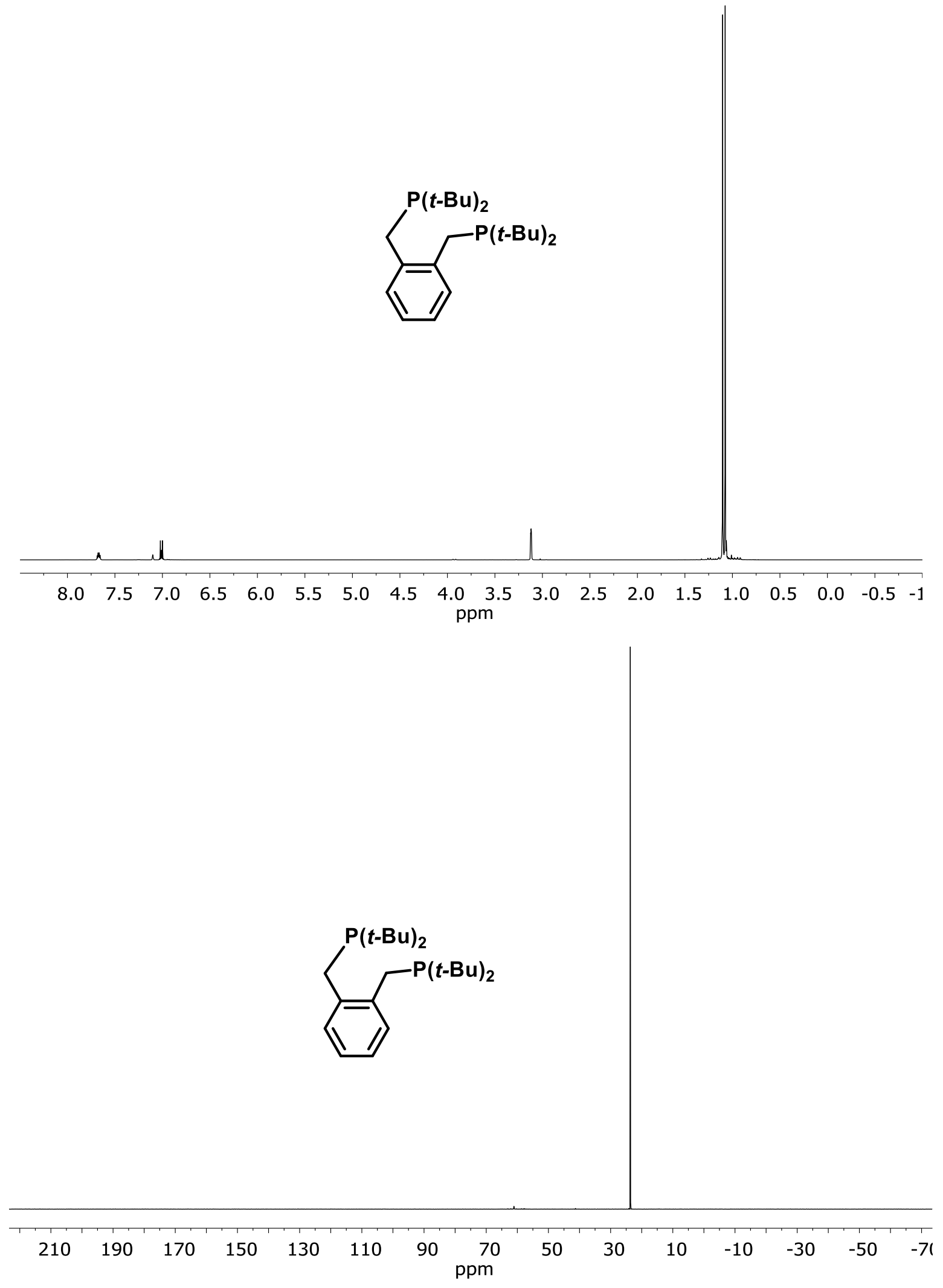


Figure S53. ${ }^{1} \mathrm{H}$ and ${ }^{31} \mathrm{P}$ NMR spectra of ligand 56 in $\mathrm{C}_{6} \mathrm{D}_{6}$; 2,6-bis[[bis(1,1-dimethylethyl)phosphino] methyl]pyridine].

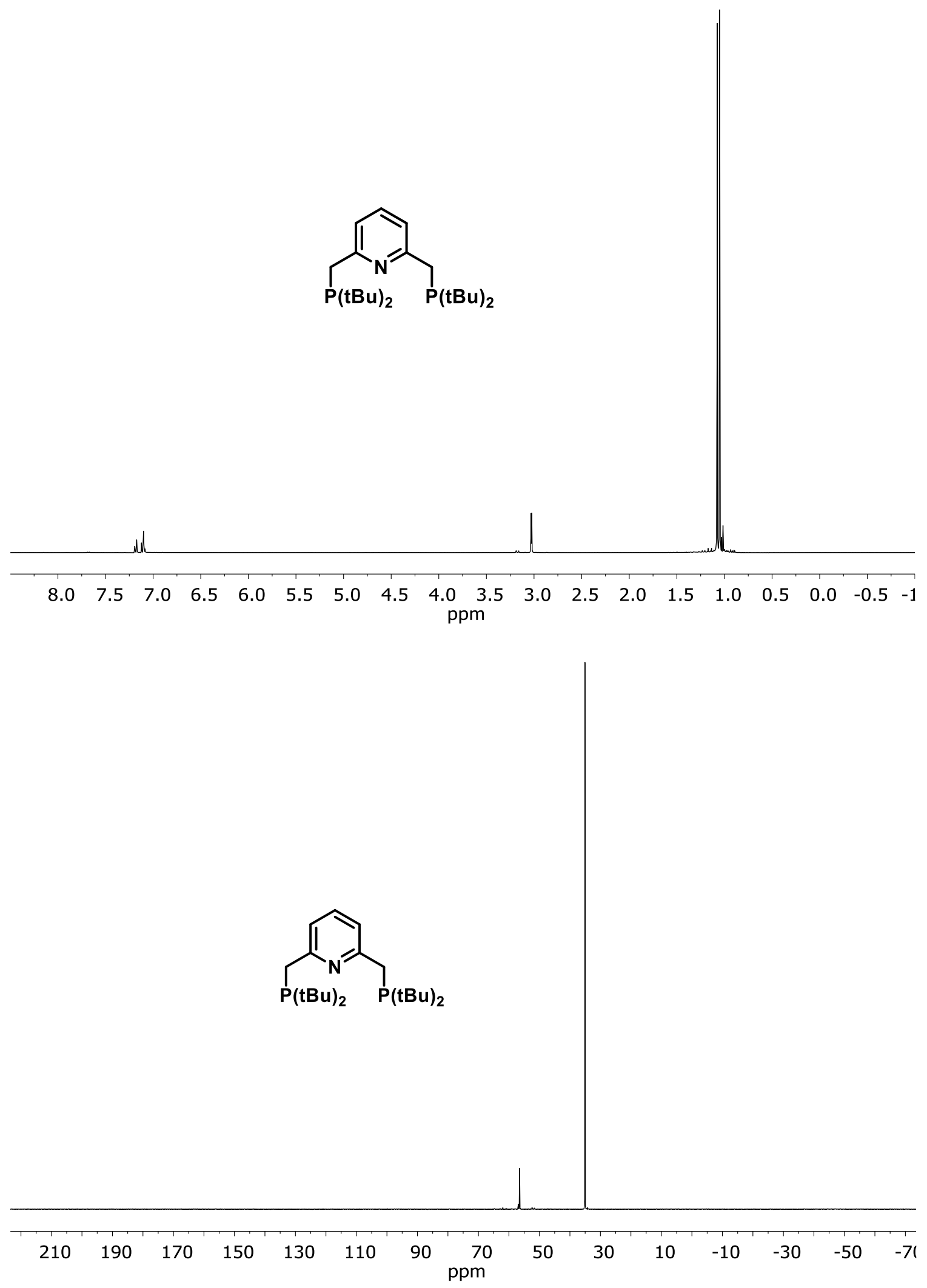


Figure S54. ${ }^{1} \mathrm{H}$ and ${ }^{31} \mathrm{P}$ NMR spectra of ligand 57 in $\mathrm{C}_{6} \mathrm{D}_{6} ; 1,1$ '-(1,2-ethanediyl)bis[1,1-dicyclohexyl phosphine].
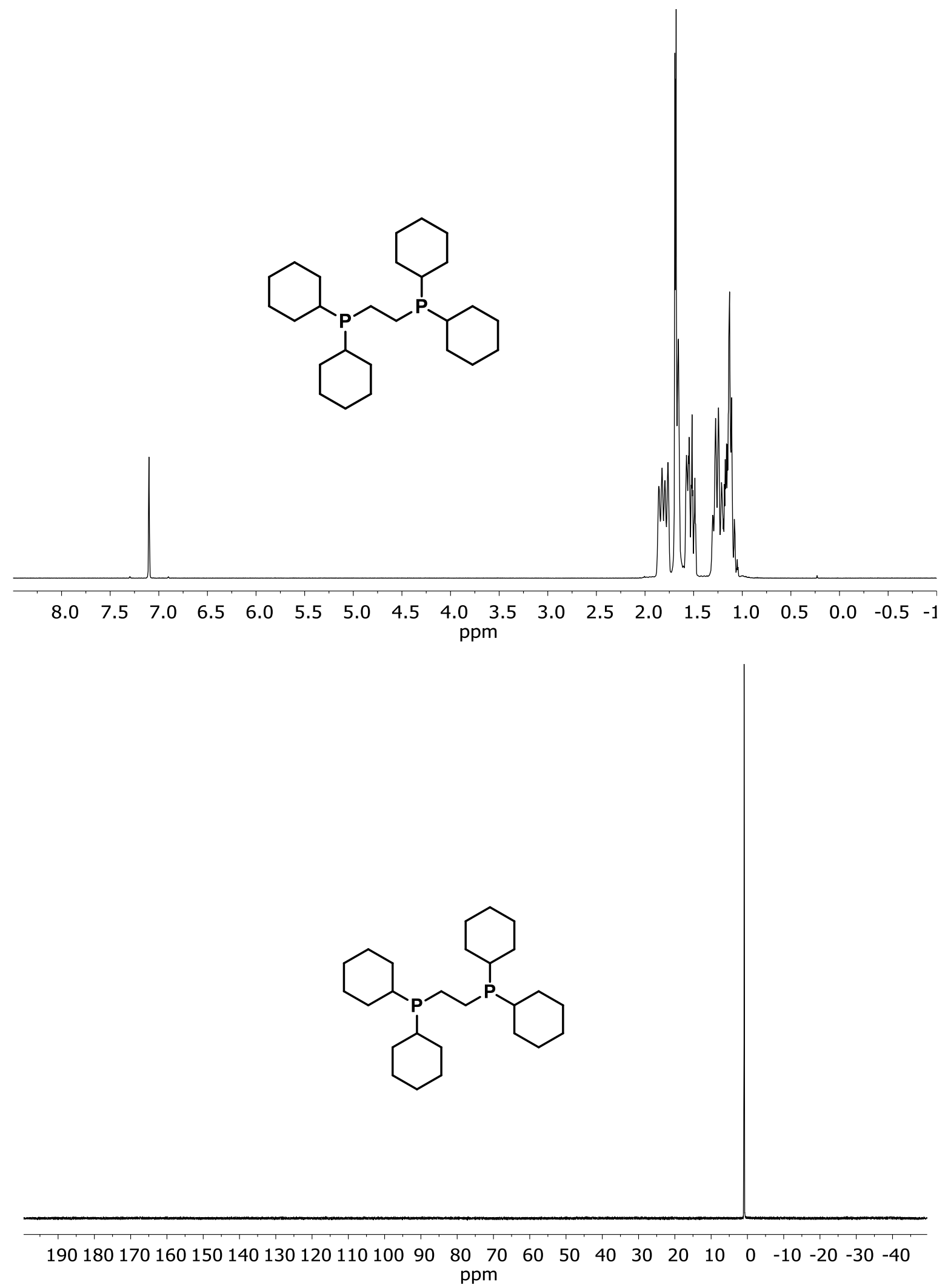


\section{High-Throughput Ethylene Oligomerization Data}

Table S5. Averaged Activities of Cr Catalysts ${ }^{\mathrm{a}}$ Evaluated for Ethylene Oligomerization in the High-Throughput Reactor: Ligands 1 - 32

\begin{tabular}{|c|c|c|c|c|c|c|c|c|c|}
\hline Entry & Compound & $\begin{array}{l}\text { 1-Octene } \\
\text { activity } \\
\text { (g/g Cr hr) }\end{array}$ & $\begin{array}{l}\text { 1-Hexene } \\
\text { activity } \\
\text { (g/g Cr hr) }\end{array}$ & $\begin{array}{c}\text { Methylcyclopentane } \\
\text { activity } \\
\text { (g/g Cr hr) }\end{array}$ & $\begin{array}{c}\text { Methylenecyclopentane } \\
\text { activity } \\
\text { (g/g Cr hr) } \\
\end{array}$ & $\begin{array}{l}\text { Total cyclics } \\
\text { activity } \\
\text { (g/g Cr hr) } \\
\end{array}$ & $\begin{array}{l}\mathrm{C} 10-\mathrm{C} 18^{\mathrm{b}} \\
\text { activity } \\
\text { (g/g Cr hr) }\end{array}$ & $\begin{array}{c}\text { HDPE } \\
\text { activity } \\
\text { (g/g Cr hr) }\end{array}$ & $\begin{array}{c}\text { Total } \\
\text { activity } \\
\text { (g/g Cr hr) }\end{array}$ \\
\hline 1 & 1 & 304,885 & 122,462 & 9,827 & 11,038 & 20,865 & 33,013 & 10,654 & 491,879 \\
\hline 2 & 2 & 85,192 & 37,327 & 4,981 & 8,577 & 13,558 & 8,958 & 13,962 & 158,997 \\
\hline 3 & 3 & 291,942 & 196,712 & 9,231 & 13,038 & 22,269 & 29,355 & 18,692 & 558,970 \\
\hline 4 & 4 & 96,173 & 27,635 & 13,269 & 27,769 & 41,038 & 23,213 & 23,077 & 211,136 \\
\hline 5 & 6 & 252,346 & 101,154 & 20692 & 32,192 & 52,885 & 22683 & 24,885 & 453,952 \\
\hline 6 & 10 & 45,558 & 883,769 & 365 & 500 & 865 & 41,197 & 6154 & 977,543 \\
\hline 7 & 11 & 2,173 & 514,481 & 77 & 96 & 173 & 8,220 & 4,115 & 529,162 \\
\hline 8 & 12 & 4,769 & 31,615 & 327 & 481 & 808 & 1,615 & 21,538 & 60,346 \\
\hline 9 & 13 & 135 & 173 & 0 & 58 & 58 & 138 & 6,115 & 6,618 \\
\hline 10 & 14 & 327 & 18,058 & 0 & 58 & 58 & 2,561 & 90,038 & 111,042 \\
\hline 11 & 15 & 7,923 & 13,538 & 615 & 3,135 & 3,750 & 34,176 & 51,615 & 111,003 \\
\hline 12 & 16 & 1,865 & 346 & 0 & 58 & 58 & 1,139 & 154,038 & 157,447 \\
\hline 13 & 17 & 4,596 & 31,365 & 346 & 654 & 1,000 & 1,785 & 7,615 & 46,362 \\
\hline 14 & 18 & 327 & 750 & 0 & 269 & 269 & 631 & 4,192 & 6,170 \\
\hline 15 & 19 & 77 & 77 & 0 & 96 & 96 & 93 & 5,692 & 6,036 \\
\hline 16 & 20 & 47,788 & 14,673 & 10,288 & 21,596 & 31,885 & 12,164 & 7,962 & 114,471 \\
\hline 17 & 21 & 712 & 14,615 & 77 & 192 & 269 & 825 & 5,269 & 21,691 \\
\hline 18 & 22 & 21,750 & 7,846 & 6,846 & 9,596 & 16,442 & 8,749 & 6,154 & 60,942 \\
\hline 19 & 23 & 118,192 & 167,538 & 11,154 & 21,308 & 32,462 & 25,015 & 5,385 & 367,622 \\
\hline 20 & 24 & 0 & 2,173 & 38 & 115 & 154 & 281 & 5,615 & 8,638 \\
\hline 21 & 25 & 0 & 24,115 & 19 & 77 & 96 & 730 & 5,846 & 31,103 \\
\hline 22 & 26 & 1,038 & 72,385 & 154 & 558 & 712 & 3,242 & 12,346 & 89,723 \\
\hline 23 & 27 & 92,269 & 58,692 & 5,750 & 17,577 & 23,327 & 3,864 & 6,615 & 184,768 \\
\hline 24 & 28 & 17,154 & 3,096 & 1,635 & 3,462 & 5,096 & 9,740 & 10,808 & 45,894 \\
\hline 25 & 29 & 827 & 1,769 & 19 & 77 & 96 & 991 & 26,115 & 29,799 \\
\hline 26 & 30 & 3,538 & 1,827 & 250 & 615 & 865 & 1,822 & 8,269 & 16,322 \\
\hline 27 & 31 & 112,673 & 57,462 & 7,135 & 10,615 & 17,750 & 11,471 & 17,731 & 217,086 \\
\hline 28 & 32 & 274,731 & 180,288 & 8,712 & 12,154 & 20,865 & 26,447 & 11,846 & 514,178 \\
\hline
\end{tabular}

${ }^{a}$ In situ catalyst formation with $\mathrm{CrCl}_{3}(\mathrm{THF})_{3 .} .0 .1 \mu \mathrm{mol} \mathrm{Cr}$, total volume $=5 \mathrm{~mL}$, solvent $=$ methylcyclohexane, $\mathrm{MMAO}-3 \mathrm{~A}: \mathrm{Cr}=1000: 1,60{ }^{\circ} \mathrm{C}$, 500 psi ethylene, 30-minute reaction time, values are averages of duplicates performed in the same library.

${ }^{\mathrm{b}} \mathrm{C} 10$ - C18: Higher $\alpha$-olefins include decenes, dodecenes, tetradecenes, hexadecenes, and octadecenes. GC retention times defined in Table S4 on pg. 10 
Table S6. Averaged Activities of Cr Catalysts ${ }^{\mathrm{a}}$ Evaluated for Ethylene Oligomerization in the High-Throughput Reactor: Ligands 33 - 57

\begin{tabular}{|c|c|c|c|c|c|c|c|c|c|}
\hline Entry & Compound & $\begin{array}{l}\text { 1-Octene } \\
\text { activity } \\
\text { (g/g Cr hr) }\end{array}$ & $\begin{array}{l}\text { 1-Hexene } \\
\text { activity } \\
\text { (g/g Cr hr) }\end{array}$ & $\begin{array}{c}\text { Methylcyclopentane } \\
\text { activity } \\
\text { (g/g Cr hr) }\end{array}$ & $\begin{array}{c}\text { Methylenecyclopentane } \\
\text { activity } \\
\text { (g/g Cr hr) }\end{array}$ & $\begin{array}{l}\text { Total cyclics } \\
\text { activity } \\
\text { (g/g Cr hr) }\end{array}$ & $\begin{array}{l}\mathrm{C} 10-\mathrm{C} 18^{\mathrm{b}} \\
\text { activity } \\
\text { (g/g Cr hr) }\end{array}$ & $\begin{array}{c}\text { HDPE } \\
\text { activity } \\
\text { (g/g Cr hr) }\end{array}$ & $\begin{array}{c}\text { Total } \\
\text { activity } \\
\text { (g/g Cr hr) }\end{array}$ \\
\hline 1 & 33 & 269 & 11,038 & 0 & 77 & 77 & 2,245 & 46,423 & 60,053 \\
\hline 2 & 34 & 500 & 538 & 0 & 77 & 77 & 3,714 & 28,231 & 33,060 \\
\hline 3 & 35 & 173 & 7,173 & 0 & 77 & 77 & 2,708 & 25,231 & 35,362 \\
\hline 4 & 36 & 135 & 2,250 & 0 & 38 & 38 & 956 & 16,077 & 19,456 \\
\hline 5 & 37 & 115 & 269 & 0 & 58 & 58 & 331 & 5,346 & 6,119 \\
\hline 6 & 38 & 212 & 231 & 0 & 77 & 77 & 514 & 8,077 & 9,110 \\
\hline 7 & 39 & 269 & 346 & 0 & 192 & 192 & 1,907 & 9,846 & 12,561 \\
\hline 8 & 40 & 77 & 154 & 0 & 96 & 96 & 190 & 13,346 & 13,863 \\
\hline 9 & 41 & 16,865 & 4,019 & 1,135 & 2,192 & 3,327 & 7,363 & 30,577 & 62,152 \\
\hline 10 & 42 & 192 & 519 & 0 & 58 & 58 & 1,770 & 69,308 & 71,847 \\
\hline 11 & 43 & 38 & 5,558 & 0 & 77 & 77 & 340 & 4,231 & 10,243 \\
\hline 12 & 44 & 404 & 1,058 & 0 & 135 & 135 & 2,282 & 9,154 & 13,032 \\
\hline 13 & 45 & 38 & 115 & 0 & 38 & 38 & 130 & 4,231 & 4,553 \\
\hline 14 & 46 & 0 & 346 & 0 & 231 & 231 & 71 & 5,192 & 5,840 \\
\hline 15 & 47 & 981 & 1,385 & 0 & 250 & 250 & 4,300 & 8,731 & 15,646 \\
\hline 16 & 48 & 3,750 & 3,904 & 0 & 173 & 173 & 17,060 & 16,769 & 41,656 \\
\hline 17 & 49 & 58 & 135 & 0 & 58 & 58 & 137 & 6,808 & 7,194 \\
\hline 18 & 50 & 96 & 135 & 0 & 58 & 58 & 370 & 6,962 & 7,620 \\
\hline 19 & 51 & 712 & 1,846 & 0 & 115 & 115 & 3,475 & 6,577 & 12,725 \\
\hline 20 & 52 & 96 & 500 & 0 & 962 & 962 & 192 & 4,000 & 5,750 \\
\hline 21 & 53 & 250 & 808 & 0 & 558 & 558 & 617 & 5,231 & 7,463 \\
\hline 22 & 54 & 38 & 38 & 0 & 58 & 58 & 45 & 3,654 & 3,833 \\
\hline 23 & 55 & 38 & 96 & 0 & 135 & 135 & 238 & 5,846 & 6,353 \\
\hline 24 & 56 & 0 & 115 & 0 & 0 & 0 & 349 & 4,769 & 5,234 \\
\hline 25 & 57 & 0 & 14,462 & 0 & 77 & 77 & 560 & 6,423 & 21,521 \\
\hline
\end{tabular}

${ }^{a}$ In situ catalyst formation with $\mathrm{CrCl}_{3}(\mathrm{THF})_{3} .0 .1 \mu \mathrm{mol} \mathrm{Cr}$, total volume $=5 \mathrm{~mL}$, solvent $=$ methylcyclohexane, $\mathrm{MMAO}-3 \mathrm{~A}: \mathrm{Cr}=1000: 1,60{ }^{\circ} \mathrm{C}$, 500 psi ethylene, 30-minute reaction time, values are averages of duplicates performed in the same library.

${ }^{\mathrm{b}} \mathrm{C} 10$ - C18: Higher $\alpha$-olefins include decenes, dodecenes, tetradecenes, hexadecenes, and octadecenes. GC retention times defined in Table S4 on pg. 10. 
Table S7. Averaged Selectivities of Cr Catalysts ${ }^{\text {a }}$ Evaluated for Ethylene Oligomerization in the High-Throughput Reactor: Ligands 1 - 32

\begin{tabular}{|c|c|c|c|c|c|c|c|c|c|}
\hline Entry & Ligand \# & $\begin{array}{l}\text { 1-Octene } \\
\text { selectivity } \\
\text { (wt } \%)\end{array}$ & $\begin{array}{l}\text { 1-Hexene } \\
\text { selectivity } \\
\text { (wt } \%)\end{array}$ & $\begin{array}{l}\text { Methylcyclopentane } \\
\text { selectivity } \\
(\mathrm{wt} \%) \\
\end{array}$ & $\begin{array}{c}\text { Methylenecyclopentane } \\
\text { selectivity } \\
\text { (wt } \%)\end{array}$ & $\begin{array}{c}\text { Total cyclics } \\
\text { selectivity } \\
(\mathrm{wt} \%) \\
\end{array}$ & $\begin{array}{l}\mathrm{C} 10-\mathrm{C} 18^{\mathrm{b}} \\
\text { selectivity } \\
\text { (wt } \%)\end{array}$ & $\begin{array}{c}\text { HDPE } \\
\text { selectivity } \\
\text { (wt } \%) \\
\end{array}$ & $\begin{array}{c}\text { Total } \\
\text { selectivity } \\
(\mathrm{wt} \%)\end{array}$ \\
\hline 1 & 1 & 62.0 & 24.9 & 2.0 & 2.2 & 4.2 & 6.7 & 2.2 & 100.0 \\
\hline 2 & 2 & 53.7 & 23.5 & 3.1 & 5.4 & 8.5 & 5.6 & 8.6 & 100.0 \\
\hline 3 & 3 & 52.4 & 35.2 & 1.7 & 2.3 & 4.0 & 5.2 & 3.2 & 100.0 \\
\hline 4 & 4 & 45.4 & 13.0 & 6.2 & 13.0 & 19.2 & 11.1 & 11.0 & 100.0 \\
\hline 5 & 6 & 55.6 & 22.3 & 4.6 & 7.1 & 11.6 & 5.0 & 5.5 & 100.0 \\
\hline 6 & 10 & 4.7 & 90.4 & 0.0 & 0.1 & 0.1 & 4.2 & 0.6 & 100.0 \\
\hline 7 & 11 & 0.4 & 97.2 & 0.0 & 0.0 & 0.0 & 1.6 & 0.8 & 100.0 \\
\hline 8 & 12 & 8.0 & 52.7 & 0.5 & 0.8 & 1.3 & 2.7 & 35.3 & 100.0 \\
\hline 9 & 13 & 2.0 & 2.5 & 0.0 & 0.8 & 0.8 & 2.1 & 92.4 & 100.0 \\
\hline 10 & 14 & 0.3 & 16.3 & 0.0 & 0.1 & 0.1 & 32.3 & 81.1 & 100.0 \\
\hline 11 & 15 & 7.1 & 12.3 & 0.6 & 2.9 & 3.4 & 30.9 & 46.2 & 100.0 \\
\hline 12 & 16 & 1.2 & $\begin{array}{l}0.2 \\
\end{array}$ & 0.0 & 0.0 & 0.0 & $\begin{array}{l}0.7 \\
\end{array}$ & 97.8 & 100.0 \\
\hline 13 & 17 & 9.9 & 67.6 & 0.7 & 1.4 & 2.2 & 3.9 & 16.5 & 100.0 \\
\hline 14 & 18 & 5.1 & 11.8 & 0.0 & 4.2 & 4.2 & 9.9 & 69.0 & 100.0 \\
\hline 15 & 19 & 1.3 & 1.3 & 0.0 & 1.6 & 1.6 & 1.5 & 94.3 & 100.0 \\
\hline 16 & 20 & 41.5 & 12.7 & 9.0 & 18.7 & 27.7 & 10.5 & 7.1 & 100.0 \\
\hline 17 & 21 & 3.2 & 67.2 & 0.3 & 0.9 & 1.2 & 3.8 & 24.6 & 100.0 \\
\hline 18 & 22 & 35.7 & 12.9 & 11.3 & 15.8 & 27.0 & 14.4 & 10.1 & 100.0 \\
\hline 19 & 23 & 33.9 & 48.1 & 3.2 & 6.1 & 9.3 & 7.2 & 1.5 & 100.0 \\
\hline 20 & 24 & 0.0 & 26.8 & 0.5 & 1.4 & 1.9 & 3.4 & 67.9 & 100.0 \\
\hline 21 & 25 & 0.0 & 78.3 & 0.1 & 0.2 & 0.3 & 2.4 & 19.0 & 100.0 \\
\hline 22 & 26 & 1.2 & 79.6 & 0.2 & 0.6 & 0.8 & 3.6 & 14.8 & 100.0 \\
\hline 23 & 27 & 50.0 & 31.8 & 3.1 & 9.5 & 12.6 & 2.1 & 3.5 & 100.0 \\
\hline 24 & 28 & 37.3 & 6.7 & 3.5 & 7.5 & 11.1 & 21.1 & 23.7 & 100.0 \\
\hline 25 & 29 & 2.8 & 5.9 & 0.1 & 0.3 & 0.3 & 3.3 & 87.7 & 100.0 \\
\hline 26 & 30 & 21.6 & 11.1 & 1.5 & 3.7 & 5.2 & 11.0 & $\begin{array}{l}51.1 \\
\end{array}$ & 100.0 \\
\hline 27 & 31 & 52.2 & 26.6 & 3.3 & 4.9 & 8.2 & 5.2 & 7.8 & 100.0 \\
\hline 28 & 32 & 53.4 & 35.0 & 1.7 & 2.4 & 4.1 & 5.1 & 2.3 & 100.0 \\
\hline
\end{tabular}

${ }^{a}$ In situ catalyst formation with $\mathrm{CrCl}_{3}(\mathrm{THF})_{3} .0 .05 \mu$ mol Cr, ligand: $\mathrm{Cr}=1.2: 1$, total volume $=5 \mathrm{~mL}$, solvent $=$ methylcyclohexane, $\mathrm{MMAO}-3 \mathrm{~A}: \mathrm{Cr}$ $=1000: 1,60^{\circ} \mathrm{C}, 500 \mathrm{psi}$ ethylene, 30-minute reaction time, values are averages of duplicates performed in the same library.

${ }^{\mathrm{b}} \mathrm{C} 10$ - C18: Higher $\alpha$-olefins include decenes, dodecenes, tetradecenes, hexadecenes, and octadecenes. GC retention times defined in Table S4 on pg. 10. 
Table S8. Averaged Selectivities of Cr Catalysts ${ }^{\mathrm{a}}$ Evaluated for Ethylene Oligomerization in the High-Throughput Reactor: Ligands 33 - $\mathbf{5 7}$

\begin{tabular}{|c|c|c|c|c|c|c|c|c|c|}
\hline Entry & Ligand \# & $\begin{array}{c}\text { 1-Octene } \\
\text { selectivity } \\
(\mathrm{wt} \%)\end{array}$ & $\begin{array}{c}\text { 1-Hexene } \\
\text { selectivity } \\
\text { (wt } \%)\end{array}$ & $\begin{array}{c}\text { Methylcyclopentane } \\
\text { selectivity } \\
(\mathrm{wt} \%)\end{array}$ & $\begin{array}{c}\text { Methylenecyclopentane } \\
\text { selectivity } \\
(\mathrm{wt} \%)\end{array}$ & $\begin{array}{c}\text { Total cyclics } \\
\text { selectivity } \\
(\mathrm{wt} \%) \\
\end{array}$ & $\begin{array}{l}\text { C10-C18 } \\
\text { selectivity } \\
(\mathrm{wt} \%)\end{array}$ & $\begin{array}{c}\text { HDPE } \\
\text { selectivity } \\
\text { (wt } \%)\end{array}$ & $\begin{array}{c}\text { Total } \\
\text { selectivity } \\
(\mathrm{wt} \%)\end{array}$ \\
\hline 1 & 33 & 0.5 & 18.4 & 0.0 & 0.1 & 0.1 & 3.8 & 77.2 & 100.0 \\
\hline 2 & 34 & 1.5 & 1.7 & 0.0 & 0.3 & 0.3 & 11.0 & 85.5 & 100.0 \\
\hline 3 & 35 & 0.5 & 20.3 & 0.0 & 0.2 & 0.2 & 7.5 & 71.4 & 100.0 \\
\hline 4 & 36 & 0.7 & 11.6 & 0.0 & 0.2 & 0.2 & 5.3 & 82.2 & 100.0 \\
\hline 5 & 37 & 2.3 & 5.4 & 0.0 & 1.3 & 1.3 & 5.8 & 85.1 & 100.0 \\
\hline 6 & 38 & 2.3 & 2.5 & 0.0 & 0.9 & 0.9 & 5.4 & 88.9 & 100.0 \\
\hline 7 & 39 & 2.2 & 2.8 & 0.0 & 1.9 & 1.9 & 15.5 & 77.6 & 100.0 \\
\hline 8 & 40 & 0.5 & 1.1 & 0.0 & 0.7 & 0.7 & 1.5 & 96.2 & 100.0 \\
\hline 9 & 41 & 27.1 & 6.5 & 1.8 & 3.5 & 5.3 & 11.8 & 49.3 & 100.0 \\
\hline 10 & 42 & 0.3 & 0.7 & 0.0 & 0.1 & 0.1 & 2.5 & 96.4 & 100.0 \\
\hline 11 & 43 & 0.4 & 54.4 & 0.0 & 0.8 & 0.8 & 3.1 & 41.3 & 100.0 \\
\hline 12 & 44 & 3.1 & 8.2 & 0.0 & 1.0 & 1.0 & 17.6 & 70.1 & 100.0 \\
\hline 13 & 45 & 0.7 & 2.6 & 0.0 & 0.9 & 0.9 & 3.2 & 92.6 & 100.0 \\
\hline 14 & 46 & 0.0 & 6.3 & 0.0 & 4.0 & 4.0 & 1.3 & 88.5 & 100.0 \\
\hline 15 & 47 & 6.2 & 8.8 & 0.0 & 1.6 & 1.6 & 27.3 & 56.0 & 100.0 \\
\hline 16 & 48 & 9.0 & 9.4 & 0.0 & 0.5 & 0.5 & 40.8 & 40.3 & 100.0 \\
\hline 17 & 49 & 0.7 & 1.9 & 0.0 & 0.8 & 0.8 & 1.8 & 94.8 & 100.0 \\
\hline 18 & 50 & 1.3 & 1.7 & 0.0 & 0.7 & 0.7 & 4.6 & 91.7 & 100.0 \\
\hline 19 & 51 & 5.6 & 14.5 & 0.0 & 0.9 & 0.9 & 27.4 & 51.7 & 100.0 \\
\hline 20 & 52 & 1.4 & 9.2 & 0.0 & 17.7 & 17.7 & 3.2 & 68.5 & 100.0 \\
\hline 21 & 53 & 3.1 & 10.6 & 0.0 & 8.0 & 8.0 & 8.1 & 70.3 & 100.0 \\
\hline 22 & 54 & 0.9 & 1.0 & 0.0 & 1.6 & 1.6 & 1.2 & 95.3 & 100.0 \\
\hline 23 & 55 & 0.5 & 1.6 & 0.0 & 2.7 & 2.7 & 5.4 & 89.9 & 100.0 \\
\hline 24 & 56 & 0.0 & 2.7 & 0.0 & 0.0 & 0.0 & 5.6 & 91.7 & 100.0 \\
\hline 25 & 57 & 0.0 & 67.3 & 0.0 & 0.4 & 0.4 & 2.6 & 29.8 & 100.0 \\
\hline
\end{tabular}

${ }^{a}$ In situ catalyst formation with $\mathrm{CrCl}_{3}(\mathrm{THF})_{3} .0 .05 \mu$ mol Cr, ligand: $\mathrm{Cr}=1.2: 1$, total volume $=5 \mathrm{~mL}$, solvent $=$ methylcyclohexane, $\mathrm{MMAO}-3 \mathrm{~A}: \mathrm{Cr}$ $=1000: 1,60{ }^{\circ} \mathrm{C}, 500 \mathrm{psi}$ ethylene, 30 -minute reaction time, values are averages of duplicates performed in the same library.

${ }^{\mathrm{b}} \mathrm{C} 10$ - C18: Higher $\alpha$-olefins include decenes, dodecenes, tetradecenes, hexadecenes, and octadecenes. GC retention times defined in Table S4 on pg. 10. 
Table S9. GC data for Cr Catalysts Evaluated for Ethylene Oligomerization in the High-Throughput Reactor: Ligands 1 - 24

\begin{tabular}{|c|c|c|c|c|c|c|c|c|c|c|c|}
\hline Entry & Ligand \# & Sample ID & $\begin{array}{c}\text { 1-Octene } \\
\text { (g/g nonane) }\end{array}$ & $\begin{array}{l}\text { 1-Hexene } \\
\text { (g/g nonane) }\end{array}$ & $\begin{array}{c}\text { Methylcyclopentane } \\
\text { (g/g nonane) }\end{array}$ & $\begin{array}{l}\text { Methylenecyclopentane } \\
\text { (g/g nonane) }\end{array}$ & $\begin{array}{c}\text { C10 } \\
\text { (g/g nonane) }\end{array}$ & $\begin{array}{c}\mathrm{C} 12 \\
\text { (g/g nonane) }\end{array}$ & $\begin{array}{c}\mathrm{C} 14 \\
\text { (g/g nonane) }\end{array}$ & $\begin{array}{c}\text { C16 } \\
\text { (g/g nonane) }\end{array}$ & $\begin{array}{c}\mathrm{C} 18 \\
\text { (g/g nonane) }\end{array}$ \\
\hline 1 & 1 & 344114-A1 & 7.482 & 3.033 & 0.2450 & 0.2770 & 0.21473 & 0.29698 & 0.19473 & 0.03925 & 0.03458 \\
\hline 2 & 1 & 344114-D6 & 8.372 & 3.335 & 0.266 & 0.297 & 0.25597 & 0.36111 & 0.2241 & 0.04409 & 0.05116 \\
\hline 3 & 2 & 344108-A5 & 2.073 & 0.913 & 0.123 & 0.208 & 0.0504 & 0.03307 & 0.04267 & 0.02758 & 0.03104 \\
\hline 4 & 2 & 344108-D2 & 2.357 & 1.028 & 0.136 & 0.238 & 0.06619 & 0.04973 & 0.0625 & 0.0434 & 0.05925 \\
\hline 5 & 3 & 344108-A4 & 6.524 & 4.273 & 0.206 & 0.286 & 0.17227 & 0.23469 & 0.1029 & 0.0206 & 0.02834 \\
\hline 6 & 3 & 344108-D3 & 8.657 & 5.956 & 0.274 & 0.392 & 0.27847 & 0.39743 & 0.19533 & 0.04495 & 0.05147 \\
\hline 7 & 4 & 344108-B1 & 2.73 & 0.826 & 0.389 & 0.843 & 0.18387 & 0.08076 & 0.145 & 0.08906 & 0.09583 \\
\hline 8 & 4 & 344108-C6 & 2.271 & 0.611 & 0.301 & 0.601 & 0.17418 & 0.08317 & 0.14971 & 0.09931 & 0.10619 \\
\hline 9 & 6 & $344543-\mathrm{A} 2$ & 6.683 & 2.659 & 0.548 & 0.85 & 0.1932 & 0.14356 & 0.1517 & 0.0623 & 0.05880 \\
\hline 10 & 6 & 344543-D5 & 6.439 & 2.601 & 0.528 & 0.824 & 0.1932 & 0.13065 & 0.1392 & 0.0587 & 0.04814 \\
\hline 12 & 10 & 344543-D4 & 1.096 & 21.204 & 0.009 & 0.012 & 0.85283 & 0.05917 & 0.03046 & 0.002967367 & 0.00324 \\
\hline 13 & 11 & $344122-\mathrm{A} 5$ & 0.056 & 13.291 & 0.002 & 0.003 & 0.20859 & 0.0 & 0.00159 & 0.0 & 0.0 \\
\hline 14 & 11 & 344122-D2 & 0.057 & 13.462 & 0.002 & 0.002 & 0.21547 & 0.00103 & 0.00075 & 0.0 & 0.0 \\
\hline 15 & 12 & 344108-B5 & 0.128 & 0.849 & 0.009 & 0.013 & 0.01136 & 0.00518 & 0.00912 & 0.00648 & 0.01148 \\
\hline 16 & 12 & $344108-\mathrm{C} 2$ & 0.12 & 0.795 & 0.008 & 0.012 & 0.01032 & 0.00585 & 0.00811 & 0.00657 & 0.0095 \\
\hline 17 & 13 & 344126-A4 & 0.003 & 0.003 & 0.0 & 0.001 & 0.00178 & 0.00035 & 0.00066 & 0.00058 & 0.00059 \\
\hline 18 & 13 & 344126-D3 & 0.004 & 0.006 & 0.0 & 0.002 & 0.00174 & 0.00029 & 0.00054 & 0.00062 & 0.0 \\
\hline 19 & 14 & 344114-B2 & 0.009 & 0.497 & 0.0 & 0.001 & 0.01129 & 0.01134 & 0.01628 & 0.01433 & 0.01639 \\
\hline 20 & 14 & 344114-C5 & 0.008 & 0.442 & 0.0 & 0.002 & 0.00962 & 0.00971 & 0.01482 & 0.01198 & 0.01742 \\
\hline 21 & 15 & 344114-B5 & 0.186 & 0.342 & 0.015 & 0.091 & 0.18038 & 0.171 & 0.19204 & 0.14896 & 0.14661 \\
\hline 22 & 15 & 344114-C2 & 0.226 & 0.362 & 0.017 & 0.072 & 0.21316 & 0.2 & 0.19577 & 0.17264 & 0.15658 \\
\hline 24 & 16 & 344126-C5 & 0.053 & 0.01 & 0.0 & 0.002 & 0.00844 & 0.00845 & 0.00543 & 0.00309 & 0.01034 \\
\hline 25 & 17 & 344118-B2 & 0.115 & 0.757 & 0.008 & 0.016 & 0.00865 & 0.00578 & 0.00982 & 0.00786 & 0.02507 \\
\hline 26 & 17 & 344118-C5 & 0.124 & 0.874 & 0.01 & 0.018 & 0.00903 & 0.00567 & 0.00865 & 0.00677 & 0.00554 \\
\hline 27 & 18 & 344122-B4 & 0.005 & 0.011 & 0.0 & 0.003 & $\begin{array}{l}0.005 \\
\end{array}$ & 0.00167 & 0.00093 & 0.00055 & 0.00057 \\
\hline 28 & 18 & $344122-\mathrm{C} 3$ & 0.012 & 0.028 & 0.0 & 0.011 & 0.01129 & 0.00593 & 0.00372 & 0.0023 & 0.00087 \\
\hline 29 & 19 & $344122-\mathrm{A} 2$ & 0.0 & 0.002 & 0.0 & 0.002 & 0.00187 & 0.0 & 0.0 & 0.0 & 0.0 \\
\hline 30 & 19 & 344122-D5 & 0.004 & 0.002 & 0.0 & 0.003 & 0.00225 & 0.00041 & 0.00032 & 0.0 & 0.0 \\
\hline 31 & 20 & 344122-B1 & 1.037 & 0.317 & 0.232 & 0.459 & 0.1056 & 0.03922 & 0.06312 & 0.02785 & 0.02343 \\
\hline 32 & 20 & 344122-C6 & 1.448 & 0.446 & 0.303 & 0.664 & 0.14973 & 0.05603 & 0.08982 & 0.039 & 0.03871 \\
\hline 33 & 21 & 344445-A5 & 0.011 & 0.339 & 0.001 & 0.004 & 0.00998 & 0.00175 & 0.00408 & 0.00133 & 0.00128 \\
\hline 34 & 21 & 344445-D2 & 0.026 & 0.421 & 0.003 & 0.006 & 0.0123 & 0.00224 & 0.00489 & 0.00232 & 0.00274 \\
\hline 35 & 22 & $344543-\mathrm{B} 2$ & 0.508 & 0.185 & 0.164 & 0.226 & 0.08764 & 0.03074 & 0.04679 & 0.02253 & 0.01696 \\
\hline 36 & 22 & 344543-C5 & 0.623 & 0.223 & 0.192 & 0.273 & 0.10700 & 0.0375 & 0.05762 & 0.02762 & 0.02051 \\
\hline 37 & 23 & 344543-B3 & 3.247 & 4.581 & 0.307 & 0.584 & 0.38814 & 0.23944 & 0.05544 & 0.00411 & 0.00205 \\
\hline 38 & 23 & 344543-C4 & 2.899 & 4.131 & 0.273 & 0.524 & 0.34629 & 0.21241 & 0.04999 & 0.00227 & 0.00062 \\
\hline 39 & 24 & 344543-B1 & 0.0 & 0.042 & 0.001 & 0.003 & 0.00284 & 0.00028 & 0.00023 & 0.00012 & 0.00512 \\
\hline 40 & 24 & $344543-\mathrm{C} 6$ & 0.0 & 0.071 & 0.001 & 0.003 & 0.00372 & 0.00042 & 0.00038 & 0.00051 & 0.00096 \\
\hline
\end{tabular}


Table S10. GC data for Cr Catalysts Evaluated for Ethylene Oligomerization in the High-Throughput Reactor: Ligand 25 - 44

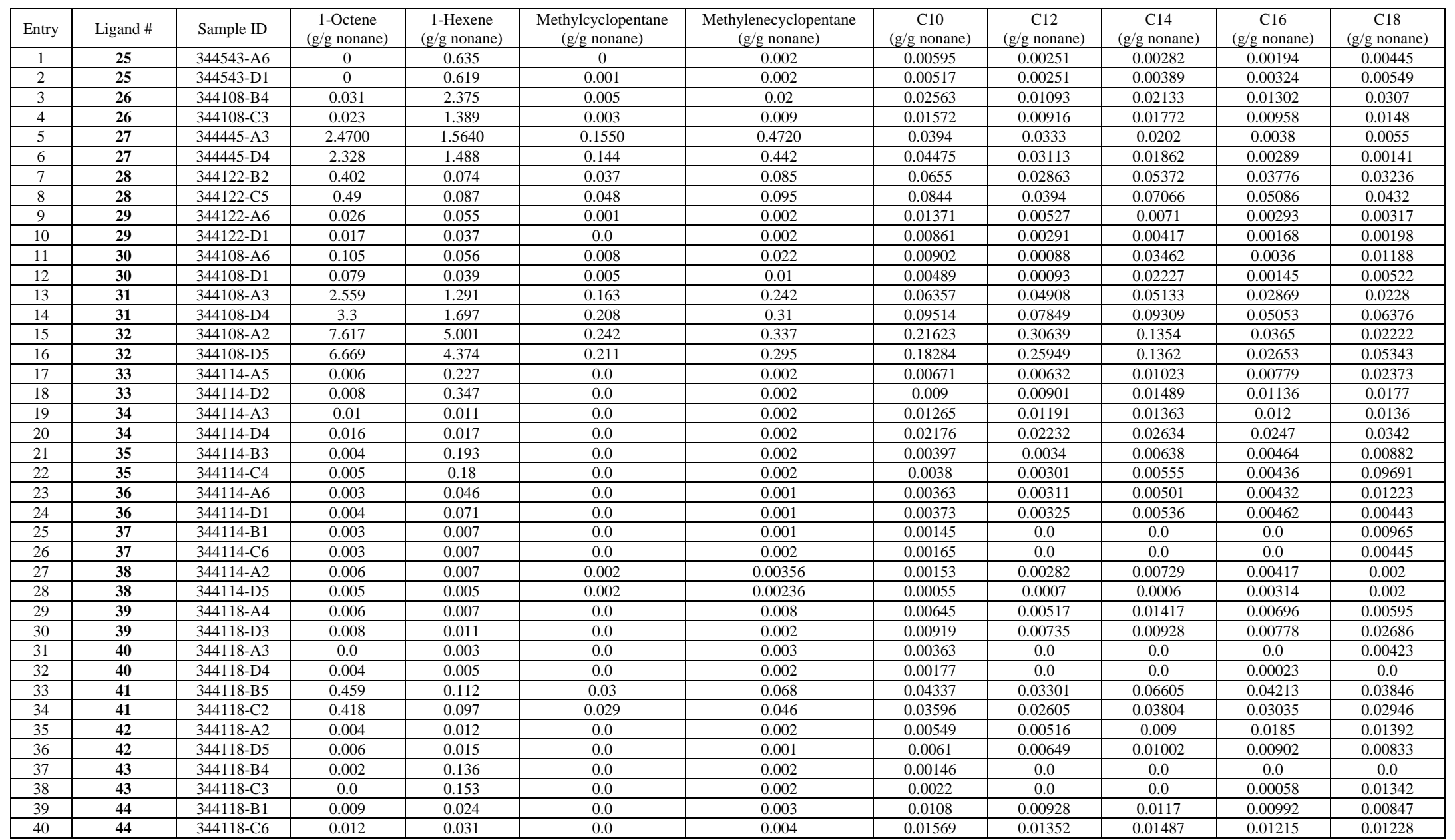


Table S11. GC data for Cr Catalysts Evaluated for Ethylene Oligomerization in the High-Throughput Reactor: Ligands 45 - 57

\begin{tabular}{|c|c|c|c|c|c|c|c|c|c|c|c|}
\hline Entry & Ligand \# & $\begin{array}{l}\text { HOPR } \\
\text { library }\end{array}$ & $\begin{array}{c}\text { 1-Octene } \\
\text { (g/g nonane) }\end{array}$ & $\begin{array}{l}\text { 1-Hexene } \\
\text { (g/g nonane) }\end{array}$ & $\begin{array}{l}\text { Methylcyclopentane } \\
\text { (g/g nonane) }\end{array}$ & $\begin{array}{l}\text { Methylenecyclopentane } \\
\text { (g/g nonane) }\end{array}$ & $\begin{array}{c}\text { C10 } \\
\text { (g/g nonane) }\end{array}$ & $\begin{array}{c}\mathrm{C} 12 \\
\text { (g/g nonane) }\end{array}$ & $\begin{array}{c}\mathrm{C} 14 \\
(\mathrm{~g} / \mathrm{g} \text { nonane })\end{array}$ & $\begin{array}{c}\text { C16 } \\
\text { (g/g nonane) }\end{array}$ & $\begin{array}{c}\text { C18 } \\
\text { (g/g nonane) }\end{array}$ \\
\hline 1 & 45 & 344126-A2 & 0.0 & 0.003 & $\begin{array}{l}0.0 \\
\end{array}$ & 0.001 & 0.00115 & 0.0 & 0.0 & 0.0 & 0.00448 \\
\hline 2 & 45 & 344126-D5 & 0.002 & 0.003 & 0.0 & 0.001 & 0.00114 & 0.0 & 0.0 & 0.0 & 0.0 \\
\hline 3 & 46 & 344126-A3 & 0.0 & 0.007 & 0.0 & 0.004 & 0.0013 & 0.0 & 0.0 & 0.0 & 0.0 \\
\hline 4 & 46 & 344126-D4 & 0.0 & 0.011 & 0.0 & 0.008 & 0.0024 & 0.0 & 0.0 & 0.0 & 0.0 \\
\hline 5 & 47 & 344126-A6 & 0.022 & 0.033 & 0.0 & 0.006 & 0.0227 & 0.0196 & 0.02055 & 0.01565 & 0.01358 \\
\hline 6 & 47 & 344126-D1 & 0.029 & 0.039 & 0.0 & 0.007 & 0.03173 & 0.02818 & 0.02865 & 0.02301 & 0.01994 \\
\hline 7 & 48 & 344118-A6 & 0.064 & 0.067 & 0.0 & 0.004 & 0.07503 & 0.06346 & 0.0617 & 0.04857 & 0.04096 \\
\hline 8 & 48 & 344118-D1 & 0.131 & 0.136 & 0.0 & 0.005 & 0.15403 & 0.13589 & 0.12435 & 0.10021 & 0.08293 \\
\hline 9 & 49 & 344118-A5 & 0.0 & 0.003 & 0.0 & 0.002 & 0.00114 & 0.0 & 0.0 & 0.0 & 0.0 \\
\hline 10 & 49 & 344118-D2 & 0.003 & 0.004 & 0.0 & 0.001 & 0.00113 & 0.0 & 0.0 & 0.00263 & 0.00221 \\
\hline 12 & 50 & 344118-C4 & 0.003 & 0.005 & 0.0 & 0.002 & 0.0015 & 0.0 & 0.0 & 0.0 & 0.01641 \\
\hline 13 & 51 & 344122-B3 & 0.017 & 0.044 & 0.0 & 0.003 & 0.02144 & 0.01881 & 0.01932 & 0.01518 & 0.01394 \\
\hline 14 & 51 & 344122-C4 & 0.02 & 0.052 & 0.0 & 0.003 & 0.02332 & 0.01935 & 0.01988 & 0.01514 & 0.01434 \\
\hline 15 & 52 & $344122-B 5$ & 0.0 & 0.014 & 0.0 & 0.027 & 0.00294 & 0.0 & 0.0 & 0.0 & 0.0 \\
\hline 16 & 52 & $344122-\mathrm{C} 2$ & 0.005 & 0.012 & 0.0 & 0.023 & 0.0046 & 0.00141 & 0.00103 & 0.0 & 0.0 \\
\hline 17 & 53 & 344122-A4 & 0.0 & 0.013 & 0.0 & 0.025 & 0.00698 & 0.00201 & 0.00171 & 0.0 & 0.0 \\
\hline 18 & 53 & 344122-D3 & 0.013 & 0.029 & 0.0 & 0.004 & 0.01123 & 0.00526 & 0.00277 & 0.00155 & 0.00059 \\
\hline 19 & 54 & 344122-A3 & 0.002 & 0.001 & 0.0 & 0.001 & 0.00116 & 0.0 & 0.0 & 0.0 & 0.0 \\
\hline 20 & 54 & 344122-D4 & 0.0 & 0.001 & 0.0 & 0.002 & 0.00116 & 0.0 & 0.0 & 0.0 & 0.0 \\
\hline 21 & 55 & 344126-B1 & 0.0 & 0.002 & 0.0 & 0.005 & 0.00266 & 0.0 & 0.00857 & 0.0 & 0.0 \\
\hline 22 & 55 & 344126-C6 & 0.002 & 0.003 & 0.0 & 0.002 & 0.00114 & 0.0 & 0.0 & 0.0 & 0.0 \\
\hline 24 & 56 & 344126-D2 & 0.0 & 0.002 & 0.0 & 0.0 & 0.00219 & 0.0 & 0.01406 & 0.0 & 0.0 \\
\hline 25 & 57 & 344445-A6 & 0.0 & 0.395 & 0.0 & 0.002 & 0.00937 & 0.00045 & 0.00114 & 0.00031 & 0.00112 \\
\hline 26 & 57 & 344445-D1 & 0.0 & 0.357 & 0.0 & 0.002 & 0.00946 & 0.00088 & 0.00305 & 0.001 & 0.00233 \\
\hline
\end{tabular}


Table S12. Products Produced (mg) for Cr Catalysts Evaluated for Ethylene Oligomerization in the High-Throughput Reactor: Ligands 1

$-24$

\begin{tabular}{|c|c|c|c|c|c|c|c|c|c|c|c|c|c|}
\hline Entry & Ligand \# & Sample ID & $\begin{array}{c}\text { 1-Octene } \\
(\mathrm{mg})\end{array}$ & $\begin{array}{c}\text { 1-Hexene } \\
(\mathrm{mg})\end{array}$ & $\begin{array}{l}\text { Methylcyclopentane } \\
\text { (mg) }\end{array}$ & $\begin{array}{l}\text { Methylenecyclopentane } \\
\text { (mg) }\end{array}$ & $\begin{array}{l}\mathrm{C} 10 \\
(\mathrm{mg})\end{array}$ & $\begin{array}{l}\mathrm{C} 12 \\
(\mathrm{mg})\end{array}$ & $\begin{array}{l}\text { C14 } \\
(\mathrm{mg})\end{array}$ & $\begin{array}{l}\text { C16 } \\
(\mathrm{mg})\end{array}$ & $\begin{array}{l}\mathrm{C} 18 \\
(\mathrm{mg})\end{array}$ & $\begin{array}{c}\mathrm{HDPE} \\
(\mathrm{mg})\end{array}$ & $\begin{array}{l}\text { Total } \\
(\mathrm{mg})\end{array}$ \\
\hline 1 & 1 & 344114-A1 & 374.1 & 151.7 & 12.3 & 13.9 & 10.7 & 14.8 & 9.7 & 2.0 & 1.7 & 15.2 & 606.1 \\
\hline 2 & 1 & 344114-D6 & 418.6 & 166.8 & 13.3 & 14.9 & 12.8 & 18.1 & 11.2 & 2.2 & 2.6 & 12.5 & 672.8 \\
\hline 3 & 2 & 344108-A5 & 103.7 & 45.7 & 6.2 & 10.4 & 2.5 & 1.7 & 2.1 & 1.4 & 1.6 & 13.4 & 188.5 \\
\hline 4 & 2 & $344108-\mathrm{D} 2$ & 117.9 & 51.4 & 6.8 & 11.9 & 3.3 & 2.5 & 3.1 & 2.2 & 3.0 & 22.9 & 224.9 \\
\hline 5 & 3 & 344108-A4 & 326.2 & 213.7 & 10.3 & 14.3 & 8.6 & 11.7 & 5.1 & 1.0 & 1.4 & 14.6 & 607.0 \\
\hline 6 & 3 & 344108-D3 & 432.9 & 297.8 & 13.7 & 19.6 & 13.9 & 19.9 & 9.8 & 2.2 & 2.6 & 34.0 & 846.3 \\
\hline 7 & 4 & 344108-B1 & 136.5 & 41.3 & 19.5 & 42.2 & 9.2 & 4.0 & 7.3 & 4.5 & 4.8 & 30.5 & 299.6 \\
\hline 8 & 4 & $344108-\mathrm{C} 6$ & 113.6 & 30.6 & 15.1 & 30.1 & 8.7 & 4.2 & 7.5 & 5.0 & 5.3 & 29.5 & 249.3 \\
\hline 9 & 6 & $344543-\mathrm{A} 2$ & 334.2 & 133.0 & 27.4 & 42.5 & 9.7 & 7.2 & 7.6 & 3.1 & 2.9 & 32.9 & 600.4 \\
\hline 10 & 6 & 344543-D5 & 322.0 & 130.1 & 26.4 & 41.2 & 9.7 & 6.5 & 7.0 & 2.9 & 2.4 & 31.8 & 579.9 \\
\hline 11 & 10 & 344543-A3 & 63.7 & 1237.6 & 0.5 & 0.7 & 51.9 & 3.6 & 3.2 & 0.2 & 0.8 & 8.2 & 1370.3 \\
\hline 12 & 10 & 344543-D4 & 54.8 & 1060.2 & 0.5 & 0.6 & 42.6 & 3.0 & 1.5 & 0.1 & 0.2 & 7.8 & 1171.3 \\
\hline 13 & 11 & 344122-A5 & 2.8 & 664.6 & 0.1 & 0.2 & 10.4 & 0.0 & 0.1 & 0.0 & 0.0 & 5.1 & 683.2 \\
\hline 14 & 11 & 344122-D2 & 2.9 & 673.1 & 0.1 & 0.1 & 10.8 & 0.1 & 0.0 & 0.0 & 0.0 & 5.6 & 692.6 \\
\hline 15 & 12 & 344108-B5 & 6.4 & 42.5 & 0.5 & 0.7 & 0.6 & 0.3 & 0.5 & 0.3 & 0.6 & 21.2 & 73.3 \\
\hline 16 & 12 & $344108-\mathrm{C} 2$ & 6.0 & 39.8 & 0.4 & 0.6 & 0.5 & 0.3 & 0.4 & 0.3 & 0.5 & 34.8 & 83.6 \\
\hline 17 & 13 & $344126-\mathrm{A} 4$ & 0.2 & 0.2 & 0.0 & 0.1 & 0.1 & 0.0 & 0.0 & 0.0 & 0.0 & 7.0 & 7.5 \\
\hline 18 & 13 & 344126-D3 & 0.2 & 0.3 & 0.0 & 0.1 & 0.1 & 0.0 & 0.0 & 0.0 & 0.0 & 8.9 & 9.7 \\
\hline 19 & 14 & 344114-B2 & 0.5 & 24.9 & 0.0 & 0.1 & 0.6 & 0.6 & 0.8 & 0.7 & 0.8 & 122.3 & 151.1 \\
\hline 20 & 14 & 344114-C5 & 0.4 & 22.1 & 0.0 & 0.1 & 0.5 & 0.5 & 0.7 & 0.6 & 0.9 & 111.8 & 137.6 \\
\hline 21 & 15 & 344114-B5 & 9.3 & 17.1 & 0.8 & 4.6 & 9.0 & 8.6 & 9.6 & 7.4 & 7.3 & 56.8 & 130.4 \\
\hline 22 & 15 & $344114-\mathrm{C} 2$ & 11.3 & 18.1 & 0.9 & 3.6 & 10.7 & 10.0 & 9.8 & 8.6 & 7.8 & 77.4 & 158.2 \\
\hline 23 & 16 & $344126-\mathrm{B} 2$ & 2.2 & 0.4 & 0.0 & 0.1 & 0.4 & 0.4 & 0.2 & 0.1 & 0.1 & 201.0 & 204.8 \\
\hline 24 & 16 & $344126-\mathrm{C} 5$ & 2.7 & 0.5 & 0.0 & 0.1 & 0.4 & 0.4 & 0.3 & 0.2 & 0.5 & 199.5 & 204.5 \\
\hline 25 & 17 & 344118-B2 & 5.8 & 37.9 & 0.4 & 0.8 & 0.4 & 0.3 & 0.5 & 0.4 & 1.3 & 11.0 & 58.7 \\
\hline 26 & 17 & $344118-\mathrm{C} 5$ & 6.2 & 43.7 & 0.5 & 0.9 & 0.5 & 0.3 & 0.4 & 0.3 & 0.3 & 8.8 & 61.9 \\
\hline 27 & 18 & 344122-B4 & 0.3 & 0.6 & 0.0 & 0.2 & 0.3 & 0.1 & 0.0 & 0.0 & 0.0 & 5.9 & 7.3 \\
\hline 28 & 18 & $344122-\mathrm{C} 3$ & 0.6 & 1.4 & 0.0 & 0.6 & 0.6 & 0.3 & 0.2 & 0.1 & 0.0 & 5.0 & 8.8 \\
\hline 29 & 19 & $344122-\mathrm{A} 2$ & 0.0 & 0.1 & 0.0 & 0.1 & 0.1 & 0.0 & 0.0 & 0.0 & 0.0 & 7.5 & 7.8 \\
\hline 30 & 19 & 344122-D5 & 0.2 & 0.1 & 0.0 & 0.2 & 0.1 & 0.0 & 0.0 & 0.0 & 0.0 & 7.3 & 7.9 \\
\hline 31 & 20 & 344122-B1 & 51.9 & 15.9 & 11.6 & 23.0 & 5.3 & 2.0 & 3.2 & 1.4 & 1.2 & 10.0 & 125.2 \\
\hline 32 & 20 & $344122-\mathrm{C} 6$ & 72.4 & 22.3 & 15.2 & 33.2 & 7.5 & 2.8 & 4.5 & 2.0 & 1.9 & 10.7 & 172.4 \\
\hline 33 & 21 & 344445-A5 & 0.6 & 17.0 & 0.1 & 0.2 & 0.5 & 0.1 & 0.2 & 0.1 & 0.1 & 7.8 & 26.5 \\
\hline 34 & 21 & 344445-D2 & 1.3 & 21.1 & 0.2 & 0.3 & 0.6 & 0.1 & 0.2 & 0.1 & 0.1 & 5.9 & 29.9 \\
\hline 35 & 22 & 344543-B2 & 25.4 & 9.3 & 8.2 & 11.3 & 4.4 & 1.5 & 2.3 & 1.1 & 0.8 & 6.9 & 71.3 \\
\hline 36 & 22 & $344543-\mathrm{C} 5$ & 31.2 & 11.2 & 9.6 & 13.7 & 5.4 & 1.9 & 2.9 & 1.4 & 1.0 & 9.1 & 87.2 \\
\hline 37 & 23 & 344543-B3 & 162.4 & 229.1 & 15.4 & 29.2 & 19.4 & 12.0 & 2.8 & 0.2 & 0.1 & 7.5 & 477.9 \\
\hline 38 & 23 & 344543-C4 & 145.0 & 206.6 & 13.7 & 26.2 & 17.3 & 10.6 & 2.5 & 0.1 & 0.0 & 6.5 & 428.4 \\
\hline 39 & 24 & 344543-B1 & 0.0 & 2.1 & 0.1 & 0.2 & 0.1 & 0.0 & 0.0 & 0.0 & 0.3 & 8.5 & 11.2 \\
\hline 40 & 24 & 344543-C6 & 0.0 & 3.6 & 0.1 & 0.2 & 0.2 & 0.0 & 0.0 & 0.0 & 0.0 & 6.1 & 10.1 \\
\hline
\end{tabular}

Yielded mass via GC analysis $=(\mathrm{g} / \mathrm{g}$ nonane $) \times 0.050 \mathrm{~g}$ nonane 
Table S13. Products Produced (mg) for Cr Catalysts Evaluated for Ethylene Oligomerization in the High-Throughput Reactor: Ligands 25 - 44

\begin{tabular}{|c|c|c|c|c|c|c|c|c|c|c|c|c|c|}
\hline Entry & Ligand \# & Sample ID & $\begin{array}{c}\text { 1-Octene } \\
(\mathrm{mg})\end{array}$ & $\begin{array}{c}\text { 1-Hexene } \\
(\mathrm{mg})\end{array}$ & $\begin{array}{c}\text { Methylcyclopentane } \\
\text { (mg) }\end{array}$ & $\begin{array}{l}\text { Methylenecyclopentane } \\
\text { (mg) }\end{array}$ & $\begin{array}{l}\mathrm{C} 10 \\
(\mathrm{mg})\end{array}$ & $\begin{array}{l}\mathrm{C} 12 \\
(\mathrm{mg})\end{array}$ & $\begin{array}{l}\mathrm{C} 14 \\
(\mathrm{mg})\end{array}$ & $\begin{array}{l}\mathrm{C} 16 \\
(\mathrm{mg})\end{array}$ & $\begin{array}{l}\mathrm{C} 18 \\
(\mathrm{mg})\end{array}$ & $\begin{array}{c}\mathrm{HDPE} \\
(\mathrm{mg})\end{array}$ & $\begin{array}{l}\text { Total } \\
(\mathrm{mg})\end{array}$ \\
\hline 1 & 25 & $344543-\mathrm{A} 6$ & 0.0 & 31.8 & 0.0 & $\begin{array}{l}0.1 \\
\end{array}$ & 0.3 & 0.1 & 0.1 & 0.1 & 0.2 & 7.7 & 40.4 \\
\hline 2 & 25 & 344543-D1 & 0.0 & 31.0 & 0.1 & 0.1 & 0.3 & 0.1 & 0.2 & 0.2 & 0.3 & 7.5 & 39.6 \\
\hline 3 & 26 & $344108-B 4$ & 1.6 & 118.8 & 0.3 & 1.0 & 1.3 & 0.5 & 1.1 & 0.7 & 1.5 & 13.8 & 140.4 \\
\hline 4 & 26 & 344108-C3 & 1.2 & 69.5 & 0.2 & 0.5 & 0.8 & 0.5 & 0.9 & 0.5 & 0.7 & 18.3 & 92.8 \\
\hline 5 & 27 & $344445-A 3$ & 123.5 & 78.2 & 7.8 & 23.6 & 2.0 & 1.7 & 1.0 & 0.18 & 0.27 & 11.2 & 249.4 \\
\hline 6 & 27 & 344445-D4 & 116.4 & 74.4 & 7.2 & 22.1 & 2.2 & 1.6 & 0.9 & 0.1 & 0.1 & 6.0 & 231.0 \\
\hline 7 & 28 & $344122-B 2$ & 20.1 & 3.7 & 1.9 & 4.3 & 3.3 & 1.4 & 2.7 & 1.9 & 1.6 & 14.0 & 54.8 \\
\hline 8 & 28 & $344122-\mathrm{C} 5$ & 24.5 & 4.4 & 2.4 & 4.8 & 4.2 & 2.0 & 3.5 & 2.5 & 2.2 & 14.1 & 64.5 \\
\hline 9 & 29 & $344122-\mathrm{A} 6$ & 1.3 & 2.8 & 0.1 & 0.1 & 0.7 & 0.3 & 0.4 & 0.1 & 0.2 & 39.6 & 45.4 \\
\hline 10 & 29 & 344122-D1 & 0.9 & 1.9 & 0.0 & 0.1 & 0.4 & 0.1 & 0.2 & 0.1 & 0.1 & 28.3 & 32.1 \\
\hline 11 & 30 & 344108-A6 & 5.3 & 2.8 & 0.4 & 1.1 & 0.5 & 0.0 & 1.7 & 0.2 & 0.6 & 10.4 & 23.0 \\
\hline 12 & 30 & 344108-D1 & 4.0 & 2.0 & 0.3 & 0.5 & 0.2 & 0.0 & 1.1 & 0.1 & 0.3 & 11.1 & 19.5 \\
\hline 13 & 31 & 344108-A3 & 128.0 & 64.6 & 8.2 & 12.1 & 3.2 & 2.5 & 2.6 & 1.4 & 1.1 & 13.7 & 237.2 \\
\hline 14 & 31 & 344108-D4 & 165.0 & 84.9 & 10.4 & 15.5 & 4.8 & 3.9 & 4.7 & 2.5 & 3.2 & 32.4 & 327.2 \\
\hline 15 & 32 & $344108-\mathrm{A} 2$ & 380.9 & 250.1 & 12.1 & 16.9 & 10.8 & 15.3 & 6.8 & 1.8 & 1.1 & 13.4 & 709.1 \\
\hline 16 & 32 & 344108-D5 & 333.5 & 218.7 & 10.6 & 14.8 & 9.1 & 13.0 & 6.8 & 1.3 & 2.7 & 17.4 & 627.8 \\
\hline 17 & 33 & 344114-A5 & 0.3 & 11.4 & 0.0 & 0.1 & 0.3 & 0.3 & 0.5 & 0.4 & 1.2 & 47.8 & 62.3 \\
\hline 18 & 33 & 344114-D2 & 0.4 & 17.4 & 0.0 & 0.1 & 0.5 & 0.5 & 0.7 & 0.6 & 0.9 & 72.9 & 93.8 \\
\hline 19 & 34 & 344114-A3 & 0.5 & 0.6 & 0.0 & 0.1 & 0.6 & 0.6 & 0.7 & 0.6 & 0.7 & 26.7 & 31.0 \\
\hline 20 & 34 & 344114-D4 & 0.8 & 0.9 & 0.0 & 0.1 & 1.1 & 1.1 & 1.3 & 1.2 & 1.7 & 46.7 & 54.9 \\
\hline 21 & 35 & 344114-B3 & 0.2 & 9.7 & 0.0 & 0.1 & 0.2 & 0.2 & 0.3 & 0.2 & 0.4 & 33.2 & 44.5 \\
\hline 22 & 35 & 344114-C4 & 0.3 & 9.0 & 0.0 & 0.1 & 0.2 & 0.2 & 0.3 & 0.2 & 4.8 & 32.4 & 47.4 \\
\hline 23 & 36 & 344114-A6 & 0.2 & 2.3 & 0.0 & 0.1 & 0.2 & 0.2 & 0.3 & 0.2 & 0.6 & 15.9 & 19.8 \\
\hline 24 & 36 & 344114-D1 & 0.2 & 3.6 & 0.0 & 0.1 & 0.2 & 0.2 & 0.3 & 0.2 & 0.2 & 25.9 & 30.8 \\
\hline 25 & 37 & 344114-B1 & 0.2 & 0.4 & 0.0 & 0.1 & 0.1 & 0.0 & 0.0 & 0.0 & 0.5 & 10.3 & 11.4 \\
\hline 26 & 37 & 344114-C6 & 0.2 & 0.4 & 0.0 & 0.1 & 0.1 & 0.0 & 0.0 & 0.0 & 0.2 & 3.6 & 4.5 \\
\hline 27 & 38 & 344114-A2 & 0.3 & 0.4 & 0.0 & 0.1 & 0.2 & 0.1 & 0.1 & 0.4 & 0.2 & 11.9 & 13.6 \\
\hline 28 & 38 & 344114-D5 & 0.3 & 0.3 & 0.0 & 0.1 & 0.1 & 0.0 & 0.0 & 0.0 & 0.2 & 9.1 & 10.1 \\
\hline 29 & 39 & 344118-A4 & 0.3 & 0.4 & 0.0 & 0.4 & 0.3 & 0.3 & 0.7 & 0.3 & 0.3 & 8.8 & 11.8 \\
\hline 30 & 39 & 344118-D3 & 0.4 & 0.6 & 0.0 & 0.1 & 0.5 & 0.4 & 0.5 & 0.4 & 1.3 & 16.8 & 20.9 \\
\hline 31 & 40 & 344118-A3 & 0.0 & 0.2 & 0.0 & 0.2 & 0.2 & 0.0 & 0.0 & 0.0 & 0.2 & 14.4 & 15.1 \\
\hline 32 & 40 & 344118-D4 & 0.2 & 0.3 & 0.0 & 0.1 & 0.1 & 0.0 & 0.0 & 0.0 & 0.0 & 20.3 & 21.0 \\
\hline 33 & 41 & 344118-B5 & 23.0 & 5.6 & 1.5 & 3.4 & 2.2 & 1.7 & 3.3 & 2.1 & 1.9 & 38.0 & 82.6 \\
\hline 34 & 41 & 344118-C2 & 20.9 & 4.9 & 1.5 & 2.3 & 1.8 & 1.3 & 1.9 & 1.5 & 1.5 & 41.5 & 79.0 \\
\hline 35 & 42 & $344118-\mathrm{A} 2$ & 0.2 & 0.6 & 0.0 & 0.1 & 0.3 & 0.3 & 0.5 & 0.9 & 0.7 & 82.7 & 86.2 \\
\hline 36 & 42 & 344118-D5 & 0.3 & 0.8 & 0.0 & 0.1 & 0.3 & 0.3 & 0.5 & 0.5 & 0.4 & 97.5 & 100.6 \\
\hline 37 & 43 & 344118-B4 & 0.1 & 6.8 & 0.0 & 0.1 & 0.1 & 0.0 & 0.0 & 0.0 & 0.0 & 5.0 & 12.1 \\
\hline 38 & 43 & 344118-C3 & 0.0 & 7.7 & 0.0 & 0.1 & 0.1 & 0.0 & 0.0 & 0.0 & 0.7 & 6.0 & 14.6 \\
\hline 39 & 44 & 344118-B1 & 0.5 & 1.2 & 0.0 & 0.2 & 0.5 & 0.5 & 0.6 & 0.5 & 0.4 & 9.8 & 14.1 \\
\hline 40 & 44 & 344118-C6 & 0.6 & 1.6 & 0.0 & 0.2 & 0.8 & 0.7 & 0.7 & 0.6 & 0.6 & 14.0 & 19.8 \\
\hline
\end{tabular}

Yielded mass via $\mathrm{GC}$ analysis $=(\mathrm{g} / \mathrm{g}$ nonane $) \times 0.050 \mathrm{~g}$ nonane 
Table S14. Products Produced (mg) for Cr Catalysts Evaluated for Ethylene Oligomerization in the High-Throughput Reactor: Ligands 45 - 57

\begin{tabular}{|c|c|c|c|c|c|c|c|c|c|c|c|c|c|}
\hline Entry & Ligand \# & Sample ID & $\begin{array}{c}\text { 1-Octene } \\
(\mathrm{mg})\end{array}$ & $\begin{array}{c}\text { 1-Hexene } \\
(\mathrm{mg})\end{array}$ & $\begin{array}{c}\text { Methylcyclopentane } \\
(\mathrm{mg})\end{array}$ & $\begin{array}{l}\text { Methylenecyclopentane } \\
(\mathrm{mg})\end{array}$ & $\begin{array}{l}\mathrm{C} 10 \\
(\mathrm{mg})\end{array}$ & $\begin{array}{l}\mathrm{C} 12 \\
(\mathrm{mg})\end{array}$ & $\begin{array}{l}\mathrm{C} 14 \\
(\mathrm{mg})\end{array}$ & $\begin{array}{l}\mathrm{C} 16 \\
\text { (mg) }\end{array}$ & $\begin{array}{l}\mathrm{C} 18 \\
\text { (mg) }\end{array}$ & $\begin{array}{c}\mathrm{HDPE} \\
(\mathrm{mg})\end{array}$ & $\begin{array}{l}\text { Total } \\
(\mathrm{mg})\end{array}$ \\
\hline 1 & 45 & $344126-\mathrm{A} 2$ & 0.0 & 0.2 & 0.0 & 0.1 & 0.1 & 0.0 & 0.0 & 0.0 & 0.2 & 4.6 & 5.1 \\
\hline 2 & 45 & 344126-D5 & 0.1 & 0.2 & 0.0 & 0.1 & 0.1 & 0.0 & 0.0 & 0.0 & 0.0 & 6.4 & 6.8 \\
\hline 3 & 46 & $344126-A 3$ & 0.0 & 0.4 & 0.0 & 0.2 & 0.1 & 0.0 & 0.0 & 0.0 & 0.0 & 4.2 & 4.8 \\
\hline 4 & 46 & 344126-D4 & 0.0 & 0.6 & 0.0 & 0.4 & 0.1 & 0.0 & 0.0 & 0.0 & 0.0 & 9.3 & 10.4 \\
\hline 5 & 47 & $344126-\mathrm{A} 6$ & 1.1 & 1.7 & 0.0 & 0.3 & 1.1 & 1.0 & 1.0 & 0.8 & 0.7 & 11.1 & 18.8 \\
\hline 6 & 47 & 344126-D1 & 1.5 & 2.0 & 0.0 & 0.4 & 1.6 & 1.4 & 1.4 & 1.2 & 1.0 & 11.6 & 21.9 \\
\hline 7 & 48 & 344118-A6 & 3.2 & 3.4 & 0.0 & 0.2 & 3.8 & 3.2 & 3.1 & 2.4 & 2.0 & 14.5 & 35.7 \\
\hline 8 & 48 & 344118-D1 & 6.6 & 6.8 & 0.0 & 0.3 & 7.7 & 6.8 & 6.2 & 5.0 & 4.1 & 29.1 & 72.6 \\
\hline 9 & 49 & 344118-A5 & 0.0 & 0.2 & 0.0 & 0.1 & 0.1 & 0.0 & 0.0 & 0.0 & 0.0 & 8.2 & 8.5 \\
\hline 10 & 49 & 344118-D2 & 0.2 & 0.2 & 0.0 & 0.1 & 0.1 & 0.0 & 0.0 & 0.1 & 0.1 & 9.5 & 10.2 \\
\hline 11 & 50 & 344118-B3 & 0.1 & 0.1 & 0.0 & 0.1 & 0.1 & 0.0 & 0.0 & 0.0 & 0.0 & 8.9 & 9.2 \\
\hline 12 & 50 & 344118-C4 & 0.2 & 0.3 & 0.0 & 0.1 & 0.1 & 0.0 & 0.0 & 0.0 & 0.8 & 9.2 & 10.6 \\
\hline 13 & 51 & $344122-B 3$ & 0.9 & 2.2 & 0.0 & 0.2 & 1.1 & 0.9 & 1.0 & 0.8 & 0.7 & 8.1 & 15.7 \\
\hline 14 & 51 & $344122-\mathrm{C} 4$ & 1.0 & 2.6 & 0.0 & 0.2 & 1.2 & 1.0 & 1.0 & 0.8 & 0.7 & 9.0 & 17.4 \\
\hline 15 & 52 & 344122-B5 & 0.0 & 0.7 & 0.0 & 1.4 & 0.1 & 0.0 & 0.0 & 0.0 & 0.0 & 3.8 & 6.0 \\
\hline 16 & 52 & $344122-\mathrm{C} 2$ & 0.3 & 0.6 & 0.0 & 1.2 & 0.2 & 0.1 & 0.1 & 0.0 & 0.0 & 6.6 & 9.0 \\
\hline 17 & 53 & $344122-\mathrm{A} 4$ & 0.0 & 0.7 & 0.0 & 1.3 & 0.3 & 0.1 & 0.1 & 0.0 & 0.0 & 6.5 & 8.9 \\
\hline 18 & 53 & 344122-D3 & 0.7 & 1.5 & 0.0 & 0.2 & 0.6 & 0.3 & 0.1 & 0.1 & 0.0 & 7.1 & 10.5 \\
\hline 19 & 54 & $344122-\mathrm{A} 3$ & 0.1 & 0.1 & 0.0 & 0.1 & 0.1 & 0.0 & 0.0 & 0.0 & 0.0 & 5.1 & 5.4 \\
\hline 20 & 54 & 344122-D4 & 0.0 & 0.1 & 0.0 & 0.1 & 0.1 & 0.0 & 0.0 & 0.0 & 0.0 & 4.4 & 4.6 \\
\hline 21 & 55 & 344126-B1 & 0.0 & 0.1 & 0.0 & 0.3 & 0.1 & 0.0 & 0.4 & 0.0 & 0.0 & 4.6 & 5.5 \\
\hline 22 & 55 & 344126-C6 & 0.1 & 0.2 & 0.0 & 0.1 & 0.1 & 0.0 & 0.0 & 0.0 & 0.0 & 10.6 & 11.0 \\
\hline 23 & 56 & 344126-A5 & 0.0 & 0.2 & 0.0 & 0.0 & 0.1 & 0.0 & 0.0 & 0.0 & 0.0 & 4.5 & 4.8 \\
\hline 24 & 56 & 344126-D2 & 0.0 & 0.1 & 0.0 & 0.0 & 0.1 & 0.0 & 0.7 & 0.0 & 0.0 & 7.9 & 8.8 \\
\hline 25 & 57 & 344445-A6 & 0.0 & 19.8 & 0.0 & 0.1 & 0.5 & 0.0 & 0.1 & 0.0 & 0.1 & 7.1 & 27.6 \\
\hline 26 & 57 & 344445-D1 & 0.0 & 17.9 & 0.0 & 0.1 & 0.5 & 0.0 & 0.2 & 0.1 & 0.1 & 9.6 & 28.4 \\
\hline
\end{tabular}

Yielded mass via $\mathrm{GC}$ analysis $=(\mathrm{g} / \mathrm{g}$ nonane $) \times 0.050 \mathrm{~g}$ nonane 


\section{Example of Activity and Selectivity Calculations: Ligand 1 and Ligand 27}

Table S15. Oligomerization Conditions

\begin{tabular}{|c|c|c|c|c|c|c|c|}
\hline Catalyst & $\begin{array}{c}\text { Catalyst } \\
(\mu \mathrm{mol})\end{array}$ & $\begin{array}{c}\text { Temperature } \\
\left({ }^{\circ} \mathrm{C}\right)\end{array}$ & $\begin{array}{c}\text { Pressure } \\
(\mathrm{psi})\end{array}$ & $\begin{array}{c}\text { Run time } \\
(\mathrm{min})\end{array}$ & $\begin{array}{c}\text { Nonane } \\
(\mathrm{mg})\end{array}$ & $\begin{array}{c}\text { MMAO-3A:Cr } \\
\text { ratio }\end{array}$ & $\begin{array}{c}\text { MMAO- } \\
\text { 3A }(\mu \mathrm{mol})\end{array}$ \\
\hline $\mathbf{1}$ & 0.05 & 60 & 500 & 30 & 50 & $1000: 1$ & 50 \\
\hline $\mathbf{2 7}$ & 0.05 & 60 & 500 & 30 & 50 & $1000: 1$ & 50 \\
\hline
\end{tabular}

Table S16. GC raw data

\begin{tabular}{|c|c|c|c|c|}
\hline Catalyst & $\begin{array}{c}\text { 1-Octene } \\
(\mathrm{g} / \mathrm{g} \text { nonane }) \\
(6.9 \mathrm{~min})\end{array}$ & $\begin{array}{c}\text { 1-Hexene } \\
(\mathrm{g} / \mathrm{g} \text { nonane }) \\
(2.8 \mathrm{~min})\end{array}$ & $\begin{array}{c}\text { Methylcyclopentane } \\
(\mathrm{g} / \mathrm{g} \text { nonane }) \\
(3.3 \mathrm{~min})\end{array}$ & $\begin{array}{c}\text { Methylenecyclopentane } \\
(\mathrm{g} / \mathrm{g} \text { nonane }) \\
(3.6 \mathrm{~min})\end{array}$ \\
\hline $\mathbf{1}$ & 7.482 & 3.033 & 0.2450 & 0.2770 \\
\hline $\mathbf{2 7}$ & 2.470 & 1.564 & 0.1550 & 0.4720 \\
\hline
\end{tabular}

\begin{tabular}{|c|c|c|c|c|c|}
\hline Catalyst & $\begin{array}{c}\text { Decenes } \\
(\mathrm{g} / \mathrm{g} \text { nonane }) \\
(10.0-11.0 \mathrm{~min})\end{array}$ & $\begin{array}{c}\text { Dodecenes } \\
(\mathrm{g} / \mathrm{g} \text { nonane }) \\
(11.3-11.8 \mathrm{~min})\end{array}$ & $\begin{array}{c}\text { Tetradecenes } \\
(\mathrm{g} / \mathrm{g} \text { nonane }) \\
(11.8-12.7 \mathrm{~min})\end{array}$ & $\begin{array}{c}\text { Hexadecenes } \\
(\mathrm{g} / \mathrm{g} \text { nonane }) \\
(13.0-13.5 \mathrm{~min})\end{array}$ & $\begin{array}{c}\text { Octadecenes } \\
(\mathrm{g} / \mathrm{g} \text { nonane }) \\
(13.7-14.2 \mathrm{~min})\end{array}$ \\
\hline $\mathbf{1}$ & 0.2147 & 0.2970 & 0.1947 & 0.0393 & 0.0346 \\
\hline $\mathbf{2 7}$ & 0.0394 & 0.0333 & 0.0202 & 0.0038 & 0.0055 \\
\hline
\end{tabular}

* GC retention times

Table S17. Yielded Mass via GC Analysis $=(\mathrm{g} / \mathrm{g}$ nonane $) \times 0.050 \mathrm{~g}$ nonane

\begin{tabular}{|c|c|c|c|c|c|}
\hline Catalyst & $\begin{array}{c}\text { 1-Octene } \\
(\mathrm{g})\end{array}$ & $\begin{array}{c}\text { 1-Hexene } \\
(\mathrm{g})\end{array}$ & $\begin{array}{c}\text { Methylcyclopentane } \\
(\mathrm{g})\end{array}$ & $\begin{array}{c}\text { Methylenecyclopentane } \\
(\mathrm{g})\end{array}$ & $\begin{array}{c}\text { Polymer* } \\
(\mathrm{g})\end{array}$ \\
\hline $\mathbf{1}$ & 0.3741 & 0.1517 & 0.0123 & 0.0139 & 0.0152 \\
\hline $\mathbf{2 7}$ & 0.1235 & 0.0782 & 0.0078 & 0.0236 & 0.0112 \\
\hline
\end{tabular}

\begin{tabular}{|c|c|c|c|c|c|c|}
\hline Catalyst & $\begin{array}{c}\text { Decenes } \\
(\mathrm{g})\end{array}$ & $\begin{array}{c}\text { Dodecenes } \\
(\mathrm{g})\end{array}$ & $\begin{array}{c}\text { Tetradecenes } \\
(\mathrm{g})\end{array}$ & $\begin{array}{c}\text { Hexadecenes } \\
(\mathrm{g})\end{array}$ & $\begin{array}{c}\text { Octadecenes } \\
(\mathrm{g})\end{array}$ & $\begin{array}{c}\text { Total } \\
(\mathbf{g})\end{array}$ \\
\hline $\mathbf{1}$ & 0.0107 & 0.0148 & 0.0097 & 0.0020 & 0.0017 & $\mathbf{0 . 6 0 6 1}$ \\
\hline $\mathbf{2 7}$ & 0.0020 & 0.0017 & 0.0010 & 0.0019 & 0.0003 & $\mathbf{0 . 2 5 1 2}$ \\
\hline
\end{tabular}

*Polymer mass obtained by gravimetric analysis

Table S18. Activity $(\mathrm{g} / \mathrm{g} \mathrm{Cr} \mathrm{hr})=\frac{\mathrm{g} \text { product }}{\left(\left(\frac{\mu \mathrm{mol} \mathrm{Cr}}{1,000,000}\right) \times 52\right)(\mathrm{hr})}$

\begin{tabular}{|c|c|c|c|c|c|}
\hline Catalyst & $\begin{array}{c}\text { 1-Octene } \\
(\mathrm{g} / \mathrm{g} \mathrm{Cr} \mathrm{hr})\end{array}$ & $\begin{array}{c}\text { 1-Hexene } \\
(\mathrm{g} / \mathrm{g} \mathrm{Cr} \mathrm{hr})\end{array}$ & $\begin{array}{c}\text { Methylcyclopentane } \\
(\mathrm{g} / \mathrm{g} \mathrm{Cr} \mathrm{hr})\end{array}$ & $\begin{array}{c}\text { Methylenecyclopentane } \\
(\mathrm{g} / \mathrm{g} \mathrm{Cr} \mathrm{hr})\end{array}$ & $\begin{array}{c}\text { Polymer } \\
(\mathrm{g} / \mathrm{g} \mathrm{Cr} \mathrm{hr})\end{array}$ \\
\hline $\mathbf{1}$ & 287,800 & 116,700 & 9,423 & 10,650 & 11,690 \\
\hline $\mathbf{2 7}$ & 95,000 & 60,150 & 5,962 & 18,154 & 8,615 \\
\hline
\end{tabular}

\begin{tabular}{|c|c|c|c|c|c|c|}
\hline Catalyst & $\begin{array}{c}\text { Decenes } \\
(\mathrm{g} / \mathrm{g} \mathrm{Cr} \mathrm{hr})\end{array}$ & $\begin{array}{c}\text { Dodecenes } \\
(\mathrm{g} / \mathrm{g} \mathrm{Cr} \mathrm{hr})\end{array}$ & $\begin{array}{c}\text { Tetradecenes } \\
(\mathrm{g} / \mathrm{g} \mathrm{Cr} \mathrm{hr})\end{array}$ & $\begin{array}{c}\text { Hexadecenes } \\
(\mathrm{g} / \mathrm{g} \mathrm{Cr} \mathrm{hr})\end{array}$ & $\begin{array}{c}\text { Octadecenes } \\
(\mathrm{g} / \mathrm{g} \mathrm{Cr} \mathrm{hr})\end{array}$ & $\begin{array}{c}\text { Total Activity } \\
(\mathrm{g} / \mathbf{g ~ C r} \mathbf{~ h r})\end{array}$ \\
\hline $\mathbf{1}$ & 8,259 & 11,420 & 7,490 & 1,510 & 1,330 & $\mathbf{4 6 6 , 2 0 0}$ \\
\hline $\mathbf{2 7}$ & 1,516 & 1,280 & 778 & 145 & 210 & $\mathbf{1 9 1 , 8 0 0}$ \\
\hline
\end{tabular}


Table S19. Selectivity $($ wt $\%)=\left(\frac{\text { g product }}{\text { total g product }}\right) \times 100$

\begin{tabular}{|c|c|c|c|c|c|}
\hline Catalyst & $\begin{array}{c}\text { 1-Octene } \\
(\mathrm{wt} \%)\end{array}$ & $\begin{array}{c}\text { 1-Hexene } \\
(\mathrm{wt} \%)\end{array}$ & $\begin{array}{c}\text { Methylcyclopentane } \\
\text { (wt\%) }\end{array}$ & $\begin{array}{c}\text { Methylenecyclopentane } \\
\text { (wt\%) }\end{array}$ & $\begin{array}{c}\text { Polymer } \\
\text { (wt\%) }\end{array}$ \\
\hline $\mathbf{1}$ & 61.7 & 25.0 & 2.0 & 2.3 & 2.5 \\
\hline $\mathbf{2 7}$ & 49.5 & 31.4 & 3.1 & 9.5 & 4.5 \\
\hline
\end{tabular}

\begin{tabular}{|c|c|c|c|c|c|c|}
\hline Catalyst & $\begin{array}{c}\text { Decenes } \\
(\mathrm{wt} \%)\end{array}$ & $\begin{array}{c}\text { Dodecenes } \\
(\mathrm{wt} \%)\end{array}$ & $\begin{array}{c}\text { Tetradecenes } \\
(\mathrm{wt} \%)\end{array}$ & $\begin{array}{c}\text { Hexadecenes } \\
(\mathrm{wt} \%)\end{array}$ & $\begin{array}{c}\text { Octadecenes } \\
(\mathrm{wt} \%)\end{array}$ & $\begin{array}{c}\text { Total Selectivity } \\
(\mathrm{wt} \%)\end{array}$ \\
\hline $\mathbf{1}$ & 1.8 & 2.4 & 1.6 & 0.3 & 0.3 & 100.0 \\
\hline $\mathbf{2 7}$ & 0.8 & 0.7 & 0.4 & 0.1 & 0.1 & 100.0 \\
\hline
\end{tabular}

Ligands were duplicated in each library. The selectivity and activities obtained from each replicate were averaged together. 